\title{
Density Functional Theory Evaluation of a Photoinduced Intramolecular Aryl Ether Rearrangement
}

Péter Pál Fehér*

Research Centre for Natural Sciences, Magyar tudósok körútja 2, H-1117 Budapest, Hungary

*E-mail:feher.peter@ttk.hu 


\section{List of contents}

Fig. S1. The most stable conformations of the protonated and neutral 1a. Page S3

Fig. S2-3. Natural transition orbitals of the (protonated) 1a. Page S4

Fig. S4. The structure of the epoxide intermediates. Page S5

Scheme S1. Inferior reaction steps for the mechanism of 1c shown in Fig. 5. Page S5

Fig. S5. The calculated absorption spectra of the three salicylaldehyde ethers. $\quad$ Page S6

Figs. S6-7. The calculated absorption spectra of the $\mathrm{Me}$ and $\mathrm{NO}_{2}$ substituted 1a+. $\quad$ Page $\mathrm{S} 7$

Fig. S8. NTOs of the first excited state of the molecules shown in Fig. $7 . \quad$ Page S8

Figs. S9-11. The calculated absorption spectra of the modified salicylaldehyde ethers. Pages S9-11

Fig. S12. The ipso attack step for 1a in the S1 state. Page S12

$\begin{array}{lr}\text { Coordinates and energies of the computed structures } & \text { Pages S13-19 }\end{array}$ 

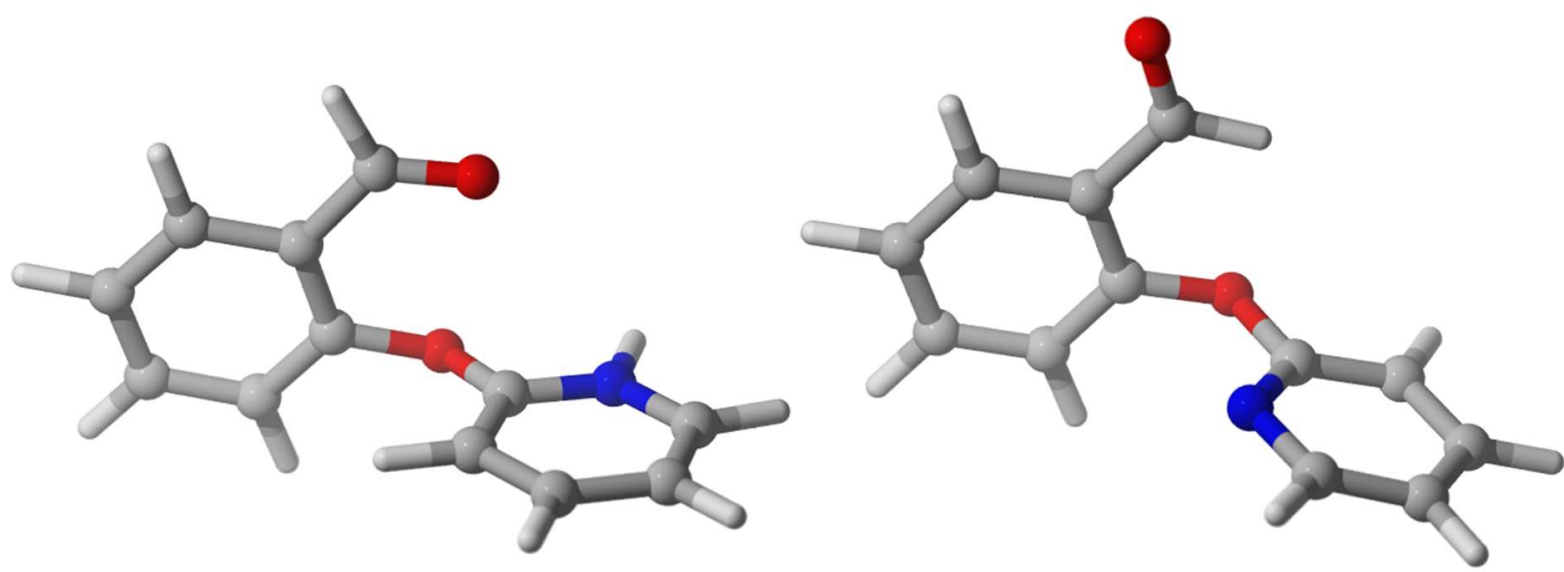

Figure S1. The most stable conformations of the protonated (left) and neutral (right) forms of the 2(pyridin-2-yloxy)benzaldehyde (1a) molecule. 

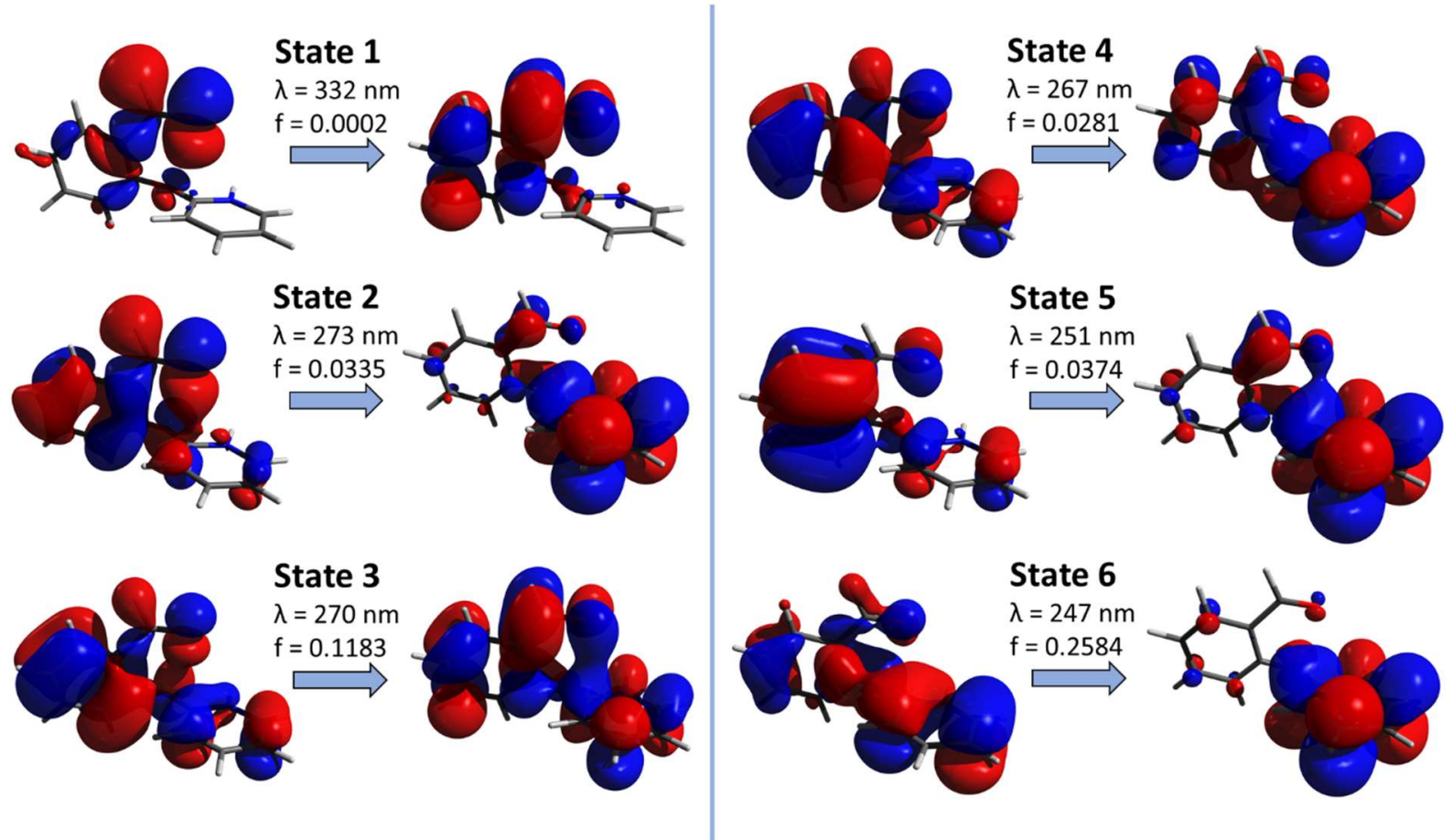

Figure S2. The first six natural transition orbitals (NTOs) of $\mathbf{1 a +}$
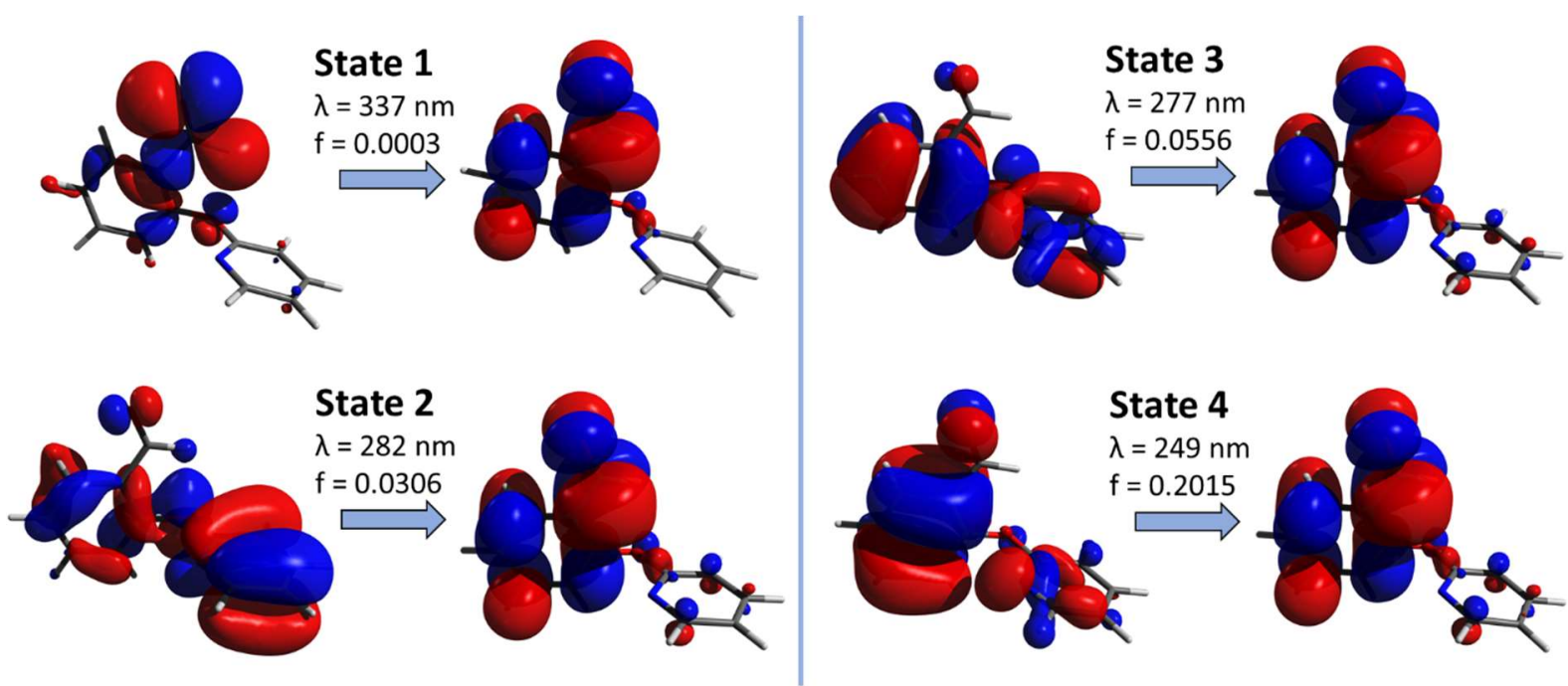

Figure S3. The first six natural transition orbitals (NTOs) of $\mathbf{1 a .}$ 


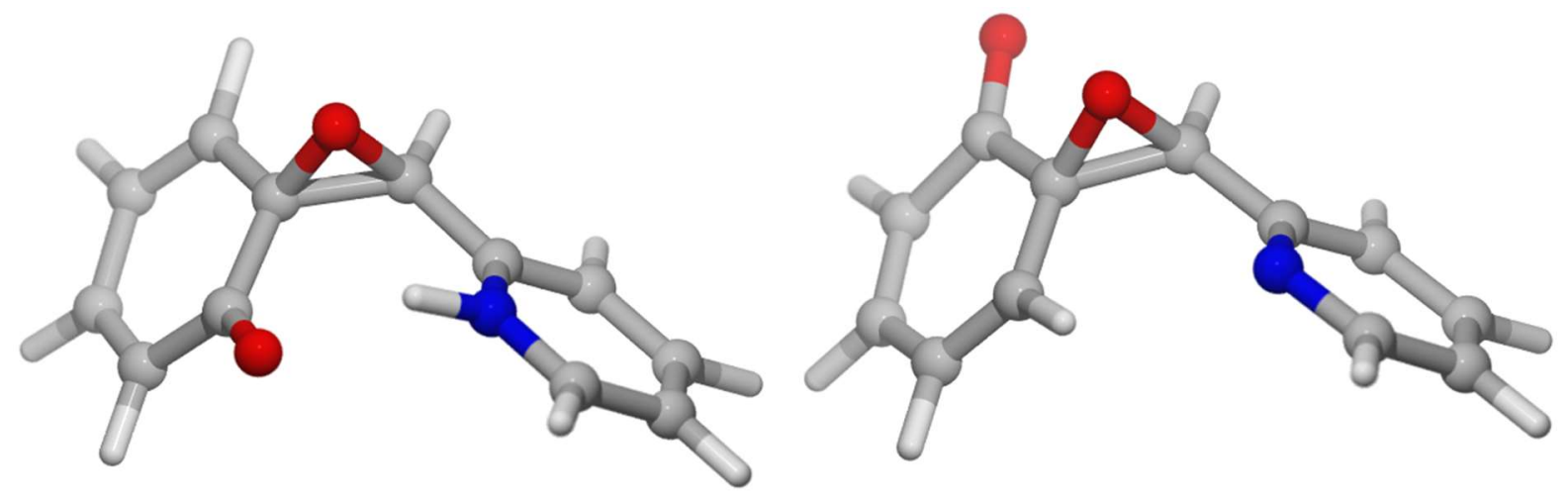

Figure S4. The structure of the epoxide intermediates obtained after the relaxation of $\mathbf{3 a}+(\mathrm{left})$ and $\mathbf{3 a}$ (right) to the ground state.

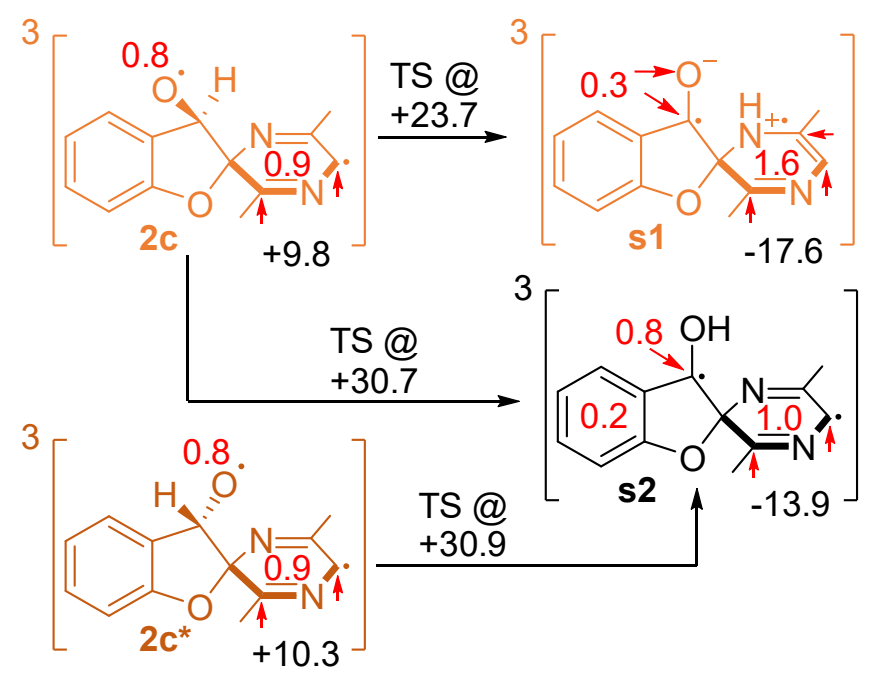

Scheme S1. Inferior reaction steps for the pyrazine ether mechanism shown in Fig. 5. 


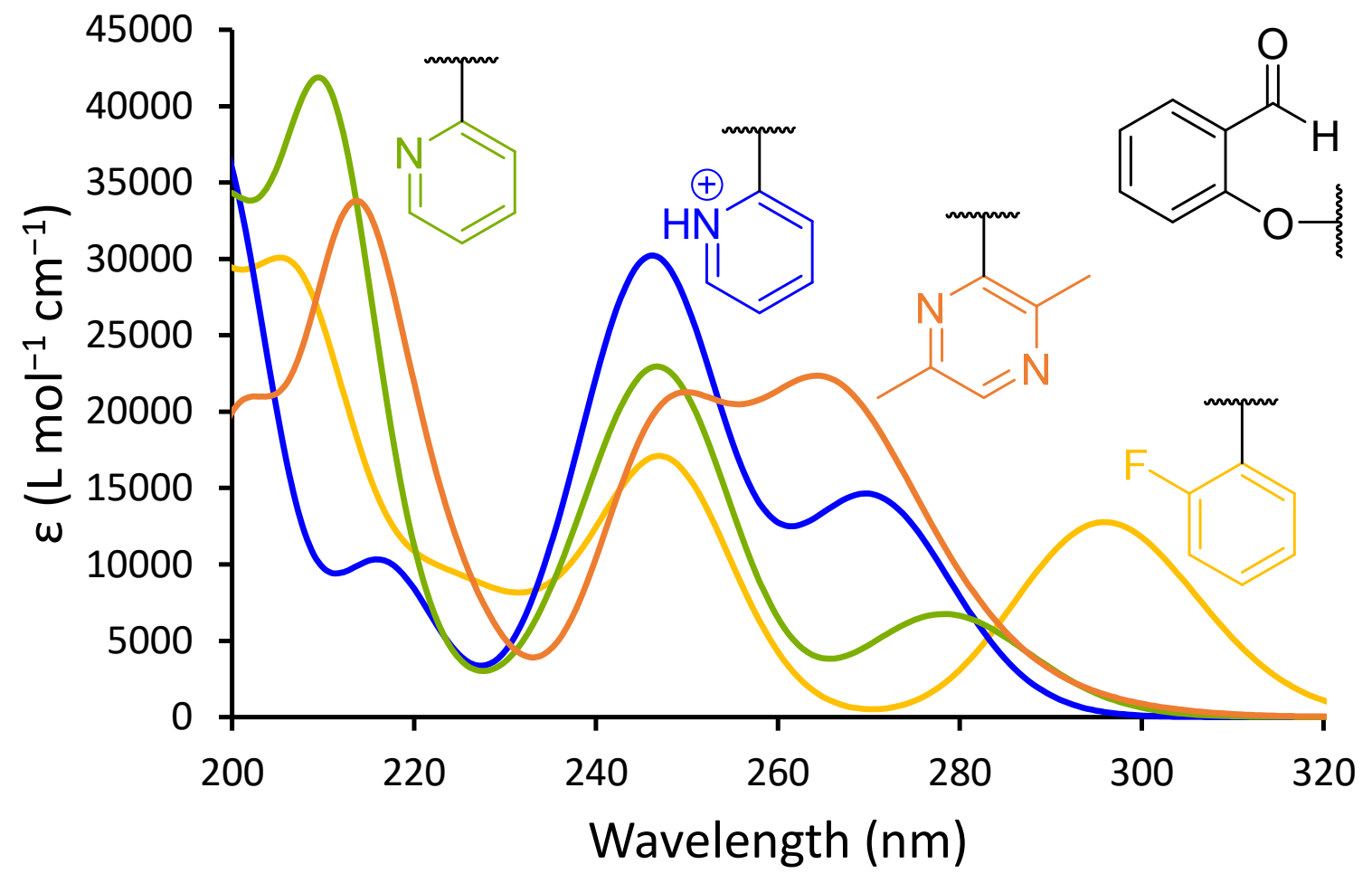

Figure S5. The calculated absorption spectra of the salicylaldehyde ethers investigated in the text. 


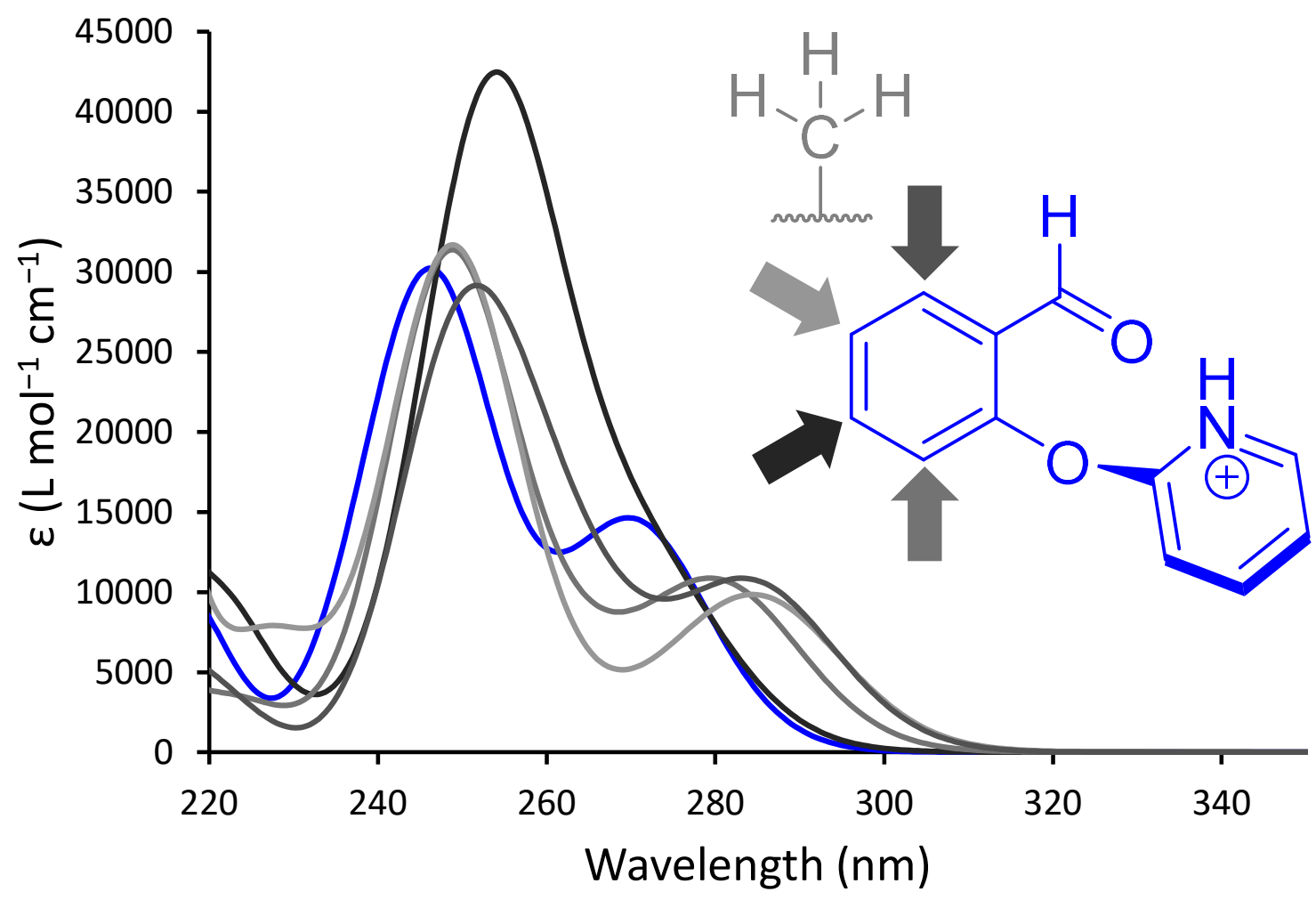

Figure S6. The calculated absorption spectra of the methyl substituted 1a+ derivatives.

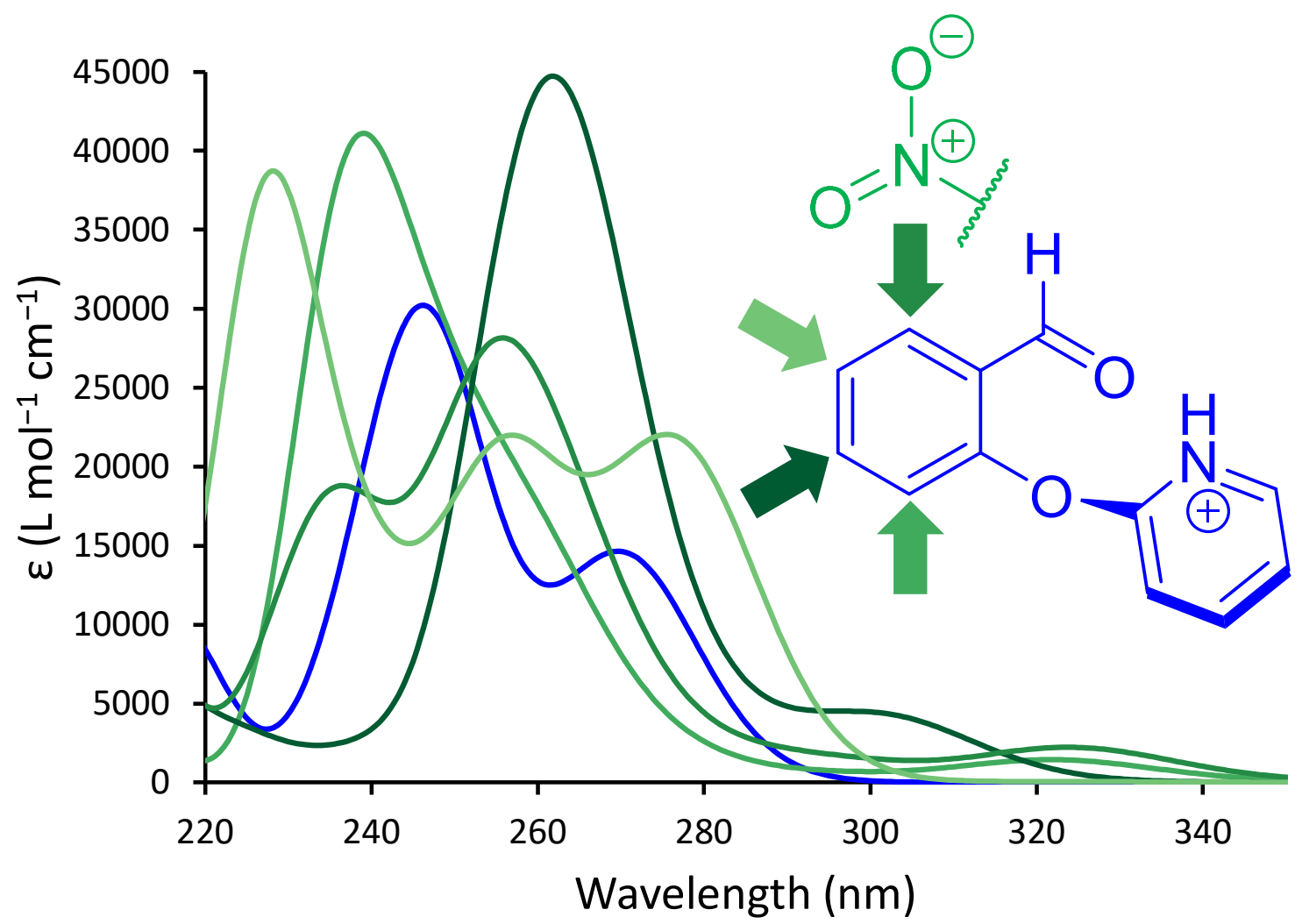

Figure S7. The calculated absorption spectra of the nitro substituted 1a+derivatives. 

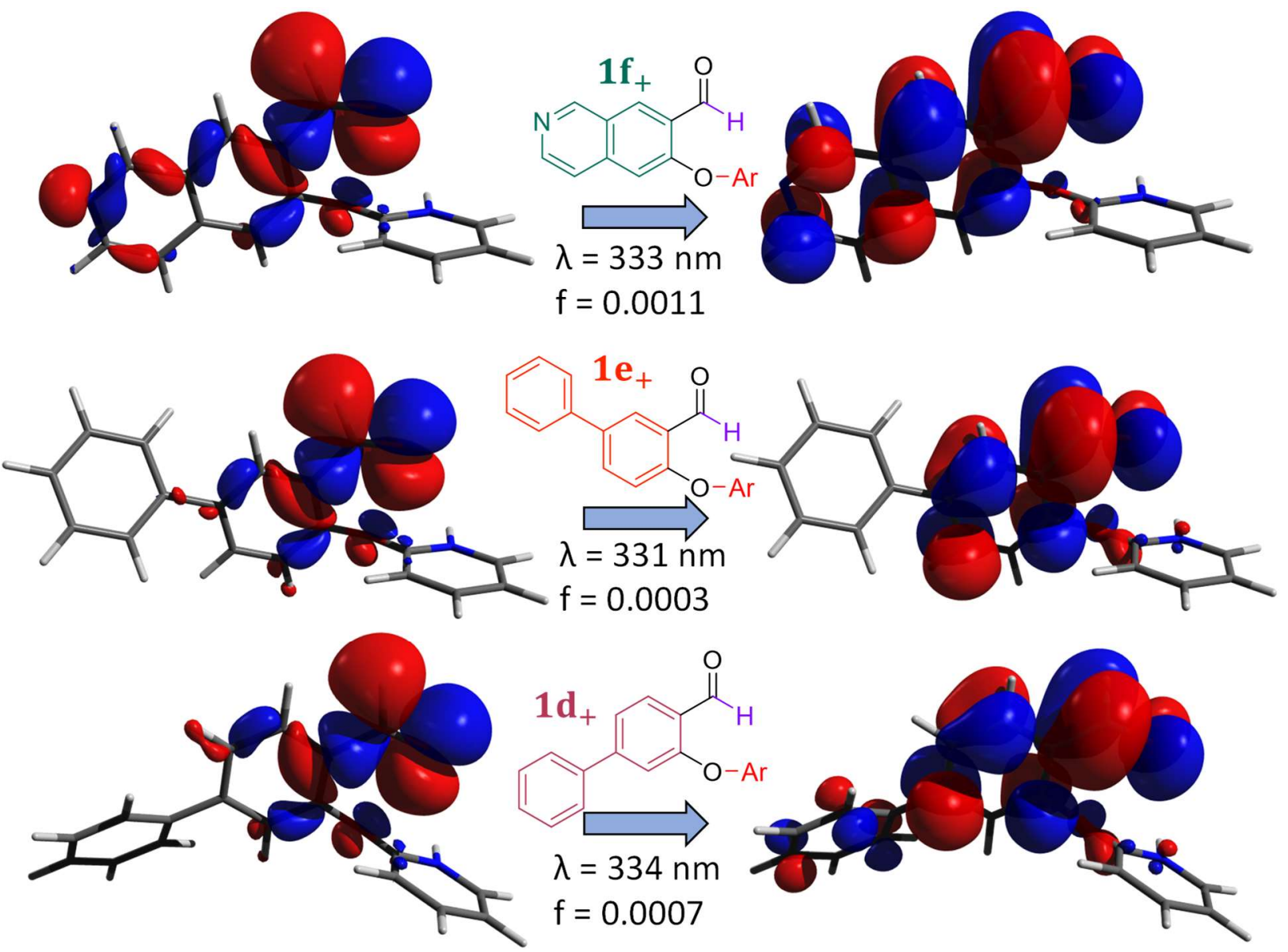

Figure S8. NTOs of the first excited state of the molecules shown in Fig. 7. 


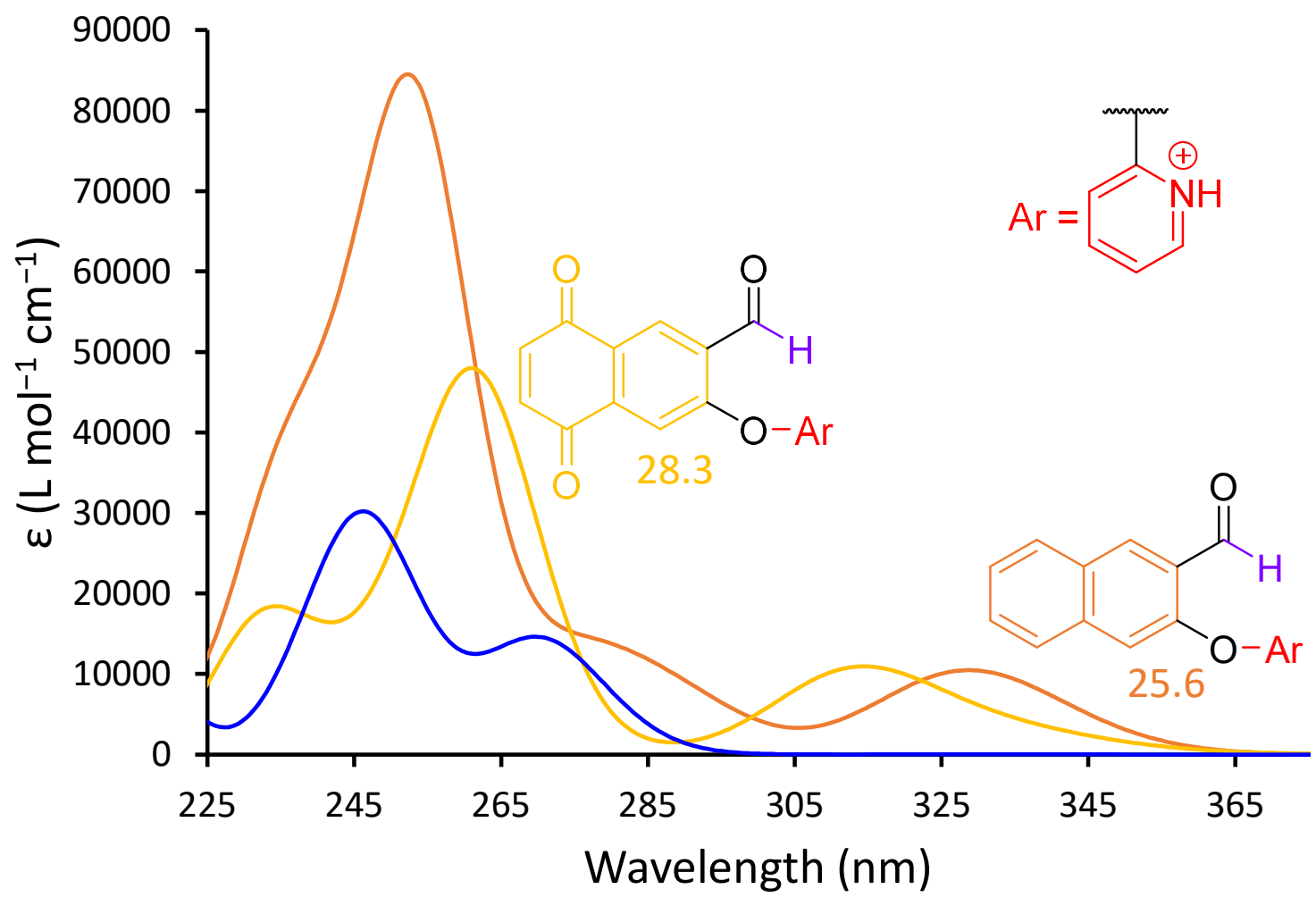

Figure S9. The calculated absorption spectra of the modified (quinone, naphthalene) salicylaldehyde ethers. The blue spectrum corresponds to the absorption of $\mathbf{1} \mathbf{a}+$. The numbers below the structures indicate the barrier of the ipso addition. 


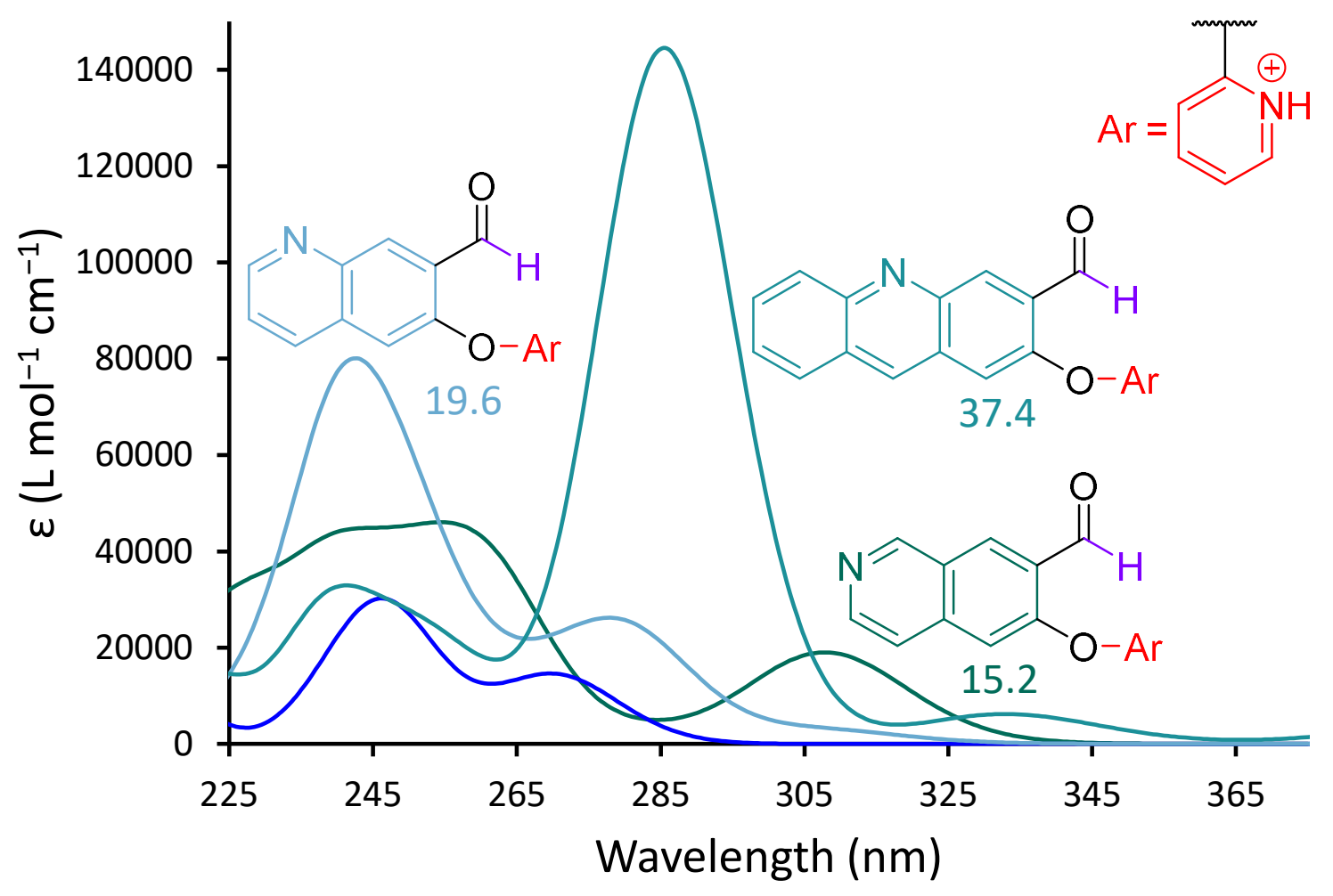

Figure S10. The calculated absorption spectra of the modified (1-quinoline, 2-quinoline, acridine) salicylaldehyde ethers. The blue spectrum corresponds to the absorption of $\mathbf{1 a}+$. The numbers below the structures indicate the barrier of the ipso addition. 


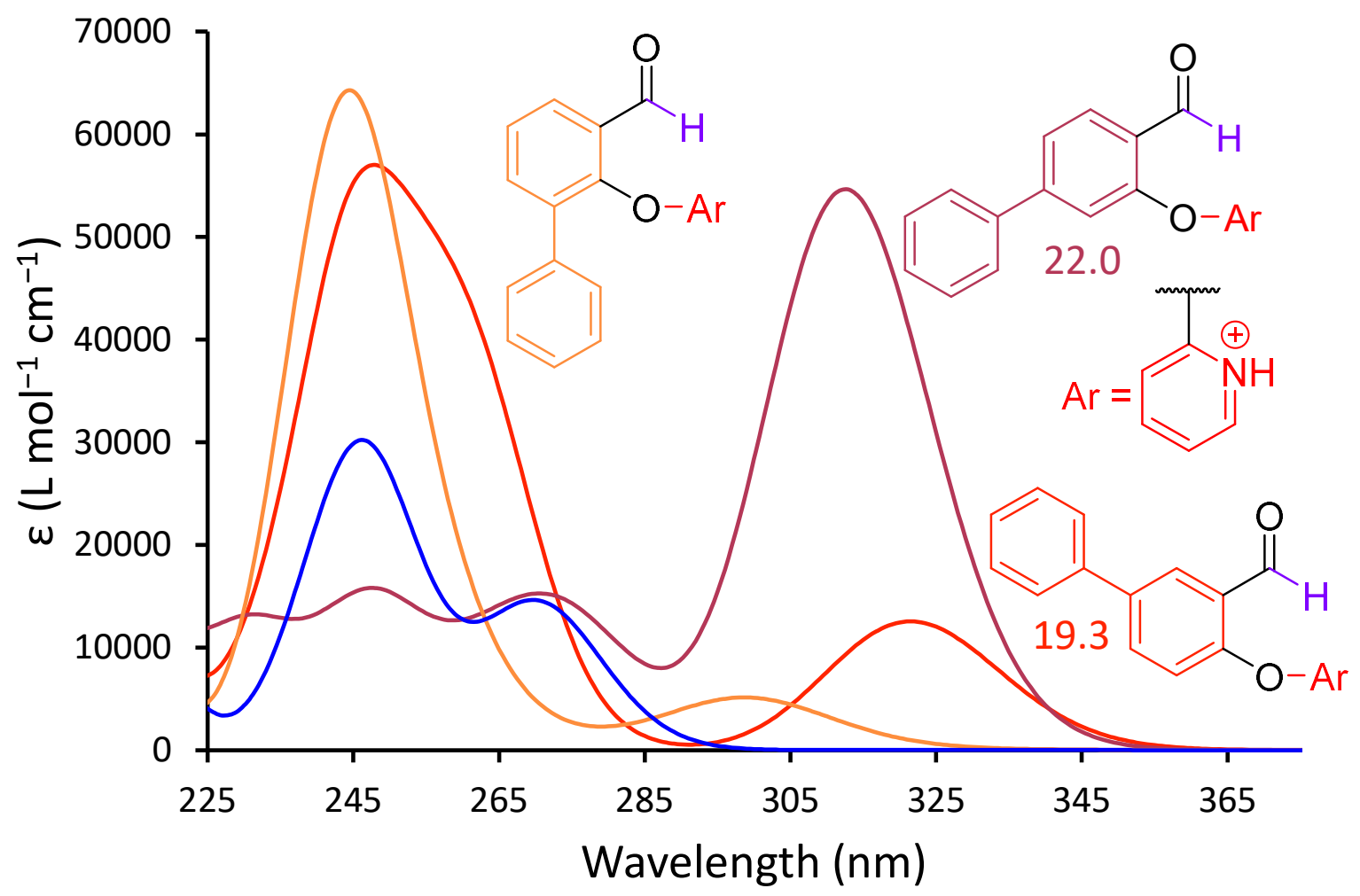

Figure S11. The calculated absorption spectra of the modified (biphenyl) salicylaldehyde ethers. The blue spectrum corresponds to the absorption of $1 \mathbf{a}+$. The numbers below the structures indicate the barrier of the ipso addition. The addition was not calculated for the molecule with the phenyl group ortho to the ether oxygen as it does not offer an improved absorption behavior compared to the unmodified molecule. 


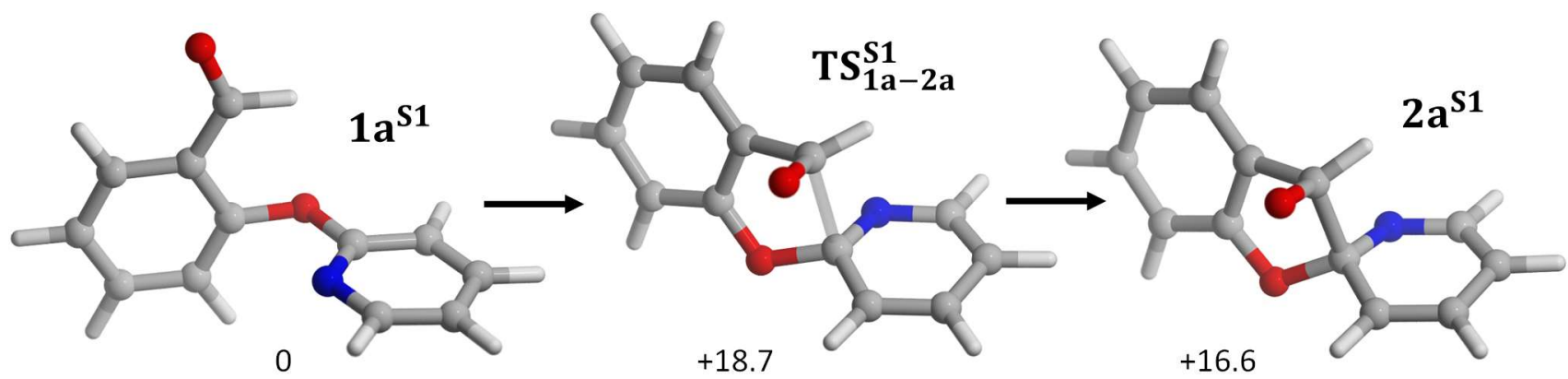

Figure S12. The ipso attack step for $1 \mathrm{a}$ in the $\mathrm{S} 1$ state. The free energies in $\mathrm{kcal} / \mathrm{mol}$ are given relative to the first singlet excited state and are calculated without the def2-TZVPP electronic energy correction.

Coordinates of the TS $\left(v=-279.62 \mathrm{~cm}^{-1}\right)$ :

24

$\begin{array}{rrr}1.19710 & -0.65190 & -0.36500 \\ 1.21010 & 0.63050 & 0.24350 \\ 2.41940 & 1.15470 & 0.70140 \\ 3.56020 & 0.39790 & 0.61300 \\ 3.53030 & -0.87370 & 0.00160 \\ 2.36680 & -1.40280 & -0.49130 \\ -1.10880 & -0.49350 & -0.26070 \\ -0.05440 & 1.38260 & 0.11450 \\ 2.43460 & 2.14490 & 1.14160 \\ 4.49610 & 0.77380 & 1.00810 \\ 4.44940 & -1.44240 & -0.07480 \\ 2.33100 & -2.37610 & -0.96410 \\ 0.06580 & 2.06920 & -0.95110 \\ -1.37820 & -0.86910 & 1.00210 \\ -2.10350 & -0.12520 & -1.19190 \\ -3.33980 & 0.17500 & -0.68590 \\ -3.58660 & 0.00030 & 0.68120 \\ -2.58270 & -0.55810 & 1.45950 \\ -1.85280 & -0.00790 & -2.23810 \\ -4.12620 & 0.52720 & -1.34390 \\ -4.55470 & 0.22010 & 1.11180 \\ -2.77500 & -0.80130 & 2.50210 \\ 0.04420 & -1.12710 & -0.80270 \\ -0.68430 & 1.69460 & 0.95390\end{array}$




\section{Coordinates and energies of the computed structures}

The coordinate file provided alongside the SI is in xyz format and can be directly read by the free Avogadro or Mercury software. The energy values in the second lines (in atomic units, obtained via M06/TZVP opt/freq at $298 \mathrm{~K}$ calculations using the smaller basis set) are in atomic units while the coordinates are in Angstroms. The M06/def2-TZVPP corrected free energies are collected in the tables below.

Table S1. Free energies (a.u.) of the pyridyl ether (1a) used to calculate the $\Delta \mathrm{G}(298 \mathrm{~K})$ values in the main text.

\begin{tabular}{|l|l|l|l|l|l|}
\hline 1a_singlet & -667.547032 & I & -1194.664611 & i_singlet & -1194.363776 \\
\hline 1a_triplet & -667.441705 & TS_I-II & -1194.662053 & i_triplet & -1194.260688 \\
\hline TS_1a-2a & -667.407792 & II & -1194.676449 & ii & -1194.259455 \\
\hline 2a & -667.420159 & TS_II-III* & -1194.677688 & iii & -1194.252614 \\
\hline TS_2a-3a & -667.404751 & III* & -1194.677085 & TS_iii-iv & -1194.227981 \\
\hline 3a & -667.445496 & III & -667.855965 & iv & -1194.236300 \\
\hline TS_3a-4a & -667.425464 & TS_III-IV & -667.855532 & v & -1194.284438 \\
\hline 4a_triplet & -667.484746 & IV & -667.865184 & vi & -1194.284632 \\
\hline 4a_singlet & -667.564632 & TS_IV-3a-prot & -667.857288 & TS_vi-vii & -1194.264749 \\
\hline 1a-prot_singlet & -667.970884 & & & vii_triplet & -1194.320712 \\
\hline 1a-prot_triplet & -667.867677 & TFA & -526.820476 & vii_triplet & -1194.378560 \\
\hline TS_1a-prot-2a-prot & -667.841960 & TFA-H & -526.364919 & TS_ii-iii* & -1194.253712 \\
\hline 2a-prot & -667.848881 & & & iii* & -1194.259358 \\
\hline TS_2a-prot-3a-prot & -667.832697 & & & TS_iii*-v & -1194.257625 \\
\hline 3a-prot & -667.876439 & & & v_singlet & -1194.350606 \\
\hline TS_3a-prot-4a-prot & -667.869334 & & TS_v_singlet-vii* & -1194.347260 \\
\hline 4a-prot_triplet & -667.927504 & & vii* & -1194.356181 \\
\hline 4a-prot_singlet & -667.986109 & & & \\
\hline
\end{tabular}

Table S2. Free energies (a.u.) of the fluorophenyl ether (1b) used to calculate the $\Delta \mathrm{G}(298 \mathrm{~K})$ values in the main text.

\begin{tabular}{|l|l|}
\hline 1b_triplet & -750.634108 \\
\hline TS_1b-2b & -750.604999 \\
\hline 2b & -750.622952 \\
\hline TS_2b-3b & -750.612487 \\
\hline 3b & -750.651491 \\
\hline TS_3b-4b & -750.634534 \\
\hline 4b_triplet & -750.691437 \\
\hline
\end{tabular}


Table S3. Free energies (a.u.) of the pyrazine ether (1c) used to calculate the $\Delta \mathrm{G}(298 \mathrm{~K})$ values in the main text.

\begin{tabular}{|l|l|l|l|l|l|}
\hline 1c_singlet & -762.154636 & 1c_1NH_singlet & -762.567649 & 1c_4NH_singlet & -762.578457 \\
\hline 1c_triplet & -762.049352 & 1c_1NH_triplet & -762.474925 & 1c_4NH_triplet & -762.488318 \\
\hline TS_1c-2c* & -762.025950 & TS_1c_1NH-2c_1NH & -762.449727 & TS_1c_4NH-2c_4NH & -762.450595 \\
\hline 2c* & -762.032974 & 2c_1NH & -762.460666 & 2c_4NH & -762.452615 \\
\hline TS_2c*-3c* & -762.009916 & TS_2c_1NH-3c_1NH & -762.441431 & TS_2c_4NH-3c_4NH & -762.431367 \\
\hline 3c* & -762.052367 & 3c_1NH & -762.474767 & 3c_4NH & -762.471947 \\
\hline TS_3c*44c & -762.036709 & TS_3c_1NH-4c_1NH & -762.457572 & TS_3c_4NH-4c_4NH & -762.462683 \\
\hline 4c_triplet & -762.068631 & 4c_1NH_triplet & -762.520640 & 4c_4NH_triplet & -762.525157 \\
\hline 4c_singlet & -762.163670 & 4c_1NH_singet & -762.583673 & 4c_4NH_singlet & -762.588199 \\
\hline TS_1c-2c & -762.018942 & & & & \\
\hline 2c & -762.033695 & & & & \\
\hline TS_2c-3c & -762.014567 & & & & \\
\hline 3c & -762.049072 & & & & \\
\hline TS_3c-4c & -762.036701 & & & & \\
\hline
\end{tabular}

Table S4. Imaginary (shown as negative, in units $\mathrm{of}^{-1}$ ) frequencies of the TSs. All the TSs have a single imaginary frequency, while the rest of the minima (intermediates, reactants) have no imaginary frequencies.

\begin{tabular}{|l|l|l|l|}
\hline TS_1a-2a & -400.34 & TS_1b-2b & -437.25 \\
\hline TS_2a-3a & -448.16 & TS_2b-3b & -343.1 \\
\hline TS_3a-4a & -1669.68 & TS_3b-4b & -1619.46 \\
\hline TS_1a-prot-2a-prot & -129.19 & TS_1c-2c* & -300.57 \\
\hline TS_2a-prot-3a-prot & -1916.88 & TS_2c*-3c* & -521.59 \\
\hline TS_3a-prot-4a-prot & -1259.94 & TS_3c*-4c & -1573.54 \\
\hline TS_I-II & -911.24 & TS_1c-2c & -339.9 \\
\hline TS_II-III* & -1225.34 & TS_2c-3c & -444.06 \\
\hline TS_III-IV & -289.15 & TS_3c-4c & -1573.58 \\
\hline TS_IV-3a-prot & -826.44 & TS_1c_1NH-2c_1NH & -360.75 \\
\hline TS_iii-iv & -147.43 & TS_2c_1NH-3c_1NH & -1245.53 \\
\hline TS_vi-vii & -1000.01 & TS_3c_1NH-4c_1NH & -1336.72 \\
\hline TS_ii-iii* & -1125.29 & TS_1c_4NH-2c_4NH & -344.25 \\
\hline TS_iii*-v & -43.79 & TS_2c_4NH-3c_4NH & -1082.43 \\
\hline TS_v_singlet-vii* & -329.87 & TS_3c_4NH-4c_4NH & -1335.89 \\
\hline
\end{tabular}




\section{Cartesian coordinates:}

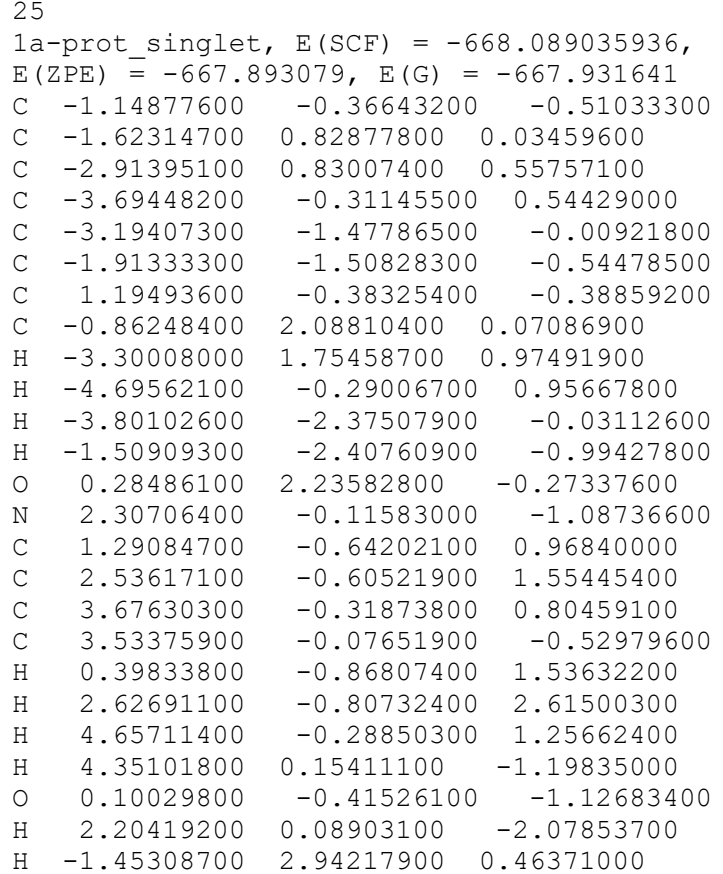

25

1a-prot_triplet, $\mathrm{E}(\mathrm{SCF})=-667.978967489$, $\mathrm{E}(\mathrm{ZPE})=-667.787670, \mathrm{E}(\mathrm{G})=-667.828385$ C $-1.09232500 \quad-0.16830200 \quad-0.55373300$ C $-1.80455100 \quad 0.80439700 \quad 0.17874800$ C $-3.07905200 \quad 0.41791500 \quad 0.66016100$ C $-3.57658500 \quad-0.83721000 \quad 0.40626300$ C $-2.84346400-1.76796500 \quad-0.33099000$ C $-1.58741500-1.42054100-0.81350400$ C $\quad 1.24937700-0.11401700 \quad-0.38834300$ C $-1.28955600 \quad 2.08332200 \quad 0.43388000$ $1.13735200 \quad 1.22809300$ $\mathrm{H}-4.55713100 \quad-1.10406100 \quad 0.78320500$ $\mathrm{H} \quad-3.24590700 \quad-2.75286700 \quad-0.53115800$ $\mathrm{H}-0.99082500-2.11855200 \quad-1.39064400$ $\begin{array}{llll}0 & -0.15230900 & 2.54164000 & 0.00456000\end{array}$ $\begin{array}{llll}\mathrm{N} & 2.38592900 & 0.24055600 & -1.00884700\end{array}$ C $\quad 1.31435200 \quad-0.72671100 \quad 0.85135000$ C $\quad 2.55688000 \quad-0.95379600 \quad 1.40249200$ $\begin{array}{llll}\text { C } & 3.72129700 & -0.57811900 & 0.73497900\end{array}$ $\begin{array}{llll}\text { C } & 3.60805900 & 0.02559700 & -0.48310000\end{array}$ $\mathrm{H} \quad 0.40273000 \quad-1.01158700 \quad 1.35926700$ H $\quad 2.62356500 \quad-1.43002300 \quad 2.37314400$ $\mathrm{H} \quad 4.69885000 \quad-0.75199300 \quad 1.16127500$ H $\quad 4.44621900 \quad 0.35833400 \quad-1.07926300$ o $0.16034100 \quad 0.19549600 \quad-1.07225200$ $\mathrm{H} \quad 2.30721200 \quad 0.69251700 \quad-1.91749500$ $\mathrm{H} \quad-1.85449000 \quad 2.81249200 \quad 1.02781800$

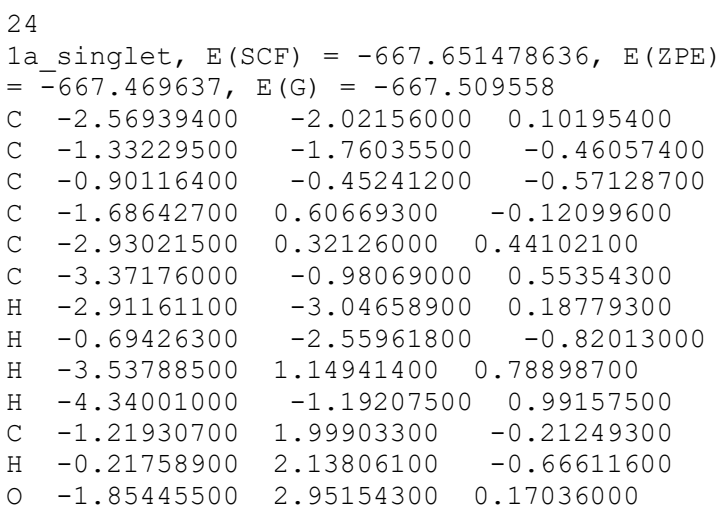

$\begin{array}{llcr}\mathrm{O} & 0.29636400 & -0.20269100 & -1.20961400 \\ \mathrm{C} & 1.43648900 & -0.17086300 & -0.46750400 \\ \mathrm{C} & 2.62753900 & 0.00868700 & -1.16596700 \\ \mathrm{~N} & 1.35119600 & -0.29466900 & 0.83576300 \\ \mathrm{C} & 3.78815800 & 0.05605200 & -0.42772800 \\ \mathrm{H} & 2.61865500 & 0.10488600 & -2.24431400 \\ \mathrm{C} & 2.48846700 & -0.24886000 & 1.53595100 \\ \mathrm{C} & 3.72633700 & -0.07637900 & 0.95613400 \\ \mathrm{H} & 4.74129700 & 0.19423200 & -0.92519700 \\ \mathrm{H} & 2.38942800 & -0.35537200 & 2.61240600 \\ \mathrm{H} & 4.61976700 & -0.04470200 & 1.56636900\end{array}$

24 1 a triplet, $\mathrm{E}(\mathrm{SCF})=-667.541056091, \mathrm{E}(\mathrm{ZPE})$ $=-667.363128, E(G)=-667.404038$

$\begin{array}{cccc}\mathrm{C} & -2.56883700 & -2.03767500 & 0.05660300 \\ \mathrm{C} & -1.33135300 & -1.74330300 & -0.50982300 \\ \mathrm{C} & -0.90183900 & -0.44283500 & -0.58659000 \\ \mathrm{C} & -1.69034200 & 0.62773400 & -0.10332900 \\ \mathrm{C} & -2.94459700 & 0.30519500 & 0.46929200 \\ \mathrm{C} & -3.36417000 & -1.00382300 & 0.54352400 \\ \mathrm{H} & -2.90568800 & -3.06547200 & 0.11683500 \\ \mathrm{H} & -0.68846800 & -2.52726600 & -0.89544300 \\ \mathrm{H} & -3.57376300 & 1.10318800 & 0.84852600 \\ \mathrm{H} & -4.32906100 & -1.22820600 & 0.98463500 \\ \mathrm{C} & -1.21761300 & 1.94490700 & -0.19863200 \\ \mathrm{H} & -0.25858200 & 2.18855300 & -0.67265900 \\ \mathrm{O} & -1.85325600 & 2.99069700 & 0.23237000 \\ \mathrm{O} & 0.29550600 & -0.14931900 & -1.21030500 \\ \mathrm{C} & 1.43532900 & -0.14697800 & -0.46631400 \\ \mathrm{C} & 2.62432100 & 0.07627600 & -1.15677800 \\ \mathrm{~N} & 1.35674700 & -0.34366600 & 0.82872000 \\ \mathrm{C} & 3.78798600 & 0.08938700 & -0.42179600 \\ \mathrm{H} & 2.61131800 & 0.23009900 & -2.22852200 \\ \mathrm{C} & 2.49613500 & -0.32910800 & 1.52569700 \\ \mathrm{C} & 3.73185200 & -0.11861300 & 0.95273900 \\ \mathrm{H} & 4.73862300 & 0.25769700 & -0.91492200 \\ \mathrm{H} & 2.40205800 & -0.49473800 & 2.59524700 \\ \mathrm{H} & 4.62710800 & -0.11619600 & 1.56118300\end{array}$

25

1b_triplet, $\mathrm{E}(\mathrm{SCF})=-750.736085859, \mathrm{E}(\mathrm{ZPE})$ $=-750.554401, \mathrm{E}(\mathrm{G})=-750.594760$

$\begin{array}{llll}\text { C } & -1.26538700 & -0.42719800 & 0.51780600\end{array}$

C $-1.49307500 \quad 0.88917700 \quad 0.04373700$

C $\quad-2.82195500 \quad 1.16862300 \quad-0.37811200$

C $-3.81211600 \quad 0.21906800 \quad-0.34453500$

C $-3.54440500 \quad-1.06860700 \quad 0.12393100$

C $-2.26047400 \quad-1.37435600 \quad 0.55871400$

C $1.12008500 \quad-0.46143900 \quad 0.53792200$

C $\quad-0.53867700 \quad 1.91762900 \quad-0.00851700$

$\mathrm{H} \quad-3.03667900 \quad 2.17023800 \quad-0.73826600$

$\mathrm{H} \quad-4.80924400 \quad 0.47530400 \quad-0.68527200$

$\mathrm{H} \quad-4.32262500 \quad-1.82128700 \quad 0.15475200$

$\mathrm{H}-2.01881400 \quad-2.35642500 \quad 0.95053300$

o $\quad 0.73279600 \quad 1.85438000 \quad 0.24895900$

C $\quad 2.19299400 \quad-0.18214400 \quad 1.39729000$

C $\quad 3.44735600 \quad 0.05269000 \quad 0.88911400$

C $\quad 3.67141100-0.01960200 \quad-0.48386000$

C $2.63787900-0.34500300 \quad-1.35005700$

$\mathrm{H} \quad 1.98452300 \quad-0.13039500 \quad 2.45924100$

$\mathrm{H} \quad 4.26384800 \quad 0.29135500 \quad 1.55924100$

$\mathrm{H} \quad 4.66059600 \quad 0.16859300 \quad-0.88337300$

$\mathrm{H} \quad 2.79431000 \quad-0.43501400 \quad-2.41824700$

$0-0.05446000-0.78822200 \quad 1.09929300$

$\mathrm{H} \quad-0.88090400 \quad 2.92538400 \quad-0.27626900$

C $\quad 1.38732800 \quad-0.58557600 \quad-0.83964000$

F $0.40139100-0.94628600-1.65456700$

30

$1 \mathrm{C} 1 \mathrm{NH}$ singlet, $\mathrm{E}(\mathrm{SCF})=-762.715731573$, $\mathrm{E}(\overline{\mathrm{Z}} \mathrm{PE})=-762.477907, \mathrm{E}(\mathrm{G})=-762.521820$

$\begin{array}{llll}\text { C } & -1.48553400 & 0.00897800 & -0.68233400\end{array}$

C $-1.87568600 \quad 0.88334300 \quad 0.33484300$ 


$\begin{array}{lrcc}\mathrm{C} & -3.21729400 & 0.87012000 & 0.71565300 \\ \mathrm{C} & -4.13214300 & 0.03614600 & 0.10275400 \\ \mathrm{C} & -3.71561900 & -0.79688100 & -0.92291800 \\ \mathrm{C} & -2.38821400 & -0.80692300 & -1.32411300 \\ \mathrm{C} & 0.88135900 & -0.27995600 & -0.45459600 \\ \mathrm{C} & -0.98393000 & 1.82255600 & 1.02628500 \\ \mathrm{H} & -3.53144900 & 1.54129800 & 1.50883800 \\ \mathrm{H} & -5.16848900 & 0.04056100 & 0.41620500 \\ \mathrm{H} & -4.42560600 & -1.44694700 & -1.42006500 \\ \mathrm{H} & -2.04573000 & -1.45148000 & -2.12495600 \\ \mathrm{O} & 0.20870100 & 1.92479000 & 0.85896000 \\ \mathrm{~N} & 1.99107900 & 0.31757400 & -0.89740900 \\ \mathrm{C} & 0.99960100 & -1.18178500 & 0.62000500 \\ \mathrm{C} & 3.25667300 & -0.68237700 & 0.74557100 \\ \mathrm{C} & 3.20831400 & 0.16678800 & -0.33762300 \\ \mathrm{H} & 4.19793700 & -0.85060800 & 1.25647700 \\ \mathrm{O} & -0.18431900 & -0.00185200 & -1.18472500 \\ \mathrm{H} & 1.88810700 & 0.96112300 & -1.68261100 \\ \mathrm{H} & -1.51226400 & 2.47440600 & 1.75169300 \\ \mathrm{~N} & 2.18522700 & -1.33401400 & 1.18282200 \\ \mathrm{C} & 4.34547400 & 0.91154800 & -0.91131300 \\ \mathrm{H} & 5.26302200 & 0.66940200 & -0.37773000 \\ \mathrm{H} & 4.47780700 & 0.66565000 & -1.96790200 \\ \mathrm{H} & 4.17310800 & 1.98858800 & -0.83822700 \\ \mathrm{C} & -0.13775300 & -1.99159100 & 1.13253500 \\ \mathrm{H} & -0.66237100 & -2.50907800 & 0.32658500 \\ \mathrm{H} & 0.25634100 & -2.73207200 & 1.82695600 \\ \mathrm{H} & -0.87110300 & -1.37905900 & 1.66446800 \\ & & -1.650\end{array}$

30

$1 \mathrm{C} 1 \mathrm{NH}$ triplet, $\mathrm{E}(\mathrm{SCF})=-762.618882579$, $\mathrm{E}(\overline{\mathrm{Z}} \mathrm{PE})=-762.385048, \mathrm{E}(\mathrm{G})=-762.428968$

C $-1.49700900-0.32527500 \quad-0.63201600$

C $-1.83993500 \quad 0.89991300 \quad-0.05524200$

C $-3.17282500 \quad 1.08345600 \quad 0.30982100$

C $-4.11728300 \quad 0.09423200 \quad 0.11252000$

C $-3.74410600 \quad-1.09714700 \quad-0.48808900$

$-1.30763400-0.87300200$

C $\quad 0.85223600-0.40703700 \quad-0.35036600$

C $\quad-0.92421300 \quad 2.03140600 \quad 0.13022600$

$\mathrm{H} \quad-3.45622800 \quad 2.03410900 \quad 0.74980300$

$\mathrm{H} \quad-5.14516600 \quad 0.25618200 \quad 0.41164400$

$\mathrm{H}-4.47911400 \quad-1.87313900 \quad-0.66400700$

$\mathrm{H} \quad-2.11785000 \quad-2.22981500 \quad-1.34952700$

$0.26439500 \quad 2.02397800 \quad-0.09752900$

$1.98912200-0.24800200-1.04140400$

$0.92522100 \quad-0.57842000 \quad 1.05591100$

$\begin{array}{lll}3.13783300 & 0.01392800 & 1.04260200\end{array}$

$3.21376400-0.14398000-0.35821100$

$\begin{array}{llll}4.04539300 & 0.32074900 & 1.55873600\end{array}$

$-0.20557400-0.58682000-1.10000200$

$\begin{array}{llll}1.93861600 & 0.00709700 & -2.02290700\end{array}$

$\begin{array}{llll}-1.42206700 & 2.94970700 & 0.50008700\end{array}$

$\begin{array}{lll}2.04205100 & -0.09676400 & 1.73367900\end{array}$

$\begin{array}{llll}4.41975800 & 0.03052300 & -1.17145200\end{array}$

$5.30431500 \quad 0.11010000-0.54143100$

$4.54907800-0.81249300-1.85985100$

$4.34935500 \quad 0.93301100 \quad-1.79256600$

$\begin{array}{lll}-0.05192500 & -1.33244600 & 1.85387800\end{array}$

$-0.68592800 \quad-1.99426700 \quad 1.26595100$

$0.49140400-1.91297900 \quad 2.60531100$

$\mathrm{H} \quad-0.69798700 \quad-0.64128500 \quad 2.41360500$

\section{0}

1C_4NH_singlet, $\mathrm{E}(\mathrm{SCF})=-762.723645988$, $\mathrm{E}(\overline{\mathrm{Z}} \mathrm{PE})=-762.485696, \mathrm{E}(\mathrm{G})=-762.532562$

3.00667200

$0.71378100-1.83472000$

$\begin{array}{lllr}\text { C } & 1.75653600 & 0.11661400 & -1.81901900 \\ \text { C } & 1.28498300 & -0.40375500 & -0.63442200\end{array}$

$\begin{array}{llll}\text { C } & 2.01998300 & -0.34479400 & 0.54501200\end{array}$

$\begin{array}{llll}\text { C } & 3.27353500 & 0.26281600 & 0.50357700\end{array}$

C $\quad 3.76533400 \quad 0.78739200 \quad-0.67452600$

$\mathrm{H} \quad 3.39041000 \quad 1.12256800 \quad-2.76197300$

$\mathrm{H} \quad 1.15103200 \quad 0.04741000 \quad-2.71505900$

$\begin{array}{llll}\mathrm{H} & 3.85179000 & 0.30768500 & 1.41961300\end{array}$

$\mathrm{H} \quad 4.74315000 \quad 1.25304400 \quad-0.69497600$

C $1.50972600 \quad-0.90111800 \quad 1.81005700$

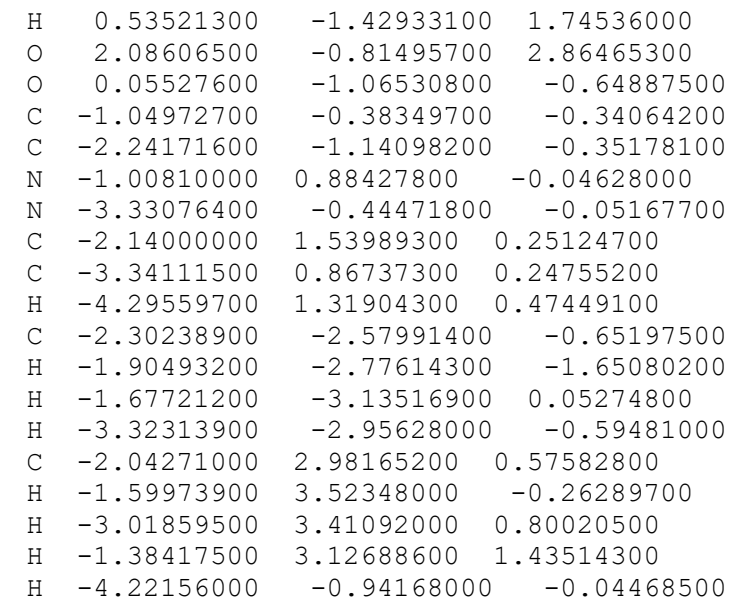

30

$1 \mathrm{C} 4 \mathrm{NH}$ triplet, $\mathrm{E}(\mathrm{SCF})=-762.631135444$, $\mathrm{E}(\overline{\mathrm{Z}} \mathrm{PE}){ }^{-}=-762.397509, \mathrm{E}(\mathrm{G})=-762.441992$

$\begin{array}{lll}031900 & 0.00333800 & 0.82864600\end{array}$

$\begin{array}{lllll}\text { C } & -3.09384600 & 0.51530300 & 0.99943700\end{array}$

C $\quad-4.00166900 \quad 0.53807300 \quad-0.04357100$

C $\quad-3.64114000 \quad 0.02107700 \quad-1.27723000$

C $-2.37517600 \quad-0.51129300 \quad-1.47467100$

C $0.85870900 \quad-0.42902400 \quad-0.48406000$

C $-0.92810200 \quad-0.02606600 \quad 2.00234700$

$\mathrm{H} \quad-3.37013200 \quad 0.89875500 \quad 1.97683500$

$\mathrm{H} \quad-4.99202700 \quad 0.94981000 \quad 0.10571700$

$\mathrm{H} \quad-4.34923700 \quad 0.02542100 \quad-2.09732800$

$\mathrm{H} \quad-2.07925600 \quad-0.92916800 \quad-2.42923200$

$0 \quad 0.22092500 \quad-0.40376000 \quad 2.03175500$

$\mathrm{N} \quad 0.80946800 \quad 0.89717500 \quad-0.55683300$

C $2.01942500 \quad-1.21988400 \quad-0.28357800$

C $\quad 3.16657500 \quad 0.85217000 \quad-0.05804700$

C $\quad 1.97294900 \quad 1.53468000 \quad-0.32476100$

$\begin{array}{llll}\mathrm{H} & 4.09161000 & 1.37903200 & 0.13605600\end{array}$

O $-0.25069000-1.11816000 \quad-0.64656200$

$\begin{array}{llll}\mathrm{H} & -1.42262400 & 0.33548900 & 2.92697900\end{array}$

C $\quad 1.95876400 \quad 3.00606600 \quad-0.38806000$

$\mathrm{H} \quad 1.55568000 \quad 3.32753600 \quad-1.35355200$

$\mathrm{H}-1.27257300$

$\mathrm{H} \quad 2.94545500 \quad 3.44187800 \quad-0.23972500$

C $2.05029800 \quad-2.66631700 \quad-0.07847400$

H $2.90206800 \quad-3.10789000 \quad-0.60675700$

$\mathrm{H} \quad 2.19471900-2.88665900 \quad 0.98912000$

H $\quad 1.13204100-3.14836300 \quad-0.40496300$

$\mathrm{N} \quad 3.20556200 \quad-0.50094900 \quad-0.08021500$

$\begin{array}{llll}\mathrm{H} & 4.06634100 & -1.00088200 & 0.09327000\end{array}$

\section{9}

1C_singlet, $\mathrm{E}(\mathrm{SCF})=-762.290630039, \mathrm{E}(\mathrm{ZPE})$

$=-762.066233, \mathrm{E}(\mathrm{G})=-762.110801$

C $\quad 2.95350500 \quad 0.90250700-1.76050800$

C $1.71294200 \quad 0.28938300-1.79646000$

C $1.24418300 \quad-0.35002200 \quad-0.66636700$

C $\quad 1.99086500 \quad-0.38694600 \quad 0.50908800$

$\begin{array}{llll}\text { C } & 3.23731200 & 0.23751200 & 0.52281700\end{array}$

C $\quad 3.71804500 \quad 0.87788900 \quad-0.60067200$

$\mathrm{H} \quad 3.32723400 \quad 1.40374000 \quad-2.64600500$

$\mathrm{H} \quad 1.10296400 \quad 0.29819400 \quad-2.69241700$

$\mathrm{H} \quad 3.81695500 \quad 0.20595400 \quad 1.43877900$

$\mathrm{H} \quad 4.68866800 \quad 1.35888000 \quad-0.58003300$

$\mathrm{C} \quad 1.48678100 \quad-1.05820900 \quad 1.71822400$

$\mathrm{H} \quad 0.49837900-1.55144900 \quad 1.61565700$

$\begin{array}{llll}0 & 2.08089100 & -1.09452700 & 2.76789200\end{array}$

$0 \quad 0.03700800 \quad-1.01988100 \quad-0.73694300$

C $-1.09236000-0.36373400-0.36964800$

C $-2.28906900-1.10431900-0.41462000$

$\begin{array}{llll}\mathrm{N} & -1.02895500 & 0.88314200 & 0.00593100\end{array}$

$\mathrm{N}-3.40928700 \quad-0.49867100 \quad-0.07117300$

C $-2.17489700 \quad 1.48811600 \quad 0.35541500$

C $-3.36055400 \quad 0.78056800 \quad 0.30599900$ 
$1.25436800 \quad 0.58209500$ $-2.66325700-1.83922100$ $-3.14024700-0.16819700$ $-2.91228800 \quad-0.78073200$ $2.90870700 \quad 0.78549800$

$3.52539100-0.00469600$

$3.30420200 \quad 1.03247900$

$3.00874500 \quad 1.66235700$
$-2.53282900 \quad-0.82392500$

29

1C_triplet, $\mathrm{E}(\mathrm{SCF})=-762.182098363, \mathrm{E}(\mathrm{ZPE})$

$=-761.961070, \mathrm{E}(\mathrm{G})=-762.005372$

$\begin{array}{llll}\text { C } & -1.36296000 & -0.27460800 & -0.53246900\end{array}$

C $\quad-2.09722600 \quad-0.50565200 \quad 0.65246100$

$\begin{array}{llll}\text { C } & -3.34994700 & 0.14140800 & 0.77284500\end{array}$

C $-3.82096500 \quad 0.94830200 \quad-0.23572300$

C $-3.07784900 \quad 1.14840600-1.39926500$

C $-1.83969800 \quad 0.52952400-1.53747200$

C $\quad 0.99203000 \quad-0.27089000 \quad-0.35552500$

C $-1.59725300 \quad-1.34286000 \quad 1.65958400$

$\mathrm{H}-3.93421700-0.01646200-1.67305700$

$\mathrm{H} \quad-4.78524800 \quad 1.43132900 \quad-0.12362500$

$\mathrm{H} \quad-3.45622800 \quad 1.78346700 \quad-2.19098600$

$\mathrm{H} \quad-1.23363700 \quad 0.67476300 \quad-2.42527200$

$\begin{array}{llll}0 & -0.48629000 & -2.01551000 & 1.59923900\end{array}$

$\begin{array}{llll}\mathrm{N} & 0.93891500 & 0.94974900 & 0.09804200\end{array}$

C $\quad 2.19160000 \quad-0.98612100 \quad-0.53370800$

$\begin{array}{llll}\text { C } & 3.28851800 & 0.87174400 & 0.21406000\end{array}$

$\begin{array}{llll}\text { C } & 2.09973300 & 1.55353300 & 0.39417900\end{array}$

H $\quad 4.23734400 \quad 1.34662600 \quad 0.44614500$

$\begin{array}{llll}0 & -0.15120200 & -0.93151100 & -0.67442600\end{array}$

$\mathrm{H} \quad-2.14776800 \quad-1.49546100 \quad 2.59514400$

$\begin{array}{lll}2.03821600 & 2.94568800 & 0.91136300\end{array}$

$\begin{array}{lll}1.44927100 & 2.98598000 & 1.83134600\end{array}$

$3.03429400 \quad 3.33889100 \quad 1.11822300$

$\begin{array}{lll}1.54737400 & 3.60169200 & 0.18781000\end{array}$

$2.19466100 \quad-2.38910700 \quad-1.02186700$

$3.21690700 \quad-2.76559000 \quad-1.05154100$

$\begin{array}{lll}1.59548700 & -3.03172800 & -0.37023600\end{array}$

$\begin{array}{lll}1.75968900 & -2.46486600 & -2.02238500\end{array}$

$\begin{array}{lll}3.32730100 & -0.38241700 & -0.24046500\end{array}$

25

$2 \mathrm{a}$-prot, $\mathrm{E}(\mathrm{SCF})=-667.966888282, \mathrm{E}(\mathrm{ZPE})=$ $-667.773111, E(G)=-667.811707$

C $-1.21084200-0.60400800-0.43752700$

C $-1.33204800 \quad 0.51220700 \quad 0.38424200$

C $-2.56985600 \quad 0.93522200 \quad 0.84294800$

C $-3.67167000 \quad 0.16825800 \quad 0.51674000$

$\begin{array}{llll}\text { C } & -3.53267100 & -0.96320900 & -0.28908100\end{array}$

C $-2.30060300-1.37380500-0.77892900$

C $\quad 0.94156100-0.01635600 \quad-0.05030600$

$\begin{array}{lrrr}\text { C } & 0.02233600 & 1.17036300 & 0.45374800\end{array}$

$\mathrm{H} \quad-2.65893600 \quad 1.83351800 \quad 1.44235600$

H $\quad-4.65297500 \quad 0.44950000 \quad 0.87810800$

$\mathrm{H} \quad-4.41358500 \quad-1.54312300 \quad-0.53953300$

$\mathrm{H}-2.19621200-2.25368800-1.40090800$

$\begin{array}{llll}0 & -0.14766700 & 2.12258400 & -0.48305500\end{array}$

$\mathrm{N} \quad 1.94070600 \quad 0.49111800 \quad-0.94944700$

$\begin{array}{llll}\text { C } & 1.52484700 & -0.79080300 & 1.06460600\end{array}$

$\begin{array}{llll}\text { C } & 2.86777900 & -0.96200100 & 1.20970800\end{array}$

C $\quad 3.74811700 \quad-0.40683600 \quad 0.27784400$

C $\quad 3.22855600 \quad 0.32087700 \quad-0.80037200$

H $\quad 0.81335700 \quad-1.22012400 \quad 1.76280900$

$\mathrm{H} \quad 3.25412000 \quad-1.54210700 \quad 2.03849300$

$\mathrm{H} \quad 4.81913800 \quad-0.52821400 \quad 0.35952800$

H $\quad 3.86976700 \quad 0.77377400 \quad-1.54733600$

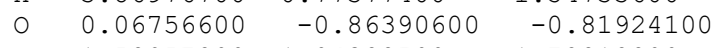

$\mathrm{H} \quad 1.58957800 \quad 1.04388500 \quad-1.72818800$

$\mathrm{H} \quad 0.33856400 \quad 1.53988300 \quad 1.43743500$

24

$2 \mathrm{a}, \mathrm{E}(\mathrm{SCF})=-667.525878390, \mathrm{E}(\mathrm{ZPE})=-$ $667.345756, \mathrm{E}(\mathrm{G})=-667.384475$

$\begin{array}{llll}\text { C } & 1.20580000 & -0.61773700 & -0.45128200\end{array}$

C $\quad 1.25606000 \quad 0.53910900 \quad 0.32184700$

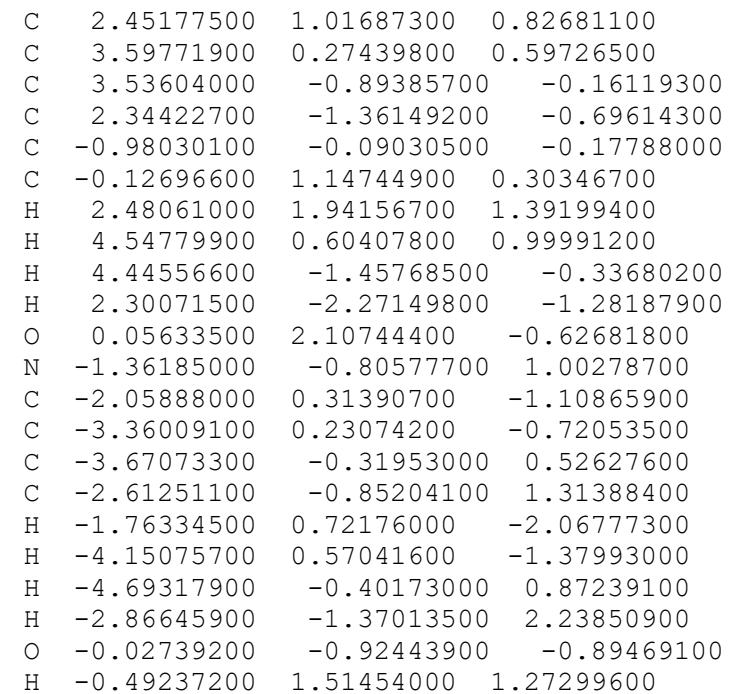

25

$2 \mathrm{~b}, \mathrm{E}(\mathrm{SCF})=-750.729984792, \mathrm{E}(\mathrm{ZPE})=-$

$750.546043, E(G)=-750.585621$

$\begin{array}{llcc}\mathrm{C} & -1.25417100 & -0.27374900 & 0.72965300 \\ \mathrm{C} & -1.39472000 & 0.41112500 & -0.47367000 \\ \mathrm{C} & -2.63278500 & 0.57959600 & -1.06809100 \\ \mathrm{C} & -3.73127900 & -0.00431200 & -0.46150800 \\ \mathrm{C} & -3.58076800 & -0.70234900 & 0.73635500 \\ \mathrm{C} & -2.34643100 & -0.85129600 & 1.35118800 \\ \mathrm{C} & 0.91302200 & 0.21246900 & 0.14664700 \\ \mathrm{C} & -0.04687300 & 0.99607000 & -0.82261500 \\ \mathrm{H} & -2.72887400 & 1.14951800 & -1.98537100 \\ \mathrm{H} & -4.71274400 & 0.08765700 & -0.91052300 \\ \mathrm{H} & -4.45365800 & -1.14657100 & 1.20187900 \\ \mathrm{H} & -2.23434300 & -1.39817100 & 2.27935100 \\ \mathrm{O} & -0.26789800 & 2.28282900 & -0.46742200 \\ \mathrm{C} & 1.90615300 & 1.09180100 & 0.81276800 \\ \mathrm{C} & 3.23929600 & 0.84315400 & 0.78313800 \\ \mathrm{C} & 3.76824600 & -0.26927500 & 0.10091500 \\ \mathrm{C} & 2.90054100 & -1.15607600 & -0.56245400 \\ \mathrm{H} & 1.49970700 & 1.94859100 & 1.33655700 \\ \mathrm{H} & 3.91586700 & 1.51883300 & 1.29456200 \\ \mathrm{H} & 4.83546100 & -0.45176100 & 0.09084900 \\ \mathrm{H} & 3.27756300 & -2.02168000 & -1.09502000 \\ \mathrm{O} & 0.00905100 & -0.34165300 & 1.18246100 \\ \mathrm{H} & 0.25100900 & 0.87126900 & -1.87334100 \\ \mathrm{C} & 1.57120400 & -0.92571200 & -0.53652700 \\ \mathrm{~F} & 0.72802000 & -1.75508600 & -1.16378200 \\ & & & \\ & -0.950 & \end{array}$

29

$2 \mathrm{C}, \mathrm{E}(\mathrm{SCF})=-762.172519243, \mathrm{E}(\mathrm{ZPE})=-$

$761.949722, E(G)=-761.991867$

$\begin{array}{lrcc}\mathrm{C} & 1.55377900 & 0.12347800 & -0.71335000 \\ \mathrm{C} & 1.52261000 & -0.21161600 & 0.63704800 \\ \mathrm{C} & 2.66214500 & -0.62405500 & 1.30328100 \\ \mathrm{C} & 3.82881900 & -0.76128300 & 0.57041500 \\ \mathrm{C} & 3.84639800 & -0.44433000 & -0.78704700 \\ \mathrm{C} & 2.71344600 & 0.00119500 & -1.45307700 \\ \mathrm{C} & -0.64149300 & 0.23067800 & -0.21820700 \\ \mathrm{C} & 0.14186500 & 0.12824800 & 1.15264600 \\ \mathrm{H} & 2.63234600 & -0.84017900 & 2.36516700 \\ \mathrm{H} & 4.73438000 & -1.10889800 & 1.05261100 \\ \mathrm{H} & 4.77170000 & -0.55136600 & -1.34214700 \\ \mathrm{H} & 2.73007500 & 0.24250900 & -2.50880200 \\ \mathrm{O} & 0.39772400 & 1.29963600 & 1.76602800 \\ \mathrm{~N} & -1.15209500 & -1.07554400 & -0.51186600 \\ \mathrm{C} & -1.67725700 & 1.31014400 & -0.17777900 \\ \mathrm{C} & -3.18913400 & -0.27804600 & 0.41499100 \\ \mathrm{C} & -2.36226500 & -1.31726700 & -0.13345400 \\ \mathrm{H} & -4.14864100 & -0.51491500 & 0.86540000 \\ \mathrm{O} & 0.36730100 & 0.54213800 & -1.19761800 \\ \mathrm{H} & -0.31987500 & -0.63088700 & 1.80115500 \\ \mathrm{C} & -2.94392800 & -2.67479900 & -0.31712900 \\ \mathrm{H} & -2.19455800 & -3.36375100 & -0.70568100 \\ & & & \end{array}$




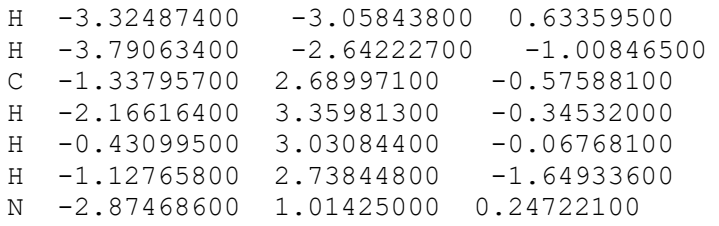

29

$\begin{array}{lrcc}2 C^{*}, E(\mathrm{SCF})=-762.170646971, & E(\mathrm{ZPE})=- \\ 761.948880, \mathrm{E}(\mathrm{G}) & =-761.991156 & \\ \mathrm{C} & 1.61917300 & 0.35244700 & -0.63757100 \\ \mathrm{C} & 1.58762900 & -0.22476900 & 0.61890900 \\ \mathrm{C} & 2.72191100 & -0.77632600 & 1.17431800 \\ \mathrm{C} & 3.89715300 & -0.74678300 & 0.43354300 \\ \mathrm{C} & 3.91823200 & -0.15763900 & -0.82585300 \\ \mathrm{C} & 2.77732100 & 0.40182500 & -1.38644600 \\ \mathrm{C} & -0.59940300 & 0.35809400 & -0.16662800 \\ \mathrm{C} & 0.19357200 & -0.06137900 & 1.15378200 \\ \mathrm{H} & 2.69034300 & -1.22849500 & 2.15990700 \\ \mathrm{H} & 4.80226900 & -1.18441800 & 0.83777200 \\ \mathrm{H} & 4.84426100 & -0.14022200 & -1.38961600 \\ \mathrm{H} & 2.78740800 & 0.85346300 & -2.37113800 \\ \mathrm{O} & -0.36836400 & -0.98841600 & 1.91484100 \\ \mathrm{~N} & -1.13661900 & -0.84082300 & -0.73049000 \\ \mathrm{C} & -1.59532200 & 1.43303600 & 0.13021100 \\ \mathrm{C} & -3.21958300 & -0.15172100 & 0.18806500 \\ \mathrm{C} & -2.37888800 & -1.09445800 & -0.49173600 \\ \mathrm{H} & -4.23539900 & -0.42071900 & 0.46241600 \\ \mathrm{O} & 0.42166200 & 0.85898500 & -1.03857900 \\ \mathrm{H} & 0.20075400 & 0.83583200 & 1.83008400 \\ \mathrm{~N} & -2.83421600 & 1.11882500 & 0.38532300 \\ \mathrm{C} & -2.98136100 & -2.36637100 & -0.97788900 \\ \mathrm{H} & -3.79982900 & -2.16708400 & -1.67496600 \\ \mathrm{H} & -2.23054400 & -2.98038900 & -1.47417900 \\ \mathrm{H} & -3.40493300 & -2.92851100 & -0.14077600 \\ \mathrm{C} & -1.14487400 & 2.83951100 & 0.20664700 \\ \mathrm{H} & -0.90080700 & 3.21944300 & -0.79065300 \\ \mathrm{H} & -1.92801400 & 3.46123900 & 0.64001500 \\ \mathrm{H} & -0.22940500 & 2.93648800 & 0.80109900 \\ & & & \end{array}$

30

$2 \mathrm{C} 1 \mathrm{NH}, \mathrm{E}(\mathrm{SCF})=-762.610089041, \mathrm{E}(\mathrm{ZPE})=-$

$76 \overline{2} .374801, E(G)=-762.417040$

C $\quad 1.64425100 \quad 0.30655700 \quad-0.6479$

1.62167700

2.76390300

3.92844800

3.93512800

2.78645200

$-0.56123500$

0.22613100

2.74666700

4.84081900

4.85590500

2.78273100

$-0.33863000$

$\mathrm{N}-1.23005000$

$-1.55463000$

C -3.25432100

C -2.48987200

$\mathrm{H} \quad-4.27489600$

0.42077200

$-0.64875100$

0.23522300

$-2.79814800$

$-3.07499900$

$-4.01494400$

$-2.39296900$

H $\quad-3.31234700$

C -1.05732700

$-0.87575100$

$-1.78808800$

$\mathrm{H} \quad-0.09498900$

49810

$-0.20548100 \quad 0.63211100$

$-0.72231800 \quad 1.20698700$

$-0.71965400 \quad 0.45145500$

$-0.19643200-0.83676000$

$0.32719700-1.41565800$

$0.38123500-0.16008400$

$-0.03614100 \quad 1.17486500$

$-1.12213100 \quad 2.21464700$

$-1.12759700 \quad 0.86904900$

$-0.20127800-1.40826700$

$0.72986800-2.42106700$

$-0.98092600 \quad 1.90586000$

$-0.79357600 \quad-0.68255900$

$\begin{array}{lll}1.46435300 & 0.12102400\end{array}$

$\begin{array}{ll}-0.06257800 & 0.20632100\end{array}$

$-1.06416300-0.44534300$

$\begin{array}{ll}-0.28389100 & 0.49761900\end{array}$

$0.78107300-1.07536000$

$-1.47973900 \quad-1.16108100$

$0.85394900 \quad 1.85803100$

$\begin{array}{lll}1.18656000 & 0.37915100\end{array}$

$-2.34183000-0.87979900$

$-2.16654500-1.40655300$

$-2.90328900-1.51786400$

$-2.94220300 \quad 0.00414300$

$2.84824700 \quad 0.16957300$

$3.21136200-0.84849900$

$3.49543000 \quad 0.65094900$

$2.91003700 \quad 0.68848900$
$2 \mathrm{C} 4 \mathrm{NH}, \mathrm{E}(\mathrm{SCF})=-762.602889478, \mathrm{E}(\mathrm{ZPE})=-$ $76 \overline{2} .366789, E(G)=-762.408728$

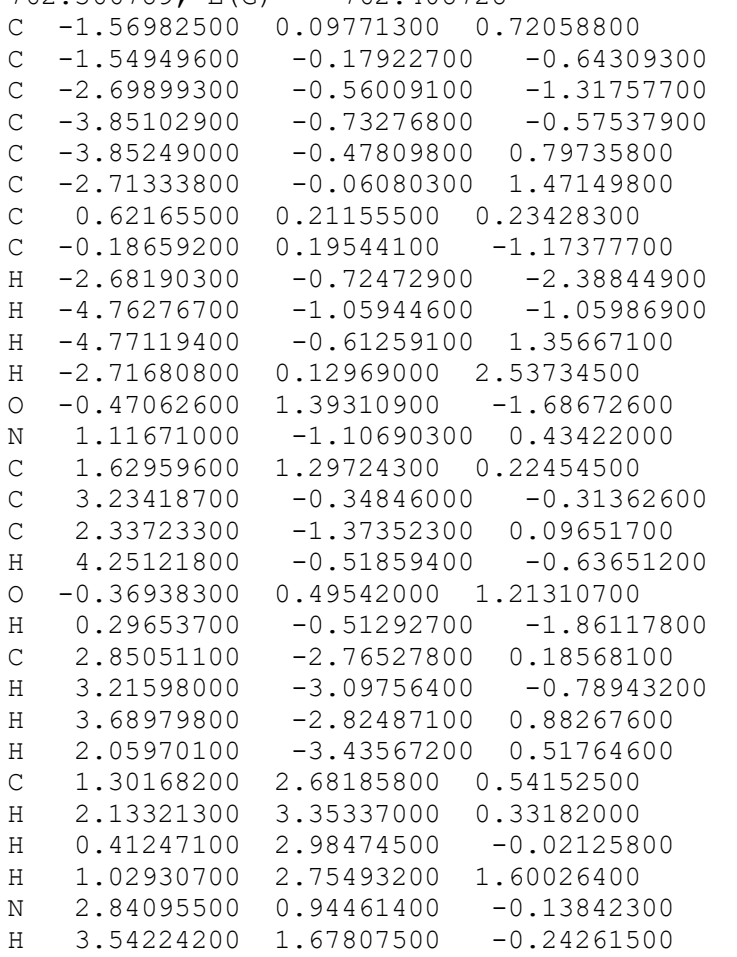

25

3a-prot, $\mathrm{E}(\mathrm{SCF})=-667.990979193, \mathrm{E}(\mathrm{ZPE})=$ $-667.798753, E(G)=-667.840544$

C $\quad 2.09132500 \quad 0.02629700 \quad 0.96039000$

C 1.15642800

C $1.43324300 \quad 0.14613600 \quad-1.40267400$

C $2.63858900 \quad-0.47843800 \quad-1.75556800$

$\begin{array}{llll}\text { C } & 3.56826600 & -0.85140700 & -0.77575600\end{array}$

C $\quad 3.31921500 \quad-0.61152500 \quad 0.54494600$

C $-1.36285000 \quad 0.23737100 \quad 0.18842700$

C $-0.11985900 \quad 1.08702000 \quad 0.35710100$

$\mathrm{H} \quad 0.73045900 \quad 0.43036100 \quad-2.17905800$

$\mathrm{H} \quad 2.85375500 \quad-0.66590800 \quad-2.80100800$

$\mathrm{H} \quad 4.49288000-1.33040600-1.07561400$

$\mathrm{H} \quad 4.02004300 \quad-0.89252100 \quad 1.32255300$

$\begin{array}{llll}0 & -0.24319200 & 2.31712000 & -0.14869900\end{array}$

$\mathrm{N}-2.44535600 \quad 0.86803300 \quad-0.28215000$

C $-1.46009500 \quad-1.09958100 \quad 0.50782900$

C $-2.67156100 \quad-1.74603800 \quad 0.33463700$

C $-3.76913200-1.05668100 \quad-0.16362800$

$\begin{array}{llll}\text { C } & -3.63206700 & 0.27237800 & -0.46997700\end{array}$

$\mathrm{H}-0.59523700 \quad-1.62488800 \quad 0.89396000$

$\mathrm{H}-2.76161200 \quad-2.79598700 \quad 0.58543400$

$\mathrm{H}-4.72252800 \quad-1.54460000-0.31027400$

$\mathrm{H}-4.42868400 \quad 0.88868800 \quad-0.86293100$

$\begin{array}{llll}0 & 1.82494200 & 0.24014000 & 2.15254800\end{array}$

$\mathrm{H} \quad-2.32976600 \quad 1.85770500 \quad-0.51046500$

$\mathrm{H} \quad 0.05516600 \quad 1.20153400 \quad 1.46672300$
24

$3 \mathrm{a}, \mathrm{E}(\mathrm{SCF})=-667.550192237, \mathrm{E}(\mathrm{ZPE})=-$

$667.371010, E(G)=-667.411215$

$\begin{array}{llll}\text { C } & -1.66549900 & 0.85781600 & 0.46759600\end{array}$

C $-1.14104300-0.00534000 \quad-0.58149500$

C $-1.74743400 \quad-1.19866500 \quad-0.86305700$

C $-2.87834000 \quad-1.60484500 \quad-0.14709600$

$\begin{array}{llll}C & -3.41310600 & -0.80151000 & 0.87149000\end{array}$

C $-2.83686900 \quad 0.39583700 \quad 1.17768200$

C $\quad 1.35210400 \quad 0.06961600 \quad-0.47985800$

C $\quad 0.11691500 \quad 0.48224900 \quad-1.26446600$

$\mathrm{H}-1.34854700 \quad-1.83551400 \quad-1.64542100$

$\mathrm{H}-3.34875700 \quad-2.55199500 \quad-0.38529100$

$\mathrm{H} \quad-4.29215100 \quad-1.13780600 \quad 1.40926500$

$\mathrm{H}-3.22823300 \quad 1.04065900 \quad 1.95661600$ 


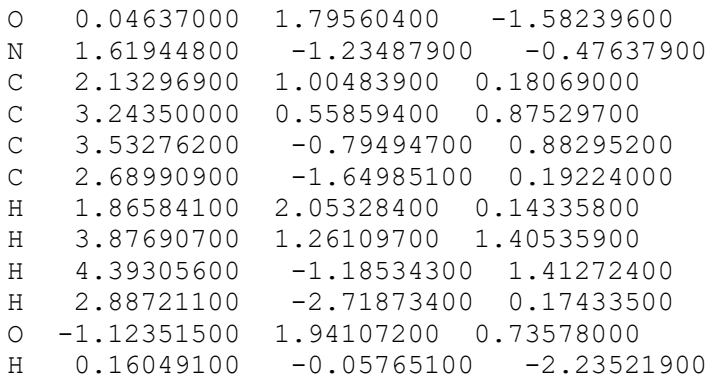

25

$\begin{array}{lccc}3 \mathrm{~b}, \mathrm{E}(\mathrm{SCF})=-750.755567419, \mathrm{E}(\mathrm{ZPE})=- \\ 750.573617, \mathrm{E}(\mathrm{G})=-750.615534 \\ \mathrm{C} & -2.27991600 & 0.69306400 & 0.37559000 \\ \mathrm{C} & -1.16862500 & -0.24638100 & 0.31444000 \\ \mathrm{C} & -1.23464700 & -1.35178800 & -0.49039700 \\ \mathrm{C} & -2.37530000 & -1.59937400 & -1.26019000 \\ \mathrm{C} & -3.46815800 & -0.71885300 & -1.22920900 \\ \mathrm{C} & -3.43631200 & 0.39206700 & -0.43954400 \\ \mathrm{C} & 1.34945500 & -0.03462300 & 0.49834300 \\ \mathrm{C} & 0.02367000 & 0.01448100 & 1.21727200 \\ \mathrm{H} & -0.39837300 & -2.04350900 & -0.52989900 \\ \mathrm{H} & -2.41549500 & -2.48372100 & -1.88574200 \\ \mathrm{H} & -4.34048100 & -0.93269800 & -1.83617600 \\ \mathrm{H} & -4.26374300 & 1.09172600 & -0.39931700 \\ \mathrm{O} & -0.05941400 & -0.74186200 & 2.32817700 \\ \mathrm{C} & 2.27637200 & -1.04090300 & 0.73548400 \\ \mathrm{C} & 3.47815800 & -1.07719700 & 0.04667000 \\ \mathrm{C} & 3.76897300 & -0.10200200 & -0.89508200 \\ \mathrm{C} & 2.85939100 & 0.91218800 & -1.15005000 \\ \mathrm{H} & 2.04405300 & -1.79541600 & 1.47912100 \\ \mathrm{H} & 4.18912000 & -1.87081300 & 0.24424700 \\ \mathrm{H} & 4.70815500 & -0.12624000 & -1.43524300 \\ \mathrm{H} & 3.05733500 & 1.69122500 & -1.87667900 \\ \mathrm{O} & -2.22631000 & 1.70897600 & 1.08444300 \\ \mathrm{H} & -0.15959200 & 1.05906300 & 1.58294300 \\ \mathrm{C} & 1.67234200 & 0.92072900 & -0.45175600 \\ \mathrm{~F} & 0.78582200 & 1.90055800 & -0.69707100\end{array}$

29

$761.963764, E(G)=-762.008712$

C $\quad-1.63938600 \quad 1.25519900 \quad-0.18444600$

C $-1.53761000 \quad 0.03995900 \quad 0.61182700$

$\begin{array}{llll}\text { C } & -2.62016900 & -0.78913400 & 0.73197700\end{array}$

C -3.81188600

C $-3.93086600 \quad 0.62121400 \quad-0.76227300$

C $-2.88249400 \quad 1.48385800 \quad-0.88789100$

C $\quad 1.00786300 \quad-0.27126500 \quad 0.39611900$

C $-0.22342700 \quad-0.24423700 \quad 1.30198000$

$\mathrm{H}-2.54532300 \quad-1.67901800 \quad 1.34714600$

$\mathrm{H} \quad-4.65281800 \quad-1.18929900 \quad 0.16009600$

$\mathrm{H} \quad-4.86140600 \quad 0.80793800 \quad-1.28604600$

$\mathrm{H} \quad-2.94316200 \quad 2.37874100 \quad-1.49756700$

o $-0.24240900 \quad-1.31949900 \quad 2.12205700$

$\begin{array}{llll}\mathrm{N} & 2.02836700 & 0.47524000 & 0.78339000\end{array}$

C $\quad 1.07960300-1.09428100 \quad-0.73506700$

C $3.19444400-0.31360500 \quad-1.09552100$

C $\quad 3.13937800 \quad 0.46986500 \quad 0.05079100$

$\mathrm{H} \quad 4.08815100 \quad-0.32020700 \quad-1.71485800$

$\begin{array}{llll}0 & -0.70098900 & 2.06551500 & -0.24528100\end{array}$

$\mathrm{H}-0.00795400 \quad 0.61722900 \quad 1.97856400$

$\begin{array}{rrr}4.27838700 & 1.31375200 & 0.50111400\end{array}$

$\begin{array}{lll}4.60196100 & 1.01643000 & 1.50193400\end{array}$

$5.12942900 \quad 1.23442000 \quad-0.17668900$

$\begin{array}{lll}3.97589200 & 2.36226800 & 0.55945000\end{array}$

$\begin{array}{lll}-0.02497000 & -1.99480700 & -1.17662100\end{array}$

$\mathrm{H} \quad 0.36784900 \quad-2.71387000 \quad-1.89584800$

$\mathrm{H}-0.47007200 \quad-2.54598100 \quad-0.34521900$

$\mathrm{H}-0.82792400-1.43401200 \quad-1.66685700$

$\mathrm{N} \quad 2.18868500-1.08695700-1.47180300$

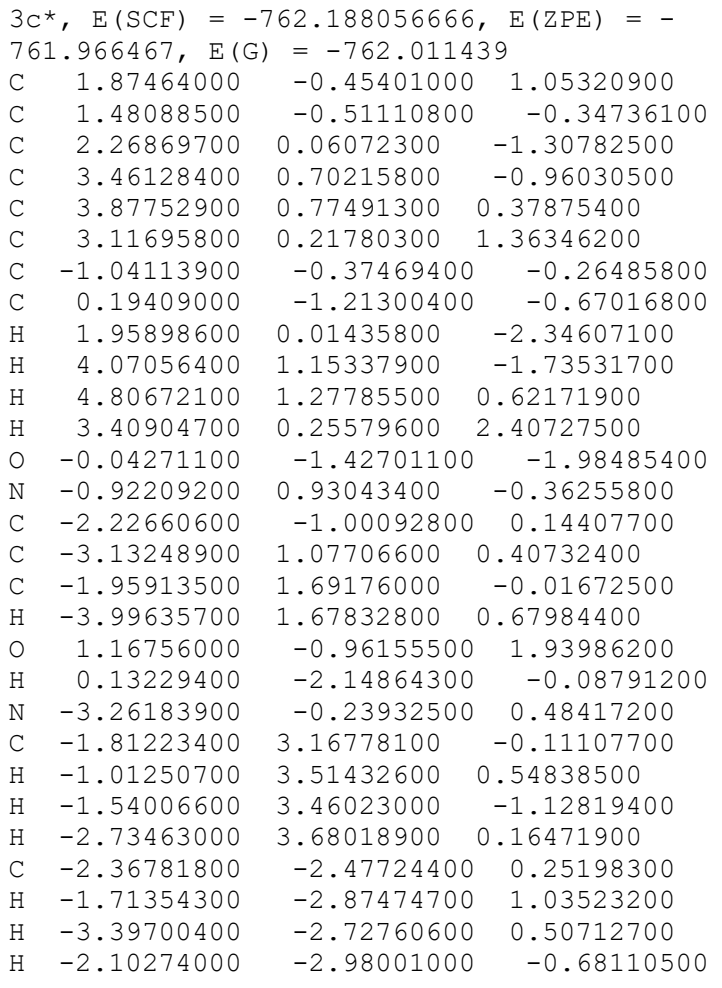

30

$3 \mathrm{C} 1 \mathrm{NH}, \mathrm{E}(\mathrm{SCF})=-762.625794302, \mathrm{E}(\mathrm{ZPE})=-$ $76 \overline{2} .389581, E(G)=-762.432373$

$\begin{array}{lrcc}\mathrm{C} & 1.68445000 & -1.18975400 & -0.20407300 \\ \mathrm{C} & 1.55524000 & 0.06217300 & 0.52757400 \\ \mathrm{C} & 2.50816800 & 1.03177800 & 0.42961900 \\ \mathrm{C} & 3.60853400 & 0.84564300 & -0.42128800 \\ \mathrm{C} & 3.75064000 & -0.33059100 & -1.17014900 \\ \mathrm{C} & 2.82429400 & -1.32847300 & -1.07795900 \\ \mathrm{C} & -0.91865000 & 0.41026700 & 0.54079100 \\ \mathrm{C} & 0.31076300 & 0.14685600 & 1.39345000 \\ \mathrm{H} & 2.42573900 & 1.94577000 & 1.00733800 \\ \mathrm{H} & 4.36142400 & 1.62183700 & -0.49251400 \\ \mathrm{H} & 4.61221500 & -0.44758500 & -1.81655200 \\ \mathrm{H} & 2.91702500 & -2.25524400 & -1.63183800 \\ \mathrm{O} & 0.17831900 & -0.97242500 & 2.13910000 \\ \mathrm{~N} & -1.73697800 & -0.63406500 & 0.38612900 \\ \mathrm{C} & -1.26535300 & 1.60368000 & -0.08923000 \\ \mathrm{C} & -3.14133000 & 0.59389700 & -0.96062700 \\ \mathrm{C} & -2.85653700 & -0.61929200 & -0.34674600 \\ \mathrm{H} & -4.03408100 & 0.68200400 & -1.57108900 \\ \mathrm{O} & 0.84423400 & -2.09249700 & -0.05957500 \\ \mathrm{H} & -1.45477300 & -1.48956800 & 0.87805400 \\ \mathrm{H} & 0.46486700 & 1.00056800 & 2.08262500 \\ \mathrm{~N} & -2.37539800 & 1.65978400 & -0.82561000 \\ \mathrm{C} & -3.67528500 & -1.84204600 & -0.44871700 \\ \mathrm{H} & -3.10764700 & -2.64431000 & -0.92715000 \\ \mathrm{H} & -3.97167200 & -2.19251900 & 0.54299100 \\ \mathrm{H} & -4.57124500 & -1.64987700 & -1.03608000 \\ \mathrm{C} & -0.44286200 & 2.83358100 & 0.03246900 \\ \mathrm{H} & 0.47189200 & 2.75377200 & -0.56208000 \\ \mathrm{H} & -1.01287500 & 3.68688300 & -0.33191900 \\ \mathrm{H} & -0.14708300 & 3.02127700 & 1.06769700 \\ & & & \end{array}$

30

$3 \mathrm{C} 44 \mathrm{NH}, \mathrm{E}(\mathrm{SCF})=-762.621776674, \mathrm{E}(\mathrm{ZPE})=-$

$76 \overline{2} .385803, E(G)=-762.429534$

C $\quad-1.96000900 \quad 0.70090500 \quad 0.62521100$

C $-1.51588600 \quad 0.00763500 \quad-0.57502100$

C $-2.17687100 \quad-1.09790300 \quad-1.02568300$

C $-3.29262200-1.58402100 \quad-0.33067500$

C $-3.75020900-0.94683300 \quad 0.83173200$

$\begin{array}{llll}C & -3.11506200 & 0.16356800 & 1.30659100\end{array}$

C $\quad 0.98260900 \quad 0.22619800 \quad-0.51963800$

C $-0.29925100 \quad 0.60043800-1.25164600$ 


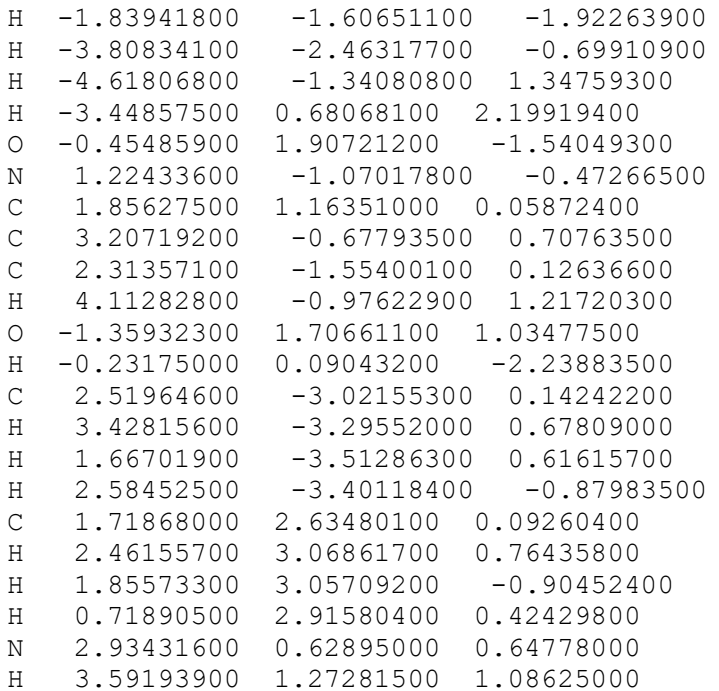

25

4a-prot_singlet, $\mathrm{E}(\mathrm{SCF})=-668.106673351$, $\mathrm{E}(\mathrm{ZPE})=-667.909127, \mathrm{E}(\mathrm{G})=-667.947327$ C $-2.38864300 \quad 0.65072500 \quad-0.16394500$ C $-1.11579100 \quad 0.10011200 \quad 0.12428400$ C $-1.03368800 \quad-1.25650500 \quad 0.49170300$ $\begin{array}{llll}C & -2.15101800 & -2.04980300 & 0.53103100\end{array}$ C $-3.39001100-1.50136300 \quad 0.19126200$ C $-3.51077900-0.17518900 \quad-0.15167900$ $\begin{array}{llll}\text { C } & 1.42864200 & 0.34726000 & 0.03895400\end{array}$ C $\quad 0.05072800 \quad 0.95183700 \quad 0.04607500$ $\mathrm{H} \quad-0.07660900 \quad-1.67643400 \quad 0.77914400$ $\mathrm{H} \quad-2.07583400 \quad-3.08810000 \quad 0.82802800$ $\mathrm{H} \quad-4.27568300 \quad-2.12642700 \quad 0.20809500$ H $\quad-4.47215000 \quad 0.26054900 \quad-0.39650200$ $\mathrm{O} \quad 0.00370200 \quad 2.17245800 \quad-0.04349500$ $\mathrm{N} \quad 1.70095200 \quad-0.66924700 \quad-0.80121900$ $\begin{array}{llll}\text { C } & 2.44556500 & 0.86561900 & 0.80610800\end{array}$ C $\quad 3.71424600 \quad 0.31453900 \quad 0.71207900$ C $3.95593200 \quad-0.72802900 \quad-0.16716800$ C $2.92043500-1.20550500-0.93350200$ $\mathrm{H} \quad 2.23462900 \quad 1.69135800 \quad 1.47300100$ $\mathrm{H} \quad 4.51883300 \quad 0.70796700 \quad 1.32123500$ $\mathrm{H} \quad 4.93928600-1.16578400 \quad-0.26824100$ $\mathrm{H} \quad 3.02210900 \quad-2.00220500-1.65769300$ $\begin{array}{llll}0 & -2.56813600 & 1.93211400 & -0.46597200\end{array}$ $\mathrm{H} \quad 0.95311800 \quad-1.03215800 \quad-1.39092500$ H $\quad-1.71260100 \quad 2.39720000 \quad-0.36309000$

25

4 a-prot triplet, $\mathrm{E}(\mathrm{SCF})=-668.044067541$, $\mathrm{E}(\mathrm{ZPE})=-667.849633, \mathrm{E}(\mathrm{G})=-667.888837$ $\begin{array}{llll}\text { C } & 2.33272900 & 0.78317100 & 0.26871900\end{array}$

C $1.14366400 \quad 0.02941200-0.13431500$

$\begin{array}{llrr}\text { C } & 1.14366400 & 0.02941200 & -0.13431500 \\ \text { C } & 1.30992900 & -1.26483400 & -0.54684500\end{array}$

C $2.56524300-1.91160900 \quad-0.46640800$

C $\quad 3.68575100-1.24389100 \quad 0.00624900$

$\begin{array}{llll}\text { C } & 3.58083500 & 0.07618900 & 0.36908500\end{array}$

C $-1.41165000 \quad 0.13901400 \quad-0.03896000$

C $-0.13407800 \quad 0.72626100 \quad-0.16875900$

H $\quad 0.47851300 \quad-1.81886500 \quad-0.96597400$

$\mathrm{H} \quad 2.64062800 \quad-2.94401400 \quad-0.78646800$

$\mathrm{H} \quad 4.63682400 \quad-1.75729200 \quad 0.07313200$

$\mathrm{H} \quad 4.43473200 \quad 0.64649400 \quad 0.71733500$

o $-0.14281900 \quad 2.04542600 \quad-0.34059400$

$\mathrm{N} \quad-2.48384000 \quad 0.95279400 \quad-0.29651300$

C $-1.70018500 \quad-1.16527100 \quad 0.39366700$

C $-3.00196000-1.58065300 \quad 0.50858700$

$\begin{array}{lrrr}\text { C } & -4.05816000 & -0.71661100 & 0.19891000\end{array}$

C $-3.76048200 \quad 0.55928500 \quad-0.19287700$

$\mathrm{H} \quad-0.89213500 \quad-1.82234100 \quad 0.68095200$

$\mathrm{H} \quad-3.21167400 \quad-2.58386700 \quad 0.85849700$

$\mathrm{H} \quad-5.08956200 \quad-1.02838800 \quad 0.28105800$

$\mathrm{H}-4.50863700 \quad 1.30263400 \quad-0.43189400$
$\begin{array}{lrrc}\mathrm{O} & 2.28169400 & 2.01267000 & 0.48557000 \\ \mathrm{H} & -2.28955000 & 1.91004300 & -0.57634000 \\ \mathrm{H} & 0.76692800 & 2.37849100 & -0.11683800\end{array}$

24

$4 \mathrm{a}$ singlet, $\mathrm{E}(\mathrm{SCF})=-667.672802233, \mathrm{E}(\mathrm{ZPE})$

$=-667.489205, \mathrm{E}(\mathrm{G})=-667.527663$

C $\quad-2.36550000 \quad 0.67983300 \quad 0.14057200$

C $-1.10466900 \quad 0.06685100 \quad-0.05712900$

C $-1.07406100 \quad-1.30418800 \quad-0.35749700$

C $-2.22656600-2.04949700 \quad-0.43210600$

C $-3.45513100-1.43472400 \quad-0.19658400$

C $-3.52638900 \quad-0.08992400 \quad 0.08677200$

C $\quad 1.47334700 \quad 0.27530300 \quad 0.01310800$

C $\quad 0.10431900 \quad 0.88531900 \quad 0.01690000$

$\mathrm{H}-0.11838800 \quad-1.77919500 \quad-0.53129000$

H $-2.17897800 \quad-3.10509300 \quad-0.66982800$

$\mathrm{H}-4.36877700 \quad-2.01720100 \quad-0.24393400$

$\mathrm{H} \quad-4.47657500 \quad 0.40349700 \quad 0.25579700$

$\begin{array}{lllll}0 & 0.04277700 & 2.11397800 & 0.08337200\end{array}$

$\begin{array}{llll}\mathrm{N} & 1.67089500 & -0.85104100 & 0.69815500\end{array}$

C $\quad 2.49335000 \quad 0.95065500 \quad-0.64510200$

$\begin{array}{llll}\text { C } & 3.76971600 & 0.42092600 & -0.60233800\end{array}$

C $\quad 3.98464300 \quad-0.74170400 \quad 0.11540300$

$\begin{array}{llll}\text { C } & 2.90542500 & -1.33450100 & 0.75241800\end{array}$

$\mathrm{H} \quad 2.27958300 \quad 1.86967600 \quad-1.17623200$

$\mathrm{H} \quad 4.58702800 \quad 0.91424900 \quad-1.11543400$

$\begin{array}{llll}\mathrm{H} & 4.96965200 & -1.18673300 & 0.18466700\end{array}$

$\mathrm{H} \quad 3.04285400 \quad-2.24330600 \quad 1.33215600$

$\begin{array}{llll}0 & -2.50460700 & 1.98236400 & 0.38540600\end{array}$

$\mathrm{H} \quad-1.60891600 \quad 2.38455200 \quad 0.32029100$

24

$4 a$ triplet, $\mathrm{E}(\mathrm{SCF})=-667.588460113, \mathrm{E}(\mathrm{ZPE})$

$=-667.407922, \mathrm{E}(\mathrm{G})=-667.447586$

C $\quad 2.33948200 \quad 0.74836600 \quad 0.26005800$

C $1.09242200 \quad 0.07322200 \quad-0.12438300$

C $1.19129900-1.23094400 \quad-0.54422500$

C $2.41085300-1.94362700 \quad-0.50615300$

C $3.58415000-1.34289300 \quad-0.07594500$

$\begin{array}{llll}\text { C } & 3.55215300 & -0.01981900 & 0.29629600\end{array}$

C $-1.46837500 \quad 0.23892100 \quad-0.02925300$

C $-0.15746200 \quad 0.81708300 \quad-0.12469000$

$\mathrm{H} \quad 0.31828200 \quad-1.74249400 \quad-0.93060300$

$\mathrm{H} \quad 2.41723900-2.97881400-0.82813400$

$\mathrm{H} \quad 4.50966200 \quad-1.90436400 \quad-0.04520300$

$\mathrm{H} \quad 4.44391500 \quad 0.50634500 \quad 0.61930800$

O $-0.11077800 \quad 2.14723300 \quad-0.26658100$

$\mathrm{N}-2.49801000 \quad 1.01090400 \quad-0.44403700$

C $-1.69864200 \quad-1.03325500 \quad 0.53029500$

$\begin{array}{llll}\text { C } & -2.98759600 & -1.50741600 & 0.62331600\end{array}$

$\begin{array}{llll}\text { C } & -4.03614200 & -0.72235800 & 0.16435800\end{array}$

C $-3.72460700 \quad 0.53018800 \quad-0.34759000$

$\mathrm{H} \quad-0.87866300 \quad-1.61629300 \quad 0.92895300$

$\mathrm{H}-3.17695500 \quad-2.47855800 \quad 1.06699600$

$\mathrm{H} \quad-5.06432200 \quad-1.05828100 \quad 0.21474300$

$\mathrm{H} \quad-4.52179800 \quad 1.18189700 \quad-0.70052700$

$\begin{array}{llll}0 & 2.36633000 & 1.97098100 & 0.52979400\end{array}$

$\mathrm{H} \quad 0.80907800 \quad 2.42371600 \quad-0.05547500$

\section{5}

$4 \mathrm{~b}$ triplet, $\mathrm{E}(\mathrm{SCF})=-750.795853760, \mathrm{E}(\mathrm{ZPE})$

$=-750.611824, \mathrm{E}(\mathrm{G})=-750.652665$

C $\quad-2.48363600 \quad 0.77134200 \quad-0.21186500$

C $-1.18715000 \quad 0.16260600 \quad 0.11927400$

C $-1.21036800 \quad-1.10788300 \quad 0.64518500$

$\begin{array}{llll}\text { C }-2.40738000 & -1.85641000 & 0.75557300\end{array}$

C $-3.62781300 \quad-1.32409700 \quad 0.38255400$

C $-3.66656400-0.02856700 \quad-0.09119400$

$\begin{array}{llll}\text { C } & 1.37082800 & 0.40169600 & 0.02647900\end{array}$

C $\quad 0.02828500 \quad 0.92249400 \quad-0.04229700$

$\mathrm{H} \quad-0.29264100 \quad-1.56865700 \quad 0.98939400$

$\mathrm{H} \quad-2.35184100 \quad-2.86554500 \quad 1.14823000$

$\mathrm{H} \quad-4.53446300 \quad-1.91023800 \quad 0.46545100$

$\mathrm{H} \quad-4.59814800 \quad 0.44797400 \quad-0.37717100$

$\begin{array}{llll}0 & -0.04036400 & 2.26316000 & -0.11692000\end{array}$

$\begin{array}{llll}\text { C } \quad 2.39228400 & 1.18911300 & 0.59173700\end{array}$ 


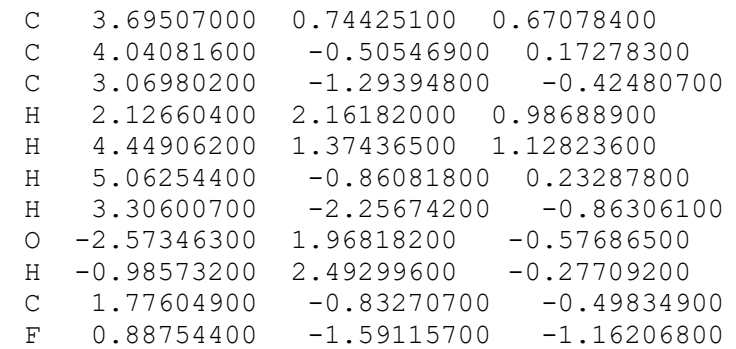

30

$4 \mathrm{C} 1 \mathrm{NH}$ singet, $\mathrm{E}(\mathrm{SCF})=-762.735910063$ $\mathrm{E}(\overline{\mathrm{Z}} \mathrm{PE})=-762.496034, \mathrm{E}(\mathrm{G})=-762$.

$\begin{array}{lrcc}\mathrm{C} & 2.77702200 & -0.34257900 & 0.45861500 \\ \mathrm{C} & 1.47233900 & -0.03072100 & 0.00120100 \\ \mathrm{C} & 1.30431700 & 1.05080000 & -0.88556600 \\ \mathrm{C} & 2.37198000 & 1.81154700 & -1.28400700 \\ \mathrm{C} & 3.64632600 & 1.50760300 & -0.79763800 \\ \mathrm{C} & 3.84977700 & 0.45155800 & 0.05906700 \\ \mathrm{C} & -1.04621700 & -0.42080700 & 0.09983200 \\ \mathrm{C} & 0.36069400 & -0.83725600 & 0.44197800 \\ \mathrm{H} & 0.31857200 & 1.27747900 & -1.27705700 \\ \mathrm{H} & 2.23241500 & 2.63439800 & -1.97336100 \\ \mathrm{H} & 4.49403800 & 2.10978100 & -1.10445400 \\ \mathrm{H} & 4.83815100 & 0.20589300 & 0.42857500 \\ \mathrm{O} & 0.46605100 & -1.86158600 & 1.10627700 \\ \mathrm{~N} & -1.51346300 & 0.73292300 & 0.60911200 \\ \mathrm{C} & -1.91630600 & -1.21012800 & -0.64049700 \\ \mathrm{C} & -3.59524000 & 0.32314300 & -0.29553200 \\ \mathrm{C} & -2.77218400 & 1.15682100 & 0.46106500 \\ \mathrm{H} & -4.62743000 & 0.61510400 & -0.46260400 \\ \mathrm{O} & 3.02960500 & -1.36290500 & 1.26997100 \\ \mathrm{H} & -0.87217700 & 1.32262400 & 1.14520900 \\ \mathrm{H} & 2.19437900 & -1.85015000 & 1.42733500 \\ \mathrm{C} & -3.20921200 & 2.41445000 & 1.09283800 \\ \mathrm{H} & -3.74804100 & 2.19589800 & 2.01952000 \\ \mathrm{H} & -2.36267300 & 3.05781800 & 1.33319500 \\ \mathrm{H} & -3.89492600 & 2.94899500 & 0.43472600 \\ \mathrm{C} & -1.49596900 & -2.49502700 & -1.25288900 \\ \mathrm{H} & -2.17103600 & -2.74249300 & -2.07102500 \\ \mathrm{H} & -0.47248700 & -2.45517600 & -1.62963200 \\ \mathrm{H} & -1.54408000 & -3.30089600 & -0.51553600 \\ \mathrm{~N} & -3.17709700 & -0.80431800 & -0.82563700\end{array}$

30

$4 \mathrm{C} 1 \mathrm{NH}$ triplet, $\mathrm{E}(\mathrm{SCF})=-762.669861466$

$-0.10834500-0.13629100$

C -1.50326300

$-2.68519900$

$-3.85207400$

$-3.86748300$

1.07116000

$-0.24237400$

$-0.63400400$

$-2.67213700$

$-4.74509300$

$-4.76183200$

$-0.39330000$

1.26974400

2.26344100

3.56101200

2.47320200

4.55849200

$-2.76912900$

0.46611000

$-1.32967900$

2.53447400

2.01984400

2.05086400

3.56960500

2.24473100

3.25187700

1.92890100

1.05946500

1.82614200

$-0.85082500$

$0.22291700 \quad 0.37884400$

$-0.42232400-0.00297000$

$-0.92862900-0.02808200$

$1.40871800-1.39743700$

$2.74758400 \quad-1.50215200$

$2.02326900-0.35701600$

$\begin{array}{ll}-0.15322600 & 0.86280100\end{array}$

$-2.23793600 \quad 0.07289700$

$0.90937000 \quad 0.27412700$

$-1.18425500-0.23877000$

$0.70466500 \quad 0.05827400$

$1.50478900 \quad 0.31809000$

$1.12888800 \quad 0.07218600$

$-1.76107400 \quad 0.93340700$

$1.47277500 \quad 0.53943600$

$\begin{array}{ll}-2.40621100 & 0.35606900\end{array}$

$2.94408900 \quad 0.64698800$

$3.53612400-0.11488200$

$3.14347700 \quad 1.60673100$

$3.27630100 \quad 0.70330600$

$-2.63228300-0.57230500$

$-2.92949800-0.85995800$

$-3.23408200 \quad 0.28221400$
$\begin{array}{llll}\mathrm{H} & 1.55358500 & -2.85467600 & -1.38716600 \\ \mathrm{~N} & 3.44125100 & -0.60356000 & -0.20913900\end{array}$

30

$4 \mathrm{C}$ 4 NH_singlet, $\mathrm{E}(\mathrm{SCF})=-762.740426219$, $\mathrm{E}(\overline{\mathrm{Z}} \mathrm{PE})=-762.500346, \mathrm{E}(\mathrm{G})=-762.542924$

C $-2.78952700 \quad-0.39984600 \quad 0.41622700$

$\begin{array}{lrrr}\text { C } & -1.48751300 & -0.00667600 & 0.02071700\end{array}$

C $-1.34423900 \quad 1.13778100 \quad-0.78459800$

C $-2.43345400 \quad 1.87350600 \quad-1.17469200$

C $-3.70740800 \quad 1.48035000 \quad-0.75938900$

$\begin{array}{llll}\text { C } & -3.88679200 & 0.36355200 & 0.02297800\end{array}$

C $\quad 1.05053200 \quad-0.35076000 \quad 0.11532500$

C $-0.35406600 \quad-0.80628900 \quad 0.42646200$

$\mathrm{H} \quad-0.35539900 \quad 1.44083200 \quad-1.10567000$

$\mathrm{H} \quad-2.30821300 \quad 2.75045200 \quad-1.79704200$

$\mathrm{H} \quad-4.57273000 \quad 2.06071600 \quad-1.05950800$

$\mathrm{H}-4.87347600 \quad 0.04969500 \quad 0.34266600$

$\begin{array}{llll}0 & -0.44858900 & -1.86181000 & 1.04726500\end{array}$

$\begin{array}{llll}\mathrm{N} & 1.43073500 & 0.81349500 & 0.59999100\end{array}$

C $1.92323500-1.20631300 \quad-0.57111000$

$\begin{array}{llll}\text { C } & 3.59858300 & 0.42328600 & -0.20777600\end{array}$

C $\quad 2.69659900 \quad 1.22212200 \quad 0.46681100$

$\mathrm{H} \quad 4.63547600 \quad 0.68255200 \quad-0.37170300$

$\begin{array}{llll}0 & -3.02193600 & -1.47814200 & 1.15876500\end{array}$

$\mathrm{H} \quad-2.16463900 \quad-1.92251600 \quad 1.32840200$

C $\quad 3.07605500 \quad 2.53152900 \quad 1.04658200$

H $2.45791800 \quad 3.32274900 \quad 0.61652400$

H $2.89065200 \quad 2.52851300 \quad 2.12301100$

$\mathrm{H} \quad 4.12529500 \quad 2.76487100 \quad 0.86895900$

C $1.56912000-2.51751600 \quad-1.15050100$

$\mathrm{H} \quad 2.34113200-2.86673400-1.83679600$

$\mathrm{H} \quad 1.44431800 \quad-3.25873500 \quad-0.35771500$

$\mathrm{H} \quad 0.62163400-2.45559400 \quad-1.68947600$

$\mathrm{N} \quad 3.17110200 \quad-0.74494400 \quad-0.70118700$

$\mathrm{H} \quad 3.84261000-1.32539500-1.20373500$

30

$4 \mathrm{C}$ 4 $\mathrm{NH}$ triplet, $\mathrm{E}(\mathrm{SCF})=-762.673396467$,

$\mathrm{E}(\overline{\mathrm{Z}} \mathrm{PE})=-762.436563, \mathrm{E}(\mathrm{G})=-762.479765$

$-0.69353600-0.25995700$

C $-1.48192100 \quad-0.05104500 \quad 0.07719000$

C $\quad-1.52697500 \quad 1.25282000 \quad 0.49990900$

C $\quad-2.73366700 \quad 1.98889000 \quad 0.53405200$

C $-3.93931200 \quad 1.41661600 \quad 0.15437400$

$\begin{array}{llll}\text { C } & -3.95549300 & 0.10489100 & -0.24327900\end{array}$

C $\quad 1.06885600 \quad-0.34658400 \quad 0.01113700$

C $-0.25745800 \quad-0.85497400 \quad 0.03078600$

$\mathrm{H} \quad-0.61603700 \quad 1.74931800 \quad 0.79967900$

$\mathrm{H} \quad-2.70177200 \quad 3.01961600 \quad 0.86674200$

$\mathrm{H} \quad-4.85242100 \quad 1.99852200 \quad 0.17294100$

$\mathrm{H}-4.87056700 \quad-0.39821500 \quad-0.53507700$

O $-0.39145000-2.16801400-0.02271400$

N $\quad 1.24608000 \quad 0.99001700 \quad-0.14954500$

C $\quad 2.20270700 \quad-1.20528300 \quad 0.14415800$

C $\quad 3.58229200 \quad 0.72519600 \quad-0.10206500$

C -2.45772900

$\begin{array}{llll}4.60231100 & 1.07622200 & -0.14085500\end{array}$

O $-2.83873800 \quad-1.91038400 \quad-0.53840000$

$\mathrm{H}-1.35388400-2.37184900-0.20121200$

C $\quad 2.56536100 \quad 2.99204400 \quad-0.38150900$

$\mathrm{H} \quad 2.06103100 \quad 3.29560200 \quad-1.30155700$

$\mathrm{H} \quad 2.06835700 \quad 3.50601700 \quad 0.44469700$

H $\quad 3.60358800 \quad 3.32072500 \quad-0.42399400$

$\begin{array}{llll}\text { C } & 2.21528500 & -2.66675600 & 0.37716000\end{array}$

H $\quad 3.23282700 \quad-3.01277600 \quad 0.56381600$

$-2.93261200 \quad 1.22915500$

$\mathrm{H} \quad 1.81469600 \quad-3.20145300 \quad-0.48523400$

$\mathrm{N} \quad 3.38457900 \quad-0.60724700 \quad 0.07684200$

$\mathrm{H} \quad 4.20950700 \quad-1.19382600 \quad 0.17802100$

29

$4 \mathrm{C}$ singlet, $\mathrm{E}(\mathrm{SCF})=-762.303930884, \mathrm{E}(\mathrm{ZPE})$

$=-762.078161, \mathrm{E}(\mathrm{G})=-762.121040$

C $\quad-1.84736100 \quad 1.09278300 \quad 0.05039600$

C $-1.58073700 \quad-0.28187400 \quad 0.22571700$

C $-2.62750800-1.19087900 \quad-0.00167800$ 


$\begin{array}{lrcc}\mathrm{C} & -3.87537700 & -0.79155700 & -0.41638400 \\ \mathrm{C} & -4.12238700 & 0.56739800 & -0.58836000 \\ \mathrm{C} & -3.12798100 & 1.48676400 & -0.35165600 \\ \mathrm{C} & 1.03697900 & -0.46364700 & 0.19849700 \\ \mathrm{C} & -0.31433800 & -0.93102400 & 0.66650900 \\ \mathrm{H} & -2.41815900 & -2.24396200 & 0.14749800 \\ \mathrm{H} & -4.65125000 & -1.52403100 & -0.60280900 \\ \mathrm{H} & -5.10081100 & 0.90875200 & -0.90830200 \\ \mathrm{H} & -3.30915600 & 2.54960600 & -0.46588400 \\ \mathrm{O} & -0.34643500 & -1.93358600 & 1.34954600 \\ \mathrm{~N} & 1.32270200 & 0.82549300 & 0.30774700 \\ \mathrm{C} & 1.98793400 & -1.37739500 & -0.28501200 \\ \mathrm{C} & 3.48121200 & 0.34987700 & -0.44675400 \\ \mathrm{C} & 2.54009500 & 1.26754600 & -0.00002000 \\ \mathrm{H} & 4.48697700 & 0.67670700 & -0.69791100 \\ \mathrm{O} & -0.99289900 & 2.08658000 & 0.29475400 \\ \mathrm{H} & -0.07357200 & 1.73881700 & 0.43807600 \\ \mathrm{C} & 2.81767900 & 2.72004500 & 0.14014700 \\ \mathrm{H} & 2.66496400 & 3.04183600 & 1.17333300 \\ \mathrm{H} & 3.84117500 & 2.95885900 & -0.14989500 \\ \mathrm{H} & 2.13472800 & 3.30255600 & -0.48439800 \\ \mathrm{C} & 1.71647500 & -2.82534200 & -0.50215400 \\ \mathrm{H} & 1.80560000 & -3.38410000 & 0.43227000 \\ \mathrm{H} & 0.71101300 & -3.00398400 & -0.88762600 \\ \mathrm{H} & 2.44670700 & -3.22087500 & -1.20856600 \\ \mathrm{~N} & 3.20134500 & -0.93410700 & -0.60427200\end{array}$

29

4c_triplet, $\mathrm{E}(\mathrm{SCF})=-762.201766518, \mathrm{E}(\mathrm{ZPE})$ $=-761.979927, \mathrm{E}(\mathrm{G})=-762.025052$

$\begin{array}{lrcc}\mathrm{C} & -1.97751800 & 0.28595200 & 0.93149400 \\ \mathrm{C} & -1.48577400 & -0.48118200 & -0.15159300 \\ \mathrm{C} & -2.30675400 & -0.70625700 & -1.26576000 \\ \mathrm{C} & -3.53411600 & -0.10185900 & -1.35483200 \\ \mathrm{C} & -3.98726400 & 0.69962900 & -0.29932500 \\ \mathrm{C} & -3.22306400 & 0.89532800 & 0.82942100 \\ \mathrm{C} & 1.06679200 & -0.42180600 & -0.08618800 \\ \mathrm{C} & -0.15580400 & -1.12468700 & -0.06386500 \\ \mathrm{H} & -1.93237000 & -1.33548200 & -2.06502500 \\ \mathrm{H} & -4.15000300 & -0.24038500 & -2.23469600 \\ \mathrm{H} & -4.96220300 & 1.16895500 & -0.36577700 \\ \mathrm{H} & -3.57837800 & 1.49903200 & 1.65613000 \\ \mathrm{O} & -0.37314100 & -2.38510800 & 0.06712600 \\ \mathrm{~N} & 0.97848900 & 0.91928600 & -0.23745500 \\ \mathrm{C} & 2.34357900 & -1.06215000 & 0.01714000 \\ \mathrm{C} & 3.33297400 & 0.99015600 & -0.18168200 \\ \mathrm{C} & 2.09794200 & 1.62473300 & -0.28315700 \\ \mathrm{H} & 4.25529400 & 1.56221100 & -0.22154900 \\ \mathrm{O} & -1.28373400 & 0.45862900 & 2.05646300 \\ \mathrm{H} & -0.42030000 & 0.02154300 & 1.98654400 \\ \mathrm{C} & 1.98420400 & 3.10094700 & -0.44961400 \\ \mathrm{H} & 1.38554300 & 3.53196500 & 0.35672100 \\ \mathrm{H} & 2.96357000 & 3.58168200 & -0.45064400 \\ \mathrm{H} & 1.47921900 & 3.34178000 & -1.38865800 \\ \mathrm{C} & 2.47285800 & -2.53542100 & 0.18408800 \\ \mathrm{H} & 2.00997400 & -2.87996500 & 1.11350700 \\ \mathrm{H} & 1.98752100 & -3.08094200 & -0.63058500 \\ \mathrm{H} & 3.52940400 & -2.80413600 & 0.20222800 \\ \mathrm{~N} & 3.43856700 & -0.33853100 & -0.03449700\end{array}$

32

ii, $\mathrm{E}(\mathrm{SCF})=-1194.36246594, \mathrm{E}(\mathrm{ZPE})=-$ $1194.143707, E(G)=-1194.197284$

$\begin{array}{llll}\mathrm{C} & 2.12356700 & -0.55863400 & -0.69649800 \\ \mathrm{C} & 2.10310400 & -1.42328800 & 0.42078400 \\ \mathrm{C} & 3.24317700 & -2.24871500 & 0.59728500 \\ \mathrm{C} & 4.28532200 & -2.20856600 & -0.30076000 \\ \mathrm{C} & 4.25592700 & -1.35874000 & -1.40482900 \\ \mathrm{C} & 3.15726900 & -0.52296500 & -1.59346200 \\ \mathrm{C} & 1.01192000 & 1.45819500 & -0.29242500 \\ \mathrm{C} & 1.00042600 & -1.44930700 & 1.29000900 \\ \mathrm{H} & 3.27546600 & -2.92626400 & 1.44327000 \\ \mathrm{H} & 5.13996800 & -2.85808100 & -0.14712600 \\ \mathrm{H} & 5.07748100 & -1.34144900 & -2.11003000 \\ \mathrm{H} & 3.10453500 & 0.16188200 & -2.43306500 \\ \mathrm{O} & 0.90742500 & -2.17264700 & 2.34408100 \\ \mathrm{~N} & -0.17265800 & 2.07339500 & -0.34539000\end{array}$

$\begin{array}{lrcc}\text { C } & 2.09003500 & 2.07865200 & 0.32201200 \\ \mathrm{C} & 1.90050000 & 3.33717400 & 0.84979800 \\ \mathrm{C} & 0.66142200 & 3.96748200 & 0.77021300 \\ \mathrm{C} & -0.36760500 & 3.29737300 & 0.16868300 \\ \mathrm{H} & 3.04648500 & 1.57670200 & 0.37428200 \\ \mathrm{H} & 2.73092600 & 3.83840800 & 1.33256300 \\ \mathrm{H} & 0.50131600 & 4.95446100 & 1.18032200 \\ \mathrm{H} & -1.37157100 & 3.69136700 & 0.07868100 \\ \mathrm{O} & 1.01638400 & 0.27024200 & -0.88647600 \\ \mathrm{H} & -1.01307800 & 1.51576000 & -0.71413900 \\ \mathrm{H} & 0.04339100 & -0.91265600 & 1.06032000 \\ \mathrm{C} & -2.50157100 & -0.13875200 & -0.09686700 \\ \mathrm{O} & -2.32290400 & 0.70060800 & -1.00249800 \\ \mathrm{O} & -1.77844700 & -0.45738000 & 0.84860900 \\ \mathrm{C} & -3.87488700 & -0.86247700 & -0.21001600 \\ \mathrm{~F} & -3.98101400 & -1.49948300 & -1.38057400 \\ \mathrm{~F} & -4.07730000 & -1.75890300 & 0.74863000 \\ \mathrm{~F} & -4.87920000 & 0.02004400 & -0.14812800\end{array}$

32

iii, $\mathrm{E}(\mathrm{SCF})=-1194.35791858, \mathrm{E}(\mathrm{ZPE})=-$ $1194.139655, E(G)=-1194.190337$

$\begin{array}{lrcc}\mathrm{C} & -0.90578700 & 1.72070400 & -0.50517000 \\ \mathrm{C} & -0.12663600 & 1.81522200 & 0.66907200 \\ \mathrm{C} & 1.23838900 & 2.15132800 & 0.49626200 \\ \mathrm{C} & 1.74704700 & 2.40255800 & -0.75729200 \\ \mathrm{C} & 0.93791600 & 2.33403000 & -1.88850200 \\ \mathrm{C} & -0.40249200 & 1.98789700 & -1.75113900 \\ \mathrm{C} & -2.65888300 & 0.14780800 & -0.29121500 \\ \mathrm{C} & -0.67917500 & 1.56872200 & 1.93316100 \\ \mathrm{H} & 1.87870900 & 2.21263400 & 1.36958200 \\ \mathrm{H} & 2.79572300 & 2.65821200 & -0.86094200 \\ \mathrm{H} & 1.34556000 & 2.53795800 & -2.87065300 \\ \mathrm{H} & -1.05832000 & 1.91465600 & -2.61154800 \\ \mathrm{O} & -0.01321100 & 1.62270000 & 3.04502400 \\ \mathrm{~N} & -1.79422500 & -0.86027500 & -0.40667400 \\ \mathrm{C} & -4.00876900 & -0.10975600 & -0.08085500 \\ \mathrm{C} & -4.42412400 & -1.41521500 & 0.01380500 \\ \mathrm{C} & -3.50471500 & -2.45876700 & -0.09834300 \\ \mathrm{C} & -2.19395600 & -2.14391100 & -0.30822300 \\ \mathrm{H} & -4.69118300 & 0.72517100 & 0.00555300 \\ \mathrm{H} & -5.47280400 & -1.63162100 & 0.17899500 \\ \mathrm{H} & -3.80935000 & -3.49291500 & -0.02200300 \\ \mathrm{H} & -1.40293800 & -2.87700100 & -0.40658900 \\ \mathrm{O} & -2.26458900 & 1.41357200 & -0.37548100 \\ \mathrm{H} & -0.72296600 & -0.74975600 & -0.58655800 \\ \mathrm{H} & -1.73973400 & 1.34070800 & 2.09231700 \\ \mathrm{C} & 1.41241700 & -1.20206500 & 0.15517000 \\ \mathrm{O} & 0.74177900 & -1.00695900 & -0.88756300 \\ \mathrm{O} & 1.04522100 & -1.39011900 & 1.30747900 \\ \mathrm{C} & 2.95307500 & -1.25817700 & -0.06723900 \\ \mathrm{~F} & 3.38529400 & -2.52269900 & 0.06268900 \\ \mathrm{~F} & 3.34542800 & -0.83452800 & -1.26687900 \\ \mathrm{~F} & 3.60899400 & -0.52854500 & 0.84040600 \\ & & & \end{array}$

32

iii*, $\mathrm{E}(\mathrm{SCF})=-1194.36153136, \mathrm{E}(\mathrm{ZPE})=-$

$1194.141276, \mathrm{E}(\mathrm{G})=-1194.194746$

$\begin{array}{llll}\mathrm{C} & 1.63574600 & -0.99860200 & -0.70762700 \\ \mathrm{C} & 1.64987400 & -1.41462900 & 0.64432000 \\ \mathrm{C} & 2.38709800 & -2.60295000 & 0.91130800 \\ \mathrm{C} & 3.01965200 & -3.28448800 & -0.10511400 \\ \mathrm{C} & 2.96447600 & -2.83984400 & -1.42730400 \\ \mathrm{C} & 2.26261100 & -1.66327600 & -1.72036500 \\ \mathrm{C} & 1.39914400 & 1.30492600 & -0.50724400 \\ \mathrm{C} & 0.96427800 & -0.68008300 & 1.63646300 \\ \mathrm{H} & 2.42821900 & -2.96523600 & 1.93280100 \\ \mathrm{H} & 3.56712600 & -4.19238900 & 0.12807800 \\ \mathrm{H} & 3.46114600 & -3.38755600 & -2.21844300 \\ \mathrm{H} & 2.20723500 & -1.27247700 & -2.73045200 \\ \mathrm{O} & 0.88100900 & -0.80242400 & 2.84909700 \\ \mathrm{~N} & 0.49186900 & 2.28898300 & -0.41864400 \\ \mathrm{C} & 2.72578600 & 1.56877800 & -0.19301000 \\ \mathrm{C} & 3.06758900 & 2.84535500 & 0.19151100 \\ \mathrm{C} & 2.10072900 & 3.85367100 & 0.26923700 \\ \mathrm{C} & 0.80971900 & 3.54684300 & -0.03454000 \\ \mathrm{H} & 3.45604700 & 0.77392600 & -0.26693800\end{array}$




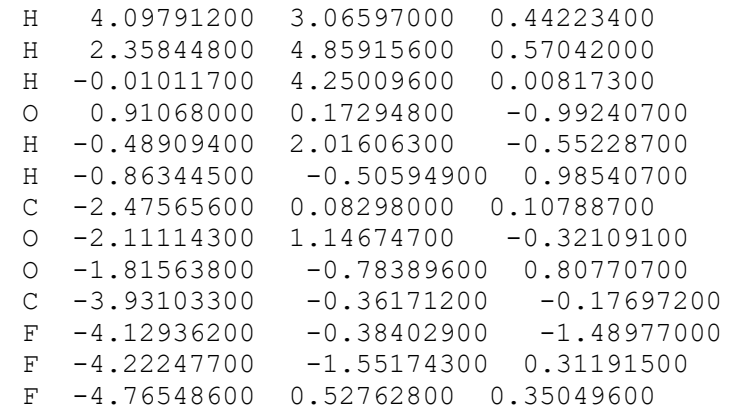

32

iv, $\mathrm{E}(\mathrm{SCF})=-1194.34588781, \mathrm{E}(\mathrm{ZPE})=-$ $1194.126066, E(G)=-1194.176067$

$\begin{array}{lrcc}\mathrm{C} & -0.44775000 & 1.81731000 & -0.58521200 \\ \mathrm{C} & -0.25065200 & 1.44817000 & 0.72863700 \\ \mathrm{C} & 0.90644800 & 1.79183900 & 1.39429100 \\ \mathrm{C} & 1.86205000 & 2.52496400 & 0.70297400 \\ \mathrm{C} & 1.64030000 & 2.90849500 & -0.61507200 \\ \mathrm{C} & 0.47768900 & 2.55644000 & -1.28807300 \\ \mathrm{C} & -2.17660200 & 0.39200100 & -0.24529000 \\ \mathrm{C} & -1.46718400 & 0.69222900 & 1.18773900 \\ \mathrm{H} & 1.06768700 & 1.47751800 & 2.41934500 \\ \mathrm{H} & 2.79005100 & 2.79598200 & 1.19232800 \\ \mathrm{H} & 2.39919900 & 3.47878900 & -1.13845600 \\ \mathrm{H} & 0.30976300 & 2.82772800 & -2.32304300 \\ \mathrm{O} & -1.37265800 & -0.32505100 & 2.02377300 \\ \mathrm{~N} & -1.74943800 & -0.91687700 & -0.66386700 \\ \mathrm{C} & -3.63725900 & 0.50453400 & -0.12454200 \\ \mathrm{C} & -4.41509500 & -0.60613100 & -0.01164300 \\ \mathrm{C} & -3.84703000 & -1.87797000 & -0.15278200 \\ \mathrm{C} & -2.49906600 & -1.97258900 & -0.52924200 \\ \mathrm{H} & -4.04075400 & 1.50721600 & -0.04126800 \\ \mathrm{H} & -5.48036800 & -0.50770100 & 0.15829400 \\ \mathrm{H} & -4.43392300 & -2.78018200 & -0.05274900 \\ \mathrm{H} & -2.03735900 & -2.92655900 & -0.76306300 \\ \mathrm{O} & -1.63053100 & 1.36094200 & -1.11703100 \\ \mathrm{H} & -0.71635300 & -1.03468000 & -0.92360800 \\ \mathrm{H} & -2.13972700 & 1.41457400 & 1.71271200 \\ \mathrm{C} & 1.37217300 & -1.31861200 & -0.04382700 \\ \mathrm{O} & 0.83375400 & -1.19330300 & -1.16869800 \\ \mathrm{O} & 0.86734000 & -1.52576300 & 1.05452100 \\ \mathrm{C} & 2.92454300 & -1.21609100 & -0.10648900 \\ \mathrm{~F} & 3.43687300 & -2.29835600 & -0.71171200 \\ \mathrm{~F} & 3.32536800 & -0.15063500 & -0.80538000 \\ \mathrm{~F} & 3.49211500 & -1.13286400 & 1.09456400 \\ & -1.90 & \end{array}$

32

$\begin{array}{lrcc}\text { I_Singlet, E (SCF) }=-1194.47061762, & E(\mathrm{ZPE}) \\ \text { C- } & -1194.248321, & \mathrm{E}(\mathrm{G})=-1194.300785 \\ \mathrm{C} & 1.98921400 & -0.65568700 & -0.61867600 \\ \mathrm{C} & 2.39734900 & -1.32342800 & 0.53451000 \\ \mathrm{C} & 3.52298700 & -2.14240100 & 0.45938000 \\ \mathrm{C} & 4.21318600 & -2.28812700 & -0.72684500 \\ \mathrm{C} & 3.78541100 & -1.61262200 & -1.86262800 \\ \mathrm{C} & 2.67145500 & -0.79156100 & -1.81178600 \\ \mathrm{C} & 0.93852300 & 1.42988400 & -0.26138100 \\ \mathrm{C} & 1.67385300 & -1.17633500 & 1.81163200 \\ \mathrm{H} & 3.83784300 & -2.66407100 & 1.35642900 \\ \mathrm{H} & 5.08388100 & -2.93120700 & -0.77365700 \\ \mathrm{H} & 4.32189700 & -1.72757600 & -2.79736100 \\ \mathrm{H} & 2.32021500 & -0.25799100 & -2.68764600 \\ \mathrm{O} & 2.03577800 & -1.68161700 & 2.84553700 \\ \mathrm{~N} & -0.23124400 & 2.04616000 & -0.26998700 \\ \mathrm{C} & 2.12927400 & 2.06773600 & 0.06393200 \\ \mathrm{C} & 2.06455000 & 3.40829100 & 0.38841500 \\ \mathrm{C} & 0.84356500 & 4.06610400 & 0.38269300 \\ \mathrm{C} & -0.27879100 & 3.34029500 & 0.04698900 \\ \mathrm{H} & 3.07065100 & 1.53390600 & 0.06145900 \\ \mathrm{H} & 2.97290800 & 3.93982300 & 0.64745500 \\ \mathrm{H} & 0.76376000 & 5.11528700 & 0.63388300 \\ \mathrm{H} & -1.26193100 & 3.79937400 & 0.02783400 \\ \mathrm{O} & 0.84765500 & 0.12217000 & -0.59836200 \\ \mathrm{H} & -1.57754000 & 1.16305400 & -0.59037700\end{array}$

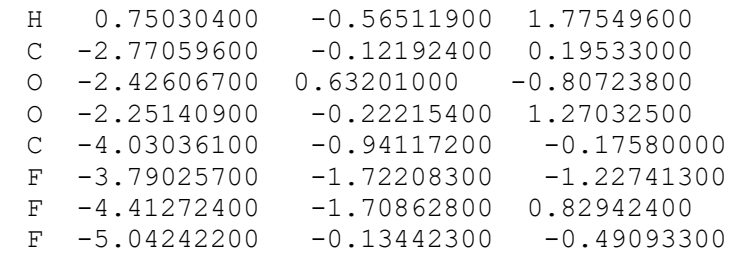

32

i_triplet, $\mathrm{E}(\mathrm{SCF})=-1194.36358222, \mathrm{E}(\mathrm{ZPE})$

$=-1194.145265, E(G)=-1194.197771$

C $\quad 1.98594000 \quad-0.62402900 \quad-0.67961300$

$\begin{array}{llll}\text { C } & 2.09114500 & -1.43240100 & 0.47678900\end{array}$

$\begin{array}{llll}\text { C } \quad 3.21378300 & -2.29242400 & 0.56023600\end{array}$

C $4.13646100-2.34053300 \quad-0.46030000$

C $3.99285400 \quad-1.54739000 \quad-1.59639200$

C $2.90556900-0.68210300-1.69518800$

$\begin{array}{llll}\text { C } & 0.99321500 & 1.47146000 & -0.27445700\end{array}$

C $1.10946500-1.36111400 \quad 1.47737800$

$\mathrm{H} \quad 3.33139600-2.92395200 \quad 1.43393800$

$\mathrm{H} \quad 4.98322700 \quad-3.01282700 \quad-0.37783300$

$\mathrm{H} \quad 4.71989400-1.59667600 \quad-2.39767000$

$\mathrm{H} \quad 2.77134500 \quad-0.04146300 \quad-2.56016400$

$\begin{array}{llll}0 & 1.12909100 & -2.03711500 & 2.57776500\end{array}$

$\mathrm{N} \quad-0.15100200 \quad 2.13508700 \quad-0.30834100$

$\begin{array}{llll}\text { C } & 2.16928200 & 2.02006400 & 0.22325000\end{array}$

$\begin{array}{llll}\text { C } & 2.12075300 & 3.31980800 & 0.68381200\end{array}$

C $\quad 0.92842500 \quad 4.02916200 \quad 0.64196800$

C $-0.18404400 \quad 3.39129500 \quad 0.14017200$

$\mathrm{H} \quad 3.08641600 \quad 1.44598600 \quad 0.24314400$

$\mathrm{H} \quad 3.01872200 \quad 3.78096700 \quad 1.07836700$

$\mathrm{H} \quad 0.86323500 \quad 5.04946800 \quad 0.99541900$

$\mathrm{H} \quad-1.14710400 \quad 3.88887700 \quad 0.09185900$

$\begin{array}{llll}0 & 0.89425900 & 0.22163000 & -0.78320700\end{array}$

$\mathrm{H} \quad-1.47173900 \quad 1.31330200 \quad-0.66995700$

$\begin{array}{llll}\mathrm{H} & 0.17997300 & -0.77816200 & 1.34710500\end{array}$

$\begin{array}{lrrr}\text { C } & -2.55665600 & -0.10158400 & 0.05597100\end{array}$

o $-2.34883900 \quad 0.79531800 \quad-0.85404000$

$\begin{array}{llll}-1.88175400 & -0.37301900 & 1.01354300\end{array}$

C $-3.88227900-0.84970100 \quad-0.22650000$

$\mathrm{F}-3.84036500-1.44466000-1.41607100$

F $\quad-4.11590900-1.77121900 \quad 0.69021000$

F $\quad-4.90638100 \quad 0.00157800 \quad-0.23203700$

24 TS_1a-2a, $\mathrm{E}(\mathrm{SCF})=-667.510972398, \mathrm{E}(\mathrm{ZPE})=$ $-6 \overline{6} 7.333083, E(G)=-667.371682$

C $1.21157900 \quad-0.66747100 \quad-0.35485100$

C $\quad 1.22051300 \quad 0.61554600 \quad 0.20173900$

$\begin{array}{llll}\text { C } & 2.41696100 & 1.18585700 & 0.62540300\end{array}$

C $\quad 3.57788500 \quad 0.44374700 \quad 0.56157600$

C $\quad 3.55073200 \quad-0.84533000 \quad 0.02651900$

C $2.37674700 \quad-1.41270100 \quad-0.43245400$

C $-1.08736600 \quad-0.50985200 \quad-0.27197200$

C $-0.10008000 \quad 1.29845800 \quad 0.13494800$

H $\quad 2.41834500 \quad 2.19772400 \quad 1.01502600$

$\mathrm{H} \quad 4.51153800 \quad 0.86116800 \quad 0.91850700$

$\mathrm{H} \quad 4.47013100 \quad-1.41715600 \quad-0.02994600$

$\mathrm{H} \quad 2.35180000-2.41210600-0.84972000$

$\begin{array}{llll}0 & -0.03048800 & 2.11814300 & -0.86798800\end{array}$

$\mathrm{N} \quad-1.37158500 \quad-0.88253400 \quad 0.99594700$

C $-2.09350600 \quad-0.13791900 \quad-1.19737300$

C $-3.32733200 \quad 0.17628500 \quad-0.69089500$

C $-3.56933200 \quad 0.01219400 \quad 0.67545300$

C $-2.56719000 \quad-0.55719700 \quad 1.45532200$

$\mathrm{H}-1.84599500-0.03158000 \quad-2.24621900$

$\mathrm{H} \quad-4.11411100 \quad 0.52748600 \quad-1.34905900$

$\mathrm{H} \quad-4.53335900 \quad 0.24487800 \quad 1.10952300$

$\mathrm{H} \quad-2.76363700 \quad-0.79810700 \quad 2.49783600$

o $0.04256100-1.14576000 \quad-0.81550400$

$\mathrm{H} \quad-0.64787400 \quad 1.61666400 \quad 1.02985900$

25

TS 1a-prot-2a-prot, $\mathrm{E}(\mathrm{SCF})=-$

$66 \overline{7} .956980789, \mathrm{E}(\mathrm{ZPE})=-667.765134, \mathrm{E}(\mathrm{G})=$ $-667.803861$ 


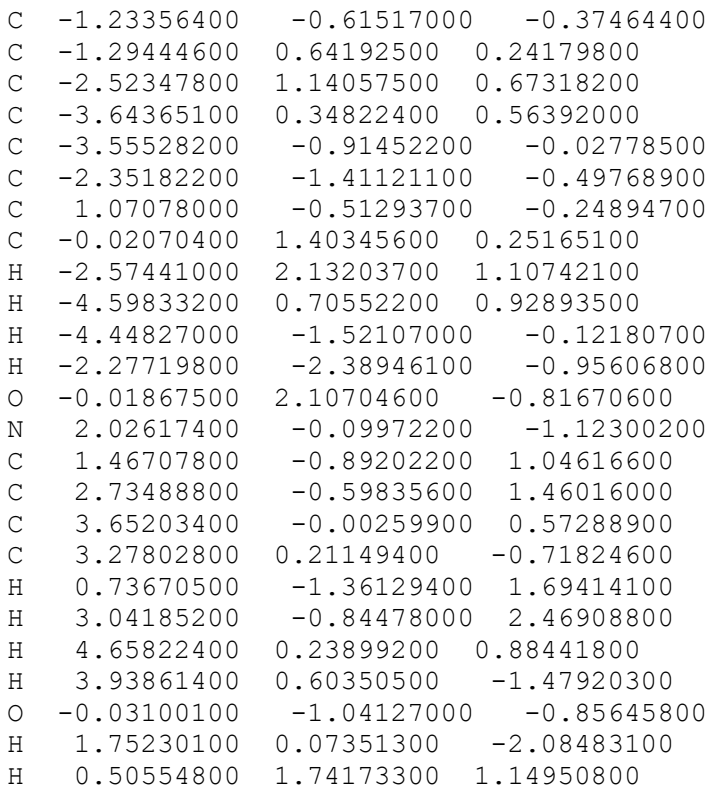

25

TS $1 \mathrm{~b}-2 \mathrm{~b}, \mathrm{E}(\mathrm{SCF})=-750.707454328, \mathrm{E}(\mathrm{ZPE})$ $-7 \overline{5} 0.526558, E(G)=-750.567289$

$\begin{array}{lrcc}\mathrm{C} & -1.30386800 & -0.40784100 & 0.60035400 \\ \mathrm{C} & -1.36700400 & 0.57875800 & -0.38258800 \\ \mathrm{C} & -2.59631400 & 0.94366100 & -0.92323700 \\ \mathrm{C} & -3.73869500 & 0.27650000 & -0.53027300 \\ \mathrm{C} & -3.65676000 & -0.72602700 & 0.43533100 \\ \mathrm{C} & -2.44825200 & -1.07813000 & 1.00521400 \\ \mathrm{C} & 1.02328300 & -0.20309800 & 0.51089600 \\ \mathrm{C} & -0.06673000 & 1.23742100 & -0.67503600 \\ \mathrm{H} & -2.63759900 & 1.73922100 & -1.65928200 \\ \mathrm{H} & -4.69701500 & 0.53431400 & -0.96465500 \\ \mathrm{H} & -4.55775700 & -1.24146100 & 0.74842900 \\ \mathrm{H} & -2.37829000 & -1.85297200 & 1.75939800 \\ \mathrm{O} & -0.07548300 & 2.42622100 & -0.14450700 \\ \mathrm{C} & 1.91732500 & 0.57273300 & 1.28125300 \\ \mathrm{C} & 3.21582100 & 0.75405100 & 0.85910900 \\ \mathrm{C} & 3.70321300 & 0.08084800 & -0.25867500 \\ \mathrm{C} & 2.87998400 & -0.81872400 & -0.94080800 \\ \mathrm{H} & 1.53035200 & 1.07381600 & 2.16103200 \\ \mathrm{H} & 3.87245100 & 1.40792600 & 1.42171000 \\ \mathrm{H} & 4.72709900 & 0.22239000 & -0.58212000 \\ \mathrm{H} & 3.24028800 & -1.39162300 & -1.78770100 \\ \mathrm{O} & -0.11318800 & -0.70469400 & 1.15980000 \\ \mathrm{H} & 0.41973300 & 1.14174400 & -1.65390200 \\ \mathrm{C} & 1.59522500 & -0.99778400 & -0.51461500 \\ \mathrm{~F} & 0.78297100 & -1.85330800 & -1.15186700\end{array}$

29

$\mathrm{TS} 1 \mathrm{C}-2 \mathrm{C}, \mathrm{E}(\mathrm{SCF})=-762.153904813, \mathrm{E}(\mathrm{ZPE})=$ $-7 \overline{6} 1.933529, E(G)=-761.976618$

$\begin{array}{lrcc}-761.933529, & & \\ \mathrm{C} & 1.51152800 & 0.05827700 & -0.72122700 \\ \mathrm{C} & 1.52720100 & -0.15241600 & 0.66056400 \\ \mathrm{C} & 2.71663800 & -0.49517400 & 1.29817400 \\ \mathrm{C} & 3.85479400 & -0.70001300 & 0.54654700 \\ \mathrm{C} & 3.81699500 & -0.51310500 & -0.83518500 \\ \mathrm{C} & 2.65313500 & -0.13711700 & -1.47978600 \\ \mathrm{C} & -0.78334400 & 0.20855700 & -0.54042500 \\ \mathrm{C} & 0.24662400 & 0.15771900 & 1.35485700 \\ \mathrm{H} & 2.72771100 & -0.61329900 & 2.37595400 \\ \mathrm{H} & 4.77845000 & -0.99749600 & 1.02785700 \\ \mathrm{H} & 4.71789300 & -0.66519100 & -1.41875000 \\ \mathrm{H} & 2.61867100 & 0.01373300 & -2.55196200 \\ \mathrm{O} & 0.41713700 & 1.27433400 & 1.99020700 \\ \mathrm{~N} & -1.21901600 & -1.05734900 & -0.59576800 \\ \mathrm{C} & -1.67026200 & 1.30535800 & -0.32380200 \\ \mathrm{C} & -3.22532600 & -0.22681400 & 0.33368200 \\ \mathrm{C} & -2.43082400 & -1.28552000 & -0.09239500 \\ \mathrm{H} & -4.21176300 & -0.41000000 & 0.74868600 \\ \mathrm{O} & 0.36220200 & 0.46824800 & -1.29435600\end{array}$

$\mathrm{H} \quad-0.36456900$

C -2.91058000

$-2.24127600$

$-3.91765200$

H -2.91810300

$-1.22826800$

$-2.00253000$

$-0.30312100$

$-1.01800000$

$-2.86245300$

$-0.61526900$

$-2.69519000$

$-3.31507300$

$-2.76702200$

$-3.11750500$

2.70076800

3.39518400

2. 91712700

2.87388900

1.05853100

1.83581000

$-0.05730900$

0.54457700

0.35559900

$-1.06566300$

$-0.56137600$

$-0.23558400$

$-0.01741400$

$-1.62088100$

29

TS_1C-2C*, E (SCF) $=-762.160934519, \mathrm{E}(\mathrm{ZPE})$

$=-761.940965, E(G)=-761.983311$

$\begin{array}{ccc}1.56971800 & 0.51899200 & -0.54218000 \\ 1.57474900 & -0.09642100 & 0.70326500 \\ 2.71885600 & -0.74615600 & 1.12951400 \\ 3.84336400 & -0.73416400 & 0.31716200 \\ 3.82047300 & -0.08353400 & -0.91293200 \\ 2.67311600 & 0.55000800 & -1.36670000 \\ -0.66377100 & 0.45828500 & -0.24476800 \\ 0.28966900 & 0.04659900 & 1.44657500 \\ 2.72803700 & -1.24601700 & 2.09203700 \\ 4.74935600 & -1.23265100 & 0.64170200 \\ 4.70806800 & -0.08311000 & -1.53507200 \\ 2.63012100 & 1.03477700 & -2.33435000 \\ -0.23445700 & -0.87404100 & 2.10262900 \\ -0.94504200 & -0.79781500 & -0.78961500 \\ -1.80717800 & 1.36132500 & 0.08231200 \\ -3.11141400 & -0.49132900 & 0.17048800 \\ -2.12848100 & -1.26232300 & -0.55690900 \\ -4.03088100 & -0.95541600 & 0.51229200 \\ 0.37502600 & 1.10588000 & -0.87315100 \\ 0.10518300 & 1.10104200 & 1.77317900 \\ -2.96220100 & 0.83417300 & 0.35195500 \\ -2.51380100 & -2.59981600 & -1.06612000 \\ -3.36689700 & -2.52407700 & -1.74674800 \\ -1.67889000 & -3.07395600 & -1.58015200 \\ -2.83297600 & -3.23539200 & -0.23396000 \\ -1.61605300 & 2.82595000 & 0.07902300 \\ -1.38489300 & 3.18089100 & -0.93091000 \\ -2.52327900 & 3.31749400 & 0.42842000 \\ -0.77229000 & 3.12270100 & 0.70897200\end{array}$

30

$\mathrm{TS} 1 \mathrm{C} 1 \mathrm{NH}-2 \mathrm{C} 1 \mathrm{NH}, \mathrm{E}(\mathrm{SCF})=-762.596671223$ $\mathrm{E}(\overline{\mathrm{Z}} \mathrm{PE})=-76 \overline{2} .362749, \mathrm{E}(\mathrm{G})=-762.405217$

$\begin{array}{llll}\mathrm{C}-1.55338400 & 0.07541500 & 0.71288900\end{array}$

C $-1.57755400 \quad-0.45608700 \quad-0.60404200$

C $-2.79721000-0.76145600 \quad-1.18817500$

C $-3.95560100 \quad-0.46926300 \quad-0.50260400$

C $-3.91166300 \quad 0.07302200 \quad 0.80489700$

C $-2.72168600 \quad 0.35316800 \quad 1.41901800$

$\begin{array}{llll}\text { C } & 0.72054800 & 0.29903100 & 0.41254900\end{array}$

C $-0.25219000 \quad-0.82465000 \quad-1.18091000$

$\mathrm{H}-2.82666800-1.18694700-2.18381300$

$\mathrm{H}-4.91813200-0.64724600 \quad-0.96666000$

$\mathrm{H} \quad-4.84218000 \quad 0.26843400 \quad 1.32393000$

$\mathrm{H} \quad-2.66511200 \quad 0.75847500 \quad 2.42120700$

O $\quad 0.00543000 \quad-2.01575100 \quad-0.91975400$

$\mathrm{N} \quad 1.71381600 \quad-0.53760400 \quad 0.85731400$

C $\quad 1.12168600 \quad 1.47576900 \quad-0.28295900$

C $\quad 3.20998000 \quad 0.54743800 \quad-0.54931700$

C $\quad 2.95131700 \quad-0.49246700 \quad 0.30728400$

$\mathrm{H} \quad 4.18455800 \quad 0.62348300 \quad-1.01631800$

$\begin{array}{lrll}\mathrm{O} & -0.36913400 & 0.32489400 & 1.27180400\end{array}$

$\mathrm{H} \quad 1.45717200 \quad-1.32277900 \quad 1.44855200$

$\mathrm{H} \quad 0.19199500 \quad-0.29842600 \quad-2.03732100$

N $\quad 2.32508300 \quad 1.54656900 \quad-0.79139100$

$\begin{array}{llll}\text { C } & 3.91997700 & -1.53937700 & 0.69178600\end{array}$

$\mathrm{H} \quad 4.86548700 \quad-1.39557300 \quad 0.17158800$

$\mathrm{H} \quad 4.10640500 \quad-1.51367300 \quad 1.76908900$

$\mathrm{H} \quad 3.52978100 \quad-2.53112400 \quad 0.44703300$

C $\quad 0.15332800 \quad 2.57498000-0.52046000$

$\mathrm{H} \quad-0.37091300 \quad 2.86000300 \quad 0.39506200$

$\mathrm{H} \quad 0.68391800 \quad 3.43921600 \quad-0.91638200$

$\mathrm{H}-0.60426300 \quad 2.27711800 \quad-1.25356900$ 
30

TS 1C 4NH-2C 4NH, E (SCF) = -762.596951506,

$\begin{array}{lrcc}\mathrm{C} & -1.53114200 & -0.11706800 & -0.72022100 \\ \mathrm{C} & -1.51483200 & 0.12988800 & 0.67271600 \\ \mathrm{C} & -2.68038600 & 0.53353900 & 1.32236500 \\ \mathrm{C} & -3.80415200 & 0.75753900 & 0.56914600 \\ \mathrm{C} & -3.80038200 & 0.51304400 & -0.82194600 \\ \mathrm{C} & -2.67432900 & 0.07551200 & -1.47890700 \\ \mathrm{C} & 0.72403000 & -0.21791100 & -0.45609300 \\ \mathrm{C} & -0.26138500 & -0.32446200 & 1.33724000 \\ \mathrm{H} & -2.67503300 & 0.69002400 & 2.39413400 \\ \mathrm{H} & -4.70978300 & 1.12079800 & 1.03876300 \\ \mathrm{H} & -4.71165600 & 0.67891400 & -1.38438700 \\ \mathrm{H} & -2.66403100 & -0.11324000 & -2.54473900 \\ \mathrm{O} & -0.52428900 & -1.49784800 & 1.73530200 \\ \mathrm{~N} & 1.09268700 & 1.09214500 & -0.51393700 \\ \mathrm{C} & 1.67425800 & -1.26355600 & -0.32000200 \\ \mathrm{C} & 3.21853600 & 0.42873200 & 0.28939600 \\ \mathrm{C} & 2.30823500 & 1.40549000 & -0.07915900 \\ \mathrm{H} & 4.22121200 & 0.62169800 & 0.63808100 \\ \mathrm{O} & -0.38115100 & -0.52985700 & -1.26129000 \\ \mathrm{H} & 0.42193500 & 0.33295000 & 1.88952900 \\ \mathrm{C} & 2.68065200 & 2.84412700 & -0.04138500 \\ \mathrm{H} & 2.63135700 & 3.26618100 & -1.04795500 \\ \mathrm{H} & 1.97289900 & 3.40004000 & 0.57703500 \\ \mathrm{H} & 3.68640400 & 2.99070300 & 0.35183700 \\ \mathrm{C} & 1.34632400 & -2.67828300 & -0.51846500 \\ \mathrm{H} & 2.18965400 & -3.32161900 & -0.27192900 \\ \mathrm{H} & 0.49241800 & -2.93959300 & 0.11760300 \\ \mathrm{H} & 1.03775200 & -2.85691700 & -1.55155100 \\ \mathrm{~N} & 2.86206500 & -0.88662200 & 0.10985200 \\ \mathrm{H} & 3.55456900 & -1.60649700 & 0.30197800 \\ & & & \end{array}$

24

TS 2a-3a, $\mathrm{E}(\mathrm{SCF})=-667.510492713, \mathrm{E}(\mathrm{ZPE})=$ $-667.331658, E(G)=-667.369844$

$\begin{array}{lrcc}\mathrm{C} & 1.06762200 & -0.68699800 & -0.40339400 \\ \mathrm{C} & 1.29342700 & 0.51457000 & 0.30329000 \\ \mathrm{C} & 2.56337900 & 0.89052800 & 0.71870500 \\ \mathrm{C} & 3.62207600 & 0.04402400 & 0.47547600 \\ \mathrm{C} & 3.41029100 & -1.14808000 & -0.23154500 \\ \mathrm{C} & 2.16504000 & -1.51773100 & -0.68714200 \\ \mathrm{C} & -1.13057800 & 0.42295000 & 0.13564600 \\ \mathrm{C} & 0.04899300 & 1.36441800 & 0.31738800 \\ \mathrm{H} & 2.70214100 & 1.83697300 & 1.23050100 \\ \mathrm{H} & 4.61849300 & 0.30038600 & 0.81408700 \\ \mathrm{H} & 4.25454900 & -1.79987500 & -0.43020000 \\ \mathrm{H} & 2.01000100 & -2.43949900 & -1.23469900 \\ \mathrm{O} & 0.33957400 & 2.13473500 & -0.75421600 \\ \mathrm{~N} & -1.50129800 & -0.22004400 & 1.29050300 \\ \mathrm{C} & -2.06581300 & 0.65164600 & -0.92201000 \\ \mathrm{C} & -3.28042300 & 0.00845200 & -0.88163900 \\ \mathrm{C} & -3.57910700 & -0.77157700 & 0.22223800 \\ \mathrm{C} & -2.65795300 & -0.81613100 & 1.29585200 \\ \mathrm{H} & -1.76699300 & 1.28775700 & -1.74593200 \\ \mathrm{H} & -3.99363000 & 0.12132200 & -1.68939000 \\ \mathrm{H} & -4.51796500 & -1.30568200 & 0.30069400 \\ \mathrm{H} & -2.92959000 & -1.35560100 & 2.20078200 \\ \mathrm{O} & -0.15616200 & -0.97914800 & -0.72108100 \\ \mathrm{H} & -0.07695200 & 1.91338700 & 1.26581500\end{array}$

25

TS 2a-prot-3a-prot, $\mathrm{E}(\mathrm{SCF})=-$

$66 \overline{7} .948882099, \mathrm{E}(\mathrm{ZPE})=-667.757759, \mathrm{E}(\mathrm{G})=$ $-667.796039$

$\begin{array}{lrcc}\mathrm{C} & -1.15243800 & -0.67976900 & -0.38373500 \\ \mathrm{C} & -1.34216500 & 0.52201400 & 0.35674700 \\ \mathrm{C} & -2.58834400 & 0.91763300 & 0.78044200 \\ \mathrm{C} & -3.66362700 & 0.09161000 & 0.49125200 \\ \mathrm{C} & -3.49243400 & -1.08480300 & -0.26770300 \\ \mathrm{C} & -2.26749900 & -1.46977700 & -0.73917400 \\ \mathrm{C} & 1.04759400 & 0.24767500 & 0.11008500 \\ \mathrm{C} & -0.05542400 & 1.31071300 & 0.33076700 \\ \mathrm{H} & -2.72544700 & 1.85722700 & 1.30215600 \\ \mathrm{H} & -4.65819900 & 0.36935800 & 0.81810500\end{array}$

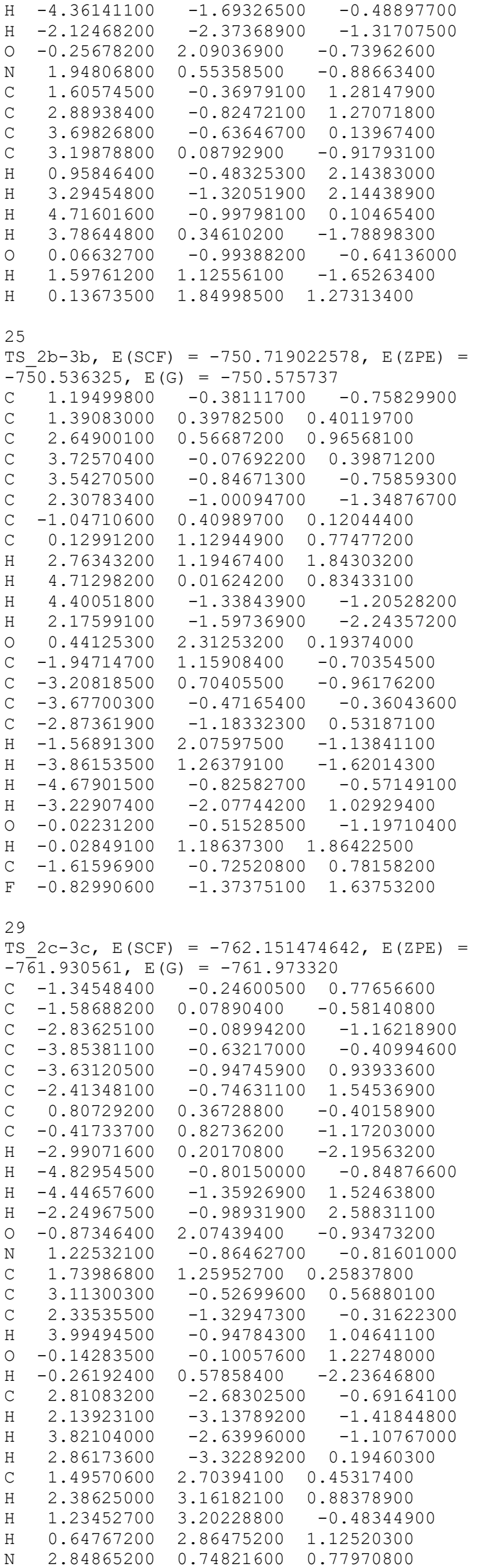


29

$\mathrm{TS} 2 \mathrm{C}^{\star}-3 \mathrm{C}^{\star}, \mathrm{E}(\mathrm{SCF})=-762.145842774, \mathrm{E}(\mathrm{ZPE})$

$=-761.925995, \mathrm{E}(\mathrm{G})=-761.969067$

$\begin{array}{lll}1.38015700 & 0.28301000 & -0.80483000\end{array}$

$1.64970300-0.48535700 \quad 0.33238300$

$2.94147000-0.79436700 \quad 0.70074000$

$3.99938600-0.32652100-0.06662900$

$3.74166600 \quad 0.45761300 \quad-1.18656000$

$2.45014700 \quad 0.78875300 \quad-1.55425200$

$\begin{array}{lll}-0.81222300 & -0.19746900 & 0.41889300\end{array}$

$0.39241900-0.90380400 \quad 1.06044100$

$3.12100600-1.39451600 \quad 1.58769600$

$5.02007600 \quad-0.55946200 \quad 0.21208400$

$\begin{array}{llll}4.56972000 & 0.82983100 & -1.78037300\end{array}$

$2.24785100 \quad 1.39480600 \quad-2.42978500$

$0.25610400 \quad-2.23187600 \quad 1.23960500$

$\begin{array}{lll}0.74430200 & -1.01730800 & -0.12831800\end{array}$

C $-1.16651300 \quad 1.14088100 \quad 0.82841300$

$\begin{array}{lll}-3.13042500 & 0.90282300 & -0.27518400\end{array}$

C $-2.86629200-0.47220200-0.51940200$

$\mathrm{H}-4.06172200 \quad 1.34267900 \quad-0.62463000$

$0.12209600 \quad 0.48681300 \quad-1.12014200$

$0.47641900 \quad-0.53877800 \quad 2.11350800$

$\begin{array}{llll}-2.31745900 & 1.66761000 & 0.42978800\end{array}$

C $-3.89210200-1.30832700-1.19281900$

$\begin{array}{llll}-4.04881100 & -0.96026900 & -2.21830400\end{array}$

$-3.57734200-2.35066600-1.21992100$

$\mathrm{H}-4.85586700-1.23345700-0.68223000$

C $\quad-0.25862700 \quad 1.96961700 \quad 1.65159200$

$\begin{array}{llll}0.74643100 & 2.02426300 & 1.21868000\end{array}$

$\mathrm{H} \quad-0.66034100 \quad 2.97776700 \quad 1.74382000$

$\mathrm{H} \quad-0.14327800 \quad 1.54828700 \quad 2.65675600$

30

TS_2C_1NH-3C_1NH, $\mathrm{E}(\mathrm{SCF})=-762.590489175$, $\mathrm{E}(\overline{\mathrm{Z}} \mathrm{PE}) \overline{\bar{C}}=-76 \overline{2} .356927, \mathrm{E}(\mathrm{G})=-762.398966$

C $-1.39421400 \quad 0.18597400 \quad 0.79559700$

C $-1.67854400 \quad-0.52296100 \quad-0.39345000$

C $-2.96451700 \quad-0.78663600 \quad-0.77630100$

C $\quad-4.00086900 \quad-0.28712800 \quad 0.01641000$

C $-3.73192900 \quad 0.46005400 \quad 1.16798400$

C $\quad-2.44185700 \quad 0.73819300 \quad 1.55577200$

C $\quad 0.73550400 \quad-0.13263800 \quad-0.36274800$

C $-0.38597100-0.91175800-1.08029800$

$\mathrm{H}-3.17662600-1.34501800-1.68084300$

$\mathrm{H}-5.02853600-0.46568600 \quad-0.27536700$

$\mathrm{H} \quad-4.55908100 \quad 0.84017500 \quad 1.75524300$

$\mathrm{H} \quad-2.21525000 \quad 1.31687100 \quad 2.44268100$

O $-0.25129500 \quad-2.25458400 \quad-0.97918200$

$\begin{array}{llll}0.79676600 & -0.89624000 & 0.01563800\end{array}$

C $\quad 1.08176800 \quad 1.23633000 \quad-0.74096700$

$\begin{array}{llll}\text { C } & 3.12643000 & 0.99313300 & 0.27284200\end{array}$

$\begin{array}{lll}2.96287600 & -0.36685200 & 0.44320900\end{array}$

$\mathrm{H} \quad 4.04398100 \quad 1.46837200 \quad 0.59900300$

$\begin{array}{llll}0 & -0.13867000 & 0.28482500 & 1.10463400\end{array}$

$\mathrm{H} \quad 1.64621100 \quad-1.90189100 \quad 0.08049600$

$\mathrm{H}-0.42435300 \quad-0.62599600 \quad-2.14685100$

$\begin{array}{llll}\mathrm{N} & 2.22369200 & 1.74867400 & -0.37039600\end{array}$

$\begin{array}{llll}3.98394400 & -1.27542100 & 0.99592800\end{array}$

$\begin{array}{llll}4.23648100 & -2.05578800 & 0.27224800\end{array}$

$\begin{array}{lll}4.88862400 & -0.72729400 & 1.25318100\end{array}$

$3.60556000-1.77373900 \quad 1.89298000$

$0.10943800 \quad 2.04990800 \quad-1.49723900$

$\mathrm{H} \quad-0.85177100 \quad 2.12736300 \quad-0.97181300$

$\mathrm{H} \quad 0.50527900 \quad 3.05252200 \quad-1.64867200$

$\mathrm{H} \quad-0.10636100 \quad 1.59995400 \quad-2.47302600$

30

TS 2C 4NH-3C 4NH, $\mathrm{E}(\mathrm{SCF})=-762.579981503$, $\mathrm{E}(\overline{\mathrm{Z}} \mathrm{PE})=-76 \overline{2} .345862, \mathrm{E}(\mathrm{G})=-762.388208$

C $-1.38604700-0.21301500 \quad 0.76078000$

C -1.62971200

C -2.87486400

C -3.88601800

C -3.66299000

C -2.44576900

$-0.21301500$

$-0.62359400$

$-0.21633500-1.17595800$

$-0.67457000-0.35074200$

$-0.85544700 \quad 1.02652500$

$-0.60052300 \quad 1.60348200$
C $\quad 0.73840000$

C -0.44504900

$\mathrm{H}-3.05352200$

$\mathrm{H} \quad-4.87123400$

$\mathrm{H} \quad-4.48168900$

H $\quad-2.26658300$

O -0.87378200

1.23386700

1.62979000

3.17715200

2. 38229600

4.13035800

$-0.16467500$

$-0.23791800$

2.86179000

3. 93465800

2.67501100

2. 32152300

1.35199700

2.21369000

1. 10223400

0.47771300

2.77850900

3.41093100

$0.29330200-0.35357100$

$0.70613900-1.23990300$

$-0.00877600-2.22450500$

$-0.86447600 \quad-0.75846500$

$-1.19848800 \quad 1.64856500$

$-0.74283500 \quad 2.66161300$

$1.97305800-1.12528200$

$-0.92128200 \quad-0.73967600$

$1.28121900 \quad 0.22971400$

$\begin{array}{ll}-0.48558300 & 0.52569800\end{array}$

$-1.33396200-0.26359800$

$-0.76479300 \quad 0.95133200$

$-0.07836100 \quad 1.15766300$

$0.36618100 \quad-2.26790300$

$-2.69893200 \quad-0.59061100$

$-2.71279200 \quad-0.78815100$

$-3.36605000 \quad 0.25641300$

$-3.08794000-1.45240300$

$2.71183100 \quad 0.40136600$

$3.22181100 \quad 0.83444800$

$3.16889500-0.56132500$

$2.86615000 \quad 1.03854300$

$0.79082700 \quad 0.67587500$

$1.43304100 \quad 1.15187400$

24

$\mathrm{TS} 3 \mathrm{a}-4 \mathrm{a}, \mathrm{E}(\mathrm{SCF})=-667.524886447, \mathrm{E}(\mathrm{ZPE})=$

$-6 \overline{6} 7.349927, E(G)=-667.390006$

C $\quad 1.91562400 \quad 0.43437600 \quad 0.83844400$

$0.18360300-0.35012500$

C $1.64088800 \quad-0.59251100-1.36307100$

C $2.90950600-1.13516600 \quad-1.20831000$

$\begin{array}{llll}\text { C } & 3.68682000 & -0.88648900 & -0.05010400\end{array}$

C $\quad 3.21865700 \quad-0.11441200 \quad 0.96936500$

C $-1.43985700 \quad 0.26783400 \quad-0.11774600$

C $-0.14954600 \quad 0.97853900 \quad-0.29693000$

$\begin{array}{lrrr}\mathrm{C} & -0.14954600 & 0.97853900 & -0.29693000 \\ \mathrm{H} & 1.05850100 & -0.79002000 & -2.25594600\end{array}$

$\mathrm{H} \quad 3.32136300 \quad-1.76809300 \quad-1.98612300$

$\mathrm{H} \quad 4.67675000 \quad-1.32254500 \quad 0.01477700$

H $\quad 3.80292600 \quad 0.09590400 \quad 1.85652100$

$\begin{array}{llll}0 & -0.04618200 & 2.07076100 & -0.97173800\end{array}$

$\mathrm{N} \quad-2.55530500 \quad 0.91506400 \quad-0.46517800$

C $-1.46894100 \quad-1.01121200 \quad 0.44513700$

C $-2.68639800 \quad-1.63109200 \quad 0.63939400$

C $-3.84427600 \quad-0.96405300 \quad 0.27128500$

$\begin{array}{llll}\text { C } & -3.71749100 & 0.30407100 & -0.27265500\end{array}$

$\mathrm{H}-0.54561500 \quad-1.50755100 \quad 0.72706100$

$\mathrm{H} \quad-2.73351100 \quad-2.62425900 \quad 1.07200400$

$\mathrm{H}-4.82227500 \quad-1.40979700 \quad 0.40471200$

$\mathrm{H} \quad-4.60542400 \quad 0.85793900 \quad-0.57003700$

$\begin{array}{llll}0 & 1.33408600 & 1.14614600 & 1.71629100\end{array}$

H $\quad 0.26699200 \quad 1.32680300 \quad 1.00874700$

25

TS 3a-prot-4a-prot, $\mathrm{E}(\mathrm{SCF})=-$

$66 \overline{7} .980709113, \mathrm{E}(\mathrm{ZPE})=-667.791872, \mathrm{E}(\mathrm{G})=$ $-667.832311$

$\begin{array}{llll}\text { C } & 1.95142700 & 0.26241300 & 0.90686700\end{array}$

C $\quad 1.18595400 \quad 0.27179400 \quad-0.31948100$

C $1.70001300-0.26582400-1.46002000$

C $2.98436600-0.82914700-1.41043300$

$\begin{array}{llll}\text { C } & 3.74128600 & -0.84741000 & -0.22024000\end{array}$

$\begin{array}{llll}\text { C } \quad 3.25473100 & -0.31320700 & 0.93587500\end{array}$

$\begin{array}{llll}\text { C } & -1.35533700 & 0.19003400 & -0.02526600\end{array}$

C $-0.11781800 \quad 1.00448400 \quad-0.10343600$

$\mathrm{H} \quad 1.14043700 \quad-0.25953600 \quad-2.38797800$

$\mathrm{H} \quad 3.40955800 \quad-1.26072200 \quad-2.30913000$

$\mathrm{H} \quad 4.72902200-1.29178500 \quad-0.23611500$

$\mathrm{H} \quad 3.81934700 \quad-0.30825900 \quad 1.85986200$

$\begin{array}{llll}0 & -0.22801300 & 2.18607500 & -0.60477700\end{array}$

$\mathrm{N}-2.48519000 \quad 0.84764700 \quad-0.33281200$

C $-1.45058600 \quad-1.13380200 \quad 0.36968300$

$\begin{array}{llll}\text { C } & -2.69448500 & -1.72987300 & 0.42996800\end{array}$

C $-3.83915300 \quad-1.01001600 \quad 0.10014500$

C $-3.70731000 \quad 0.29889500 \quad-0.28190100$

$\mathrm{H} \quad-0.55312700 \quad-1.68319600 \quad 0.62834500$

$\mathrm{H}-2.77966400 \quad-2.76600800 \quad 0.73452600$ 


$\begin{array}{lrcc}\mathrm{H} & -4.82067800 & -1.46075500 & 0.14037400 \\ \mathrm{H} & -4.53672700 & 0.93579800 & -0.55676400 \\ \mathrm{O} & 1.37296200 & 0.79048400 & 1.89968400 \\ \mathrm{H} & -2.35990100 & 1.82079700 & -0.62191700 \\ \mathrm{H} & 0.26994300 & 1.13762100 & 1.18864800 \\ & & & \\ 25 & & & \\ \mathrm{TS} & 3 \mathrm{~b}-4 \mathrm{~b}, \mathrm{E}(\mathrm{SCF}) & =-750.734915260, \mathrm{E}(\mathrm{ZPE}) \\ -750.556461, \mathrm{E}(\mathrm{G}) & =-750.597377 \\ \mathrm{C} & -2.06606600 & 0.24932200 & -0.84296300 \\ \mathrm{C} & -1.18144700 & 0.37459900 & 0.29624100 \\ \mathrm{C} & -1.57807000 & -0.08125000 & 1.52866100 \\ \mathrm{C} & -2.83852900 & -0.65410400 & 1.65035800 \\ \mathrm{C} & -3.71617500 & -0.76155600 & 0.54315900 \\ \mathrm{C} & -3.35876500 & -0.31837700 & -0.69386100 \\ \mathrm{C} & 1.37659600 & 0.43633600 & 0.00285100 \\ \mathrm{C} & 0.06532000 & 1.11804800 & -0.10022200 \\ \mathrm{H} & -0.91813900 & -0.00442500 & 2.38550200 \\ \mathrm{H} & -3.16562400 & -1.03275900 & 2.61206600 \\ \mathrm{H} & -4.69193000 & -1.20778700 & 0.69494400 \\ \mathrm{H} & -4.01643400 & -0.39035500 & -1.55112600 \\ \mathrm{O} & -0.03451300 & 2.38287700 & 0.14747100 \\ \mathrm{C} & 2.51609800 & 1.13990300 & 0.39465300 \\ \mathrm{C} & 3.75137500 & 0.52207200 & 0.47346700 \\ \mathrm{C} & 3.88084000 & -0.82278800 & 0.15721600 \\ \mathrm{C} & 2.76808300 & -1.54723400 & -0.24375000 \\ \mathrm{H} & 2.40091200 & 2.18938400 & 0.64169700 \\ \mathrm{H} & 4.61924200 & 1.09099100 & 0.78691000 \\ \mathrm{H} & 4.84580300 & -1.31192400 & 0.21986100 \\ \mathrm{H} & 2.83220200 & -2.59664400 & -0.50690100 \\ \mathrm{O} & -1.57002000 & 0.65360300 & -1.94020900 \\ \mathrm{H} & -0.45723300 & 1.00707700 & -1.42656100 \\ \mathrm{C} & 1.55302300 & -0.90597200 & -0.31675000 \\ \mathrm{~F} & 0.48375200 & -1.61437500 & -0.73431800 \\ & & & \end{array}$

29

$\mathrm{TS} 3 \mathrm{C}-4 \mathrm{C}, \mathrm{E}(\mathrm{SCF})=-762.167433842, \mathrm{E}(\mathrm{ZPE})=$ $-7 \overline{6} 1.950123, E(G)=-761.994910$

$\begin{array}{lrcc}\mathrm{C} & -2.09815200 & -0.20320600 & 0.95976900 \\ \mathrm{C} & -1.43203700 & -0.46458800 & -0.29634900 \\ \mathrm{C} & -2.04278400 & -0.13563700 & -1.48002700 \\ \mathrm{C} & -3.28021100 & 0.49592600 & -1.43634500 \\ \mathrm{C} & -3.92787600 & 0.77786600 & -0.20865000 \\ \mathrm{C} & -3.36498400 & 0.44052700 & 0.98431600 \\ \mathrm{C} & 1.09135400 & -0.38940800 & -0.06817600 \\ \mathrm{C} & -0.14232800 & -1.21516300 & -0.05597500 \\ \mathrm{H} & -1.55981900 & -0.34143400 & -2.42842500 \\ \mathrm{H} & -3.76579900 & 0.78889400 & -2.36038000 \\ \mathrm{H} & -4.89510400 & 1.26583700 & -0.23059900 \\ \mathrm{H} & -3.85420000 & 0.62715100 & 1.93237100 \\ \mathrm{O} & -0.17902200 & -2.45258800 & -0.40192700 \\ \mathrm{~N} & 0.88652900 & 0.92491500 & -0.01090600 \\ \mathrm{C} & 2.39926300 & -0.92192900 & -0.07803100 \\ \mathrm{C} & 3.21506000 & 1.21528700 & -0.03680600 \\ \mathrm{C} & 1.93382600 & 1.74542600 & 0.00731300 \\ \mathrm{H} & 4.08382800 & 1.86893500 & -0.03232400 \\ \mathrm{O} & -1.46565400 & -0.59985000 & 1.98709600 \\ \mathrm{H} & -0.47822700 & -1.11904900 & 1.30383500 \\ \mathrm{C} & 1.67456400 & 3.20951200 & 0.07495600 \\ \mathrm{H} & 1.10851500 & 3.45658800 & 0.97688800 \\ \mathrm{H} & 2.60360800 & 3.78130300 & 0.08204500 \\ \mathrm{H} & 1.07468900 & 3.53284500 & -0.77963100 \\ \mathrm{C} & 2.68942300 & -2.38271900 & -0.08418700 \\ \mathrm{H} & 2.20343000 & -2.89117700 & 0.75215500 \\ \mathrm{H} & 2.31556200 & -2.86481100 & -0.98964100 \\ \mathrm{H} & 3.76797500 & -2.53127000 & -0.01709100 \\ \mathrm{~N} & 3.43664800 & -0.09429700 & -0.07215200\end{array}$

29

$\mathrm{TS} 3 \mathrm{C}^{\star}-4 \mathrm{C}, \mathrm{E}(\mathrm{SCF})=-762.167433837, \mathrm{E}(\mathrm{ZPE})$

$=-761.950124, \mathrm{E}(\mathrm{G})=-761.994918$

C $\quad 2.09816600 \quad-0.20338400 \quad 0.95975600$

C $1.43204200-0.46455000 \quad-0.29640200$

C $2.04277000-0.13537700-1.48002900$

$\begin{array}{llll}\text { C } & 3.28018700 & 0.49619400 & -1.43624700\end{array}$

C $\quad 3.92786600 \quad 0.77791300 \quad-0.20850900$

$\begin{array}{llll}\text { C } \quad 3.36499500 & 0.44034800 & 0.98440300\end{array}$

$\begin{array}{lrcc}\mathrm{C} & -1.09135300 & -0.38939600 & -0.06822900 \\ \mathrm{C} & 0.14232900 & -1.21515300 & -0.05613900 \\ \mathrm{H} & 1.55979200 & -0.34100400 & -2.42845700 \\ \mathrm{H} & 3.76575900 & 0.78934000 & -2.36023500 \\ \mathrm{H} & 4.89509000 & 1.26589700 & -0.23038100 \\ \mathrm{H} & 3.85422300 & 0.62680400 & 1.93248500 \\ \mathrm{O} & 0.17902200 & -2.45252500 & -0.40228400 \\ \mathrm{~N} & -0.88653100 & 0.92492500 & -0.01091100 \\ \mathrm{C} & -2.39925900 & -0.92192300 & -0.07802000 \\ \mathrm{C} & -3.21506400 & 1.21528700 & -0.03667200 \\ \mathrm{C} & -1.93383200 & 1.74543100 & 0.00739200 \\ \mathrm{H} & -4.08383500 & 1.86893000 & -0.03211200 \\ \mathrm{O} & 1.46567000 & -0.60019300 & 1.98702100 \\ \mathrm{H} & 0.47822900 & -1.11926500 & 1.30368000 \\ \mathrm{~N} & -3.43664800 & -0.09429800 & -0.07204400 \\ \mathrm{C} & -1.67457100 & 3.20951700 & 0.07504300 \\ \mathrm{H} & -1.10832800 & 3.45656400 & 0.97686000 \\ \mathrm{H} & -1.07488600 & 3.53289000 & -0.77966300 \\ \mathrm{H} & -2.60361900 & 3.78129900 & 0.08234700 \\ \mathrm{C} & -2.68940700 & -2.38271500 & -0.08419800 \\ \mathrm{H} & -2.20328300 & -2.89121000 & 0.75204600 \\ \mathrm{H} & -3.76794800 & -2.53127800 & -0.01694900 \\ \mathrm{H} & -2.31568200 & -2.86476100 & -0.98973300 \\ & & & \end{array}$

30

TS_3C_1NH-4C_1NH, E (SCF) $=-762.601756280$, $\mathrm{E}(\overline{\mathrm{Z}} \mathrm{PE}) \overline{\bar{l}}=-76 \overline{2} .370392, \mathrm{E}(\mathrm{G})=-762.414207$

$\begin{array}{lrcc}\mathrm{C} & 2.21616600 & -0.45480700 & -0.88510600 \\ \mathrm{C} & 1.48831600 & -0.27588100 & 0.35398500 \\ \mathrm{C} & 2.03183700 & 0.45147500 & 1.37249200 \\ \mathrm{C} & 3.29646900 & 1.02977700 & 1.18269000 \\ \mathrm{C} & 4.01175800 & 0.86751800 & -0.02116600 \\ \mathrm{C} & 3.50218400 & 0.13632400 & -1.05314200 \\ \mathrm{C} & -1.08018400 & -0.39428300 & 0.16483800 \\ \mathrm{C} & 0.21665300 & -1.09908200 & 0.31848200 \\ \mathrm{H} & 1.50846900 & 0.58067000 & 2.31323700 \\ \mathrm{H} & 3.73880800 & 1.61222900 & 1.98260400 \\ \mathrm{H} & 4.98812100 & 1.32705300 & -0.11528500 \\ \mathrm{H} & 4.03915700 & -0.01565100 & -1.98103700 \\ \mathrm{O} & 0.25688000 & -2.19908600 & 0.98828900 \\ \mathrm{~N} & -1.08238800 & 0.94339600 & 0.04268000 \\ \mathrm{C} & -2.33230900 & -1.03754200 & 0.06883800 \\ \mathrm{C} & -3.37286900 & 1.00411100 & -0.19937800 \\ \mathrm{C} & -2.18263500 & 1.69827400 & -0.14411100 \\ \mathrm{H} & -4.30244400 & 1.54529900 & -0.33979000 \\ \mathrm{O} & 1.62795600 & -1.15424400 & -1.75747600 \\ \mathrm{H} & -0.18593300 & 1.42889800 & 0.10438500 \\ \mathrm{H} & 0.55309400 & -1.41423600 & -0.95631200 \\ \mathrm{C} & -2.03394100 & 3.16184300 & -0.26735600 \\ \mathrm{H} & -1.55244800 & 3.58016300 & 0.62037000 \\ \mathrm{H} & -1.41678500 & 3.41690900 & -1.13296300 \\ \mathrm{H} & -3.00911600 & 3.63049200 & -0.38762800 \\ \mathrm{C} & -2.47754200 & -2.51415900 & 0.11852000 \\ \mathrm{H} & -3.49430200 & -2.77518000 & -0.17232100 \\ \mathrm{H} & -1.76898700 & -3.01018400 & -0.54833800 \\ \mathrm{H} & -2.28322000 & -2.89873300 & 1.12122400 \\ \mathrm{~N} & -3.43139900 & -0.31803900 & -0.10156200\end{array}$

30

TS_3C_4NH-4C_4NH, $\mathrm{E}(\mathrm{SCF})=-762.607004779$, $\mathrm{E}(\overline{\mathrm{Z}} \mathrm{PE})=-76 \overline{2} .375502, \mathrm{E}(\mathrm{G})=-762.419020$

$\begin{array}{llll}\text { C } & -2.12672500 & -0.18393200 & 0.96514800\end{array}$

C $-1.47754400 \quad-0.49164900 \quad-0.28871400$

C $-2.08917800-0.20055900-1.47333900$

C $\quad-3.33473600 \quad 0.43383100 \quad-1.44070200$

$\begin{array}{llll}\text { C } & -3.33473600 & 0.43383100 & -1.44070200 \\ \text { C } & -3.96952200 & 0.76197800 & -0.22182300\end{array}$

C $-3.39449600 \quad 0.46551400 \quad 0.97714800$

C $1.04563200 \quad-0.38104300 \quad-0.06619200$

C $-0.17862600-1.22620800-0.03287100$

$\mathrm{H}-1.62143000 \quad-0.44335800 \quad-2.42045700$

$\mathrm{H} \quad-3.83016200 \quad 0.68371000 \quad-2.37206400$

$\mathrm{H} \quad-4.93590900 \quad 1.25052100 \quad-0.25184200$

$\mathrm{H}-3.87158900 \quad 0.69009500 \quad 1.92312000$

$0-0.14577600-2.46648500 \quad-0.36676900$

$\mathrm{N} \quad 0.86305200 \quad 0.93066300 \quad-0.02194100$

C $\quad 2.34417000 \quad-0.93996800 \quad-0.07346900$

C $3.18191500 \quad 1.28004000-0.04890700$ 


$\begin{array}{crcc}\mathrm{C} & 1.89576900 & 1.77495500 & -0.00912400 \\ \mathrm{H} & 4.07559900 & 1.88752600 & -0.05175000 \\ \mathrm{O} & -1.49066700 & -0.54662100 & 1.99776200 \\ \mathrm{H} & -0.48373500 & -1.12240000 & 1.28790600 \\ \mathrm{C} & 1.61320300 & 3.22999700 & 0.04299400 \\ \mathrm{H} & 1.01994800 & 3.52683100 & -0.82493000 \\ \mathrm{H} & 1.02365200 & 3.46406900 & 0.93223000 \\ \mathrm{H} & 2.52936900 & 3.81964300 & 0.06155700 \\ \mathrm{C} & 2.68777000 & -2.37601600 & -0.06309800 \\ \mathrm{H} & 3.76627100 & -2.51560600 & 0.02802000 \\ \mathrm{H} & 2.18900300 & -2.88144900 & 0.76569600 \\ \mathrm{H} & 2.33707900 & -2.86460400 & -0.97342100 \\ \mathrm{~N} & 3.33936800 & -0.05259300 & -0.07352400 \\ \mathrm{H} & 4.29071400 & -0.41827000 & -0.08605300\end{array}$

32

TS ii-iii*, E $(\mathrm{SCF})=-1194.35334339, \mathrm{E}(\mathrm{ZPE})$

$=-1194.138054, \mathrm{E}(\mathrm{G})=-1194.190487$

\begin{tabular}{|c|c|c|}
\hline 1.73882300 & -0.88460900 & -0.71545100 \\
\hline 1.64340800 & -1.48073500 & 0.56308300 \\
\hline 2.58055000 & -2.51793800 & 0.82847800 \\
\hline 3.46626000 & -2.93258100 & -0.13979000 \\
\hline 3.48820100 & -2.34797900 & -1.40736000 \\
\hline 2.61704700 & -1.29133500 & -1.68077300 \\
\hline 1.30017200 & 1.37663000 & -0.42923700 \\
\hline 0.68464100 & -1.06397600 & 1.51021200 \\
\hline 2.56297500 & -2.99474600 & 1.80205300 \\
\hline 4.15438800 & -3.74036200 & 0.08692300 \\
\hline 4.18163000 & -2.69172900 & -2.16461700 \\
\hline 2.63453000 & -0.77457900 & -2.63440100 \\
\hline 0.53650600 & -1.37976200 & 2.69735700 \\
\hline 0.30356100 & 2.24942800 & -0.23999900 \\
\hline 2.60296700 & 1.74907900 & -0.12697000 \\
\hline 2.82423800 & 3.02530100 & 0.33843500 \\
\hline 1.76954600 & 3.92247100 & 0.50648100 \\
\hline 0.50497100 & 3.49897000 & 0.21500900 \\
\hline 3.40907000 & 1.04122000 & -0.26478400 \\
\hline 3.83590300 & 3.33369100 & 0.57371000 \\
\hline 1.93417100 & 4.92713100 & 0.86889400 \\
\hline-0.38057500 & 4.10822400 & 0.33404500 \\
\hline 0.90693200 & 0.22329600 & -0.95348100 \\
\hline-0.66409600 & 1.87328800 & -0.36113500 \\
\hline-0.52169100 & -0.74333000 & 0.99869800 \\
\hline-2.42698200 & -0.03175100 & 0.05293700 \\
\hline-2.13668800 & 1.09149300 & -0.33339500 \\
\hline-1.74942300 & -0.89994500 & 0.66480300 \\
\hline-3.88695400 & -0.46989100 & -0.25437300 \\
\hline-4.09811800 & -0.44271500 & -1.57007400 \\
\hline-4.18005500 & -1.68413100 & 0.18086400 \\
\hline-4.74084700 & 0.38513400 & 0.30 \\
\hline
\end{tabular}

32

TS_ii-iv, $\mathrm{E}(\mathrm{SCF})=-1194.33694600, \mathrm{E}(\mathrm{ZPE})$

$=-1194.117780, \mathrm{E}(\mathrm{G})=-1194.166923$

$\begin{array}{lrcc}\text { C } & -0.62355300 & 1.86699300 & -0.47059700 \\ \text { C } & -0.31817400 & 1.46104500 & 0.81926600 \\ \text { C } & 0.91467400 & 1.79239600 & 1.36348900 \\ \text { C } & 1.80212000 & 2.55582000 & 0.62378400 \\ \text { C } & 1.45963200 & 2.98522500 & -0.65237600 \\ \text { C } & 0.24187300 & 2.64054700 & -1.21541700 \\ \text { C } & -2.37299200 & 0.33878700 & -0.46227700 \\ \text { C } & -1.35302000 & 0.65124500 & 1.49202600 \\ \text { H } & 1.17023300 & 1.44718800 & 2.35871600 \\ \text { H } & 2.76629800 & 2.8174500 & 1.04334200 \\ \text { H } & 2.15975200 & 3.58074700 & -1.22654100 \\ \text { H } & -0.02542800 & 2.93453600 & -2.22318700 \\ \text { O } & -1.09402900 & -0.46403000 & 2.07068000 \\ \text { N } & -1.79249300 & -0.84956900 & -0.79751300 \\ \text { C } & -3.74493600 & 0.34161100 & -0.14632000 \\ \text { C } & -4.38789500 & -0.84857300 & 0.04475800 \\ \text { C } & -3.70666500 & -2.06292700 & -0.14746300 \\ \text { C } & -2.40900100 & -2.02011400 & -0.57446400 \\ \text { H } & -4.23844700 & 1.29606400 & -0.01466400 \\ \text { H } & -5.43271600 & -0.85540900 & 0.33114800 \\ \text { H } & -4.19322800 & -3.01511600 & 0.00894800 \\ \text { H } & -1.81831400 & -2.90600300 & -0.77343300 \\ \mathrm{O} & -1.82387900 & 1.46553600 & -1.00793300\end{array}$

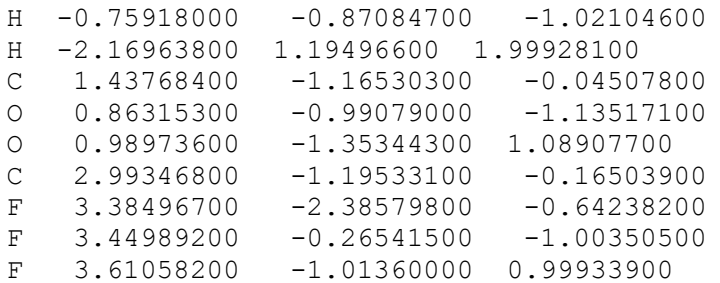

32

TS_iii*-v, E(SCF) $=-1194.36047475, E(\mathrm{ZPE})$

$=-1194.141330, \mathrm{E}(\mathrm{G})=-1194.193085$

C $-1.47909300 \quad 1.20378900 \quad-0.76006500$

C $\quad-1.57994000 \quad 1.34442400 \quad 0.63559600$

C $-2.00891100 \quad 2.58842500 \quad 1.13260800$

C $-2.30882100 \quad 3.61822500 \quad 0.25782500$

C $-2.19429800 \quad 3.44484900 \quad-1.11488700$

C $-1.78447900 \quad 2.21096600 \quad-1.63185400$

C $-1.56230100-1.11607900-0.67463800$

C $-1.19360800 \quad 0.24231900 \quad 1.45814800$

$\mathrm{H} \quad-2.08872300 \quad 2.72632200 \quad 2.20518700$

$\mathrm{H}-2.63572300 \quad 4.57398800 \quad 0.65281500$

$\mathrm{H} \quad-2.43389300 \quad 4.25629500 \quad-1.79128400$

$\mathrm{H} \quad-1.70495300 \quad 2.03943400 \quad-2.69897100$

$\begin{array}{llll}0 & -1.39153700 & -0.07343100 & 2.60546100\end{array}$

$\mathrm{N}-0.70873700-2.16628300-0.64814600$

C $-2.90050300-1.30506500 \quad-0.37665700$

C $-3.32255700 \quad-2.54595900 \quad 0.06415200$

C $-2.39813300 \quad-3.60639400 \quad 0.15127300$

C $-1.10656900-3.39936900 \quad-0.20651600$

H $-3.58120600 \quad-0.46827400 \quad-0.47026900$

$\mathrm{H} \quad-4.35556100 \quad-2.69524000 \quad 0.35011300$

$\mathrm{H} \quad-2.70370400 \quad-4.58557900 \quad 0.49436100$

$\mathrm{H}-0.33901100 \quad-4.15969700 \quad-0.17997200$

O $-1.02494700-0.01596400-1.24226500$

H $\quad 0.28235500-1.96160100-0.78275600$

$\begin{array}{llll}0.80090400 & 0.20777600 & 0.97222000\end{array}$

$\begin{array}{lll}2.42630800 & -0.27733400 & 0.09855800\end{array}$

$2.03830600-1.22929500-0.52159200$

$\begin{array}{lll}1.75454000 & 0.49181800 & 0.90549300\end{array}$

$\begin{array}{lll}3.91111100 & 0.14115200 & -0.02743000\end{array}$

$\begin{array}{llll}4.18441100 & 0.38749500 & -1.30245700\end{array}$

$\begin{array}{lll}4.21704500 & 1.21009500 & 0.68287900\end{array}$

F $\quad 4.67860400 \quad-0.86627400 \quad 0.37203400$

32

TS vi-vii, $\mathrm{E}(\mathrm{SCF})=-1194.37341671, \mathrm{E}(\mathrm{ZPE})$

$=-1194.153941, E(G)=-1194.203864$

C $-0.88568100 \quad 1.72993400 \quad-0.66461800$

C $-0.38620900 \quad 1.48225700 \quad 0.64652700$

C $\quad 0.57146100 \quad 2.33321700 \quad 1.18714100$

C $\quad 0.99348600 \quad 3.43147700 \quad 0.44040700$

C $\quad 0.47749300 \quad 3.68356200 \quad-0.82467000$

C $-0.48655700 \quad 2.84686000 \quad-1.38378100$

C $-2.02408700 \quad-0.24847100 \quad 0.27438100$

$\begin{array}{lrrr}\text { C } & -0.97318400 & 0.31187000 & 1.15848600\end{array}$

$\begin{array}{rrrr}\mathrm{C} & -0.97318400 & 0.31187000 & 1.15848600 \\ \mathrm{H} & 0.96261600 & 2.15381400 & 2.18216400\end{array}$

$\mathrm{H} \quad 1.72695900 \quad 4.10907800 \quad 0.86177500$

$\mathrm{H} \quad 0.81459200 \quad 4.55092000 \quad-1.37970500$

$\mathrm{H} \quad-0.88210200 \quad 3.01939900 \quad-2.37757300$

$\begin{array}{llll}0 & -0.59013200 & -0.40712600 & 2.20870500\end{array}$

$\mathrm{N}-1.79358100 \quad-1.55831900 \quad-0.10753200$

$\begin{array}{llll}\text { C } & -3.40598700 & 0.13663100 & 0.38742500\end{array}$

$\begin{array}{llll}\text { C } & -4.39374400 & -0.68972400 & -0.08868500\end{array}$

$\begin{array}{llll}\text { C } & -4.39374400 & -0.68972400 & -0.08868500 \\ \text { C } & -4.06565400 & -1.93061900 & -0.62481300\end{array}$

C $-2.74128100 \quad-2.34088400 \quad-0.59949100$

H $-3.61952100 \quad 1.10774600 \quad 0.81701300$

$\mathrm{H}-5.42986500-0.37816900-0.03506800$

$\mathrm{H} \quad-4.81914800 \quad-2.59635000 \quad-1.02149100$

$\begin{array}{llll}\mathrm{H} & -4.81914800 & -2.59635000 & -1.02149100 \\ \mathrm{H} & -2.42969700 & -3.31829300 & -0.94661600\end{array}$

$\begin{array}{llll}0 & -1.68303400 & 0.79486300 & -1.14458900\end{array}$

$\mathrm{H}-0.79070400 \quad-1.84611100-0.09785100$

$\begin{array}{rrrr}\mathrm{H} & -0.79070400 & -1.84611100 & -0.09785100 \\ \mathrm{H} & 0.39955400 & -0.58540900 & 2.12928700\end{array}$

C $1.84016700-1.30170700 \quad 0.39149500$

$\begin{array}{llll}\text { C } & 1.84016700 & -1.30170700 & 0.39149500 \\ 0 & 0.91944400 & -1.82273400 & -0.25141800\end{array}$

o $1.86904800 \quad-0.86274400 \quad 1.55271300$ 


$\begin{array}{cccc}\mathrm{C} & 3.16175800 & -1.09376400 & -0.39978300 \\ \mathrm{~F} & 3.31289400 & -1.95796300 & -1.39985100 \\ \mathrm{~F} & 3.15439800 & 0.13778100 & -0.93602200 \\ \mathrm{~F} & 4.24469600 & -1.18318300 & 0.36891500\end{array}$

32

TS_V_singlet-vii*, $\mathrm{E}(\mathrm{SCF})=-1194.45719021$, $\mathrm{E}(\overline{\mathrm{Z}} \mathrm{P} \overline{\mathrm{E}})=-1194.233983, \mathrm{E}(\mathrm{G})=-1194.284493$

C $-0.92197600 \quad 1.49184100 \quad 0.50126400$

C $-0.15592100 \quad 2.55076300 \quad 1.01159600$

C $-0.19777700 \quad 3.76385100 \quad 0.37990800$

C $-1.00147800 \quad 3.92158600 \quad-0.76833000$

C $-1.75758300-2.90227100-1.28865300$

C $\quad-2.02386800 \quad-0.66721100 \quad 0.22235500$

C $-1.00106000 \quad 0.16582700 \quad 0.98767000$

$\mathrm{H} \quad 0.44502400 \quad 2.39889400 \quad 1.90299800$

$\mathrm{H} \quad 0.37596300 \quad 4.60343600 \quad 0.75233800$

$\mathrm{H} \quad-1.02341400 \quad 4.89040200 \quad-1.25703300$

$H-2.36656000$

$\mathrm{O}-0.35661800 \quad-0.37740700 \quad 1.88570200$

$\mathrm{N} \quad-1.49043300 \quad-1.73894100 \quad-0.42739500$

C -3.31826000

C -4.04574200

C -3.51861600

C -2.23415400

$\mathrm{H} \quad-3.69448500$

H $\quad-5.03899500$

H $\quad-4.10161800$

$\mathrm{H} \quad-1.71805500$

O -2.36275300

H $\quad-0.50681300$

1.26087600

2. 30533000

1.42392300

2.21711100

3.78533500

3.89421300

4. 30576100

4. 49818400

0.80449000

$-0.87065100$

$-1.95672800$

$-2.93229600$

$-2.80342500$

$-0.11507900$

$-2.09301900$

$-3.78927600$

$-3.52913500$

0.59797900

$-1.68851600$

$-0.27417200$

$-0.67332200$

$-1.01293600$

$-0.27697300$

$-0.66609500$

$-1.03847400$

0.54931400

0.45217300

$-0.43071300$

$-0.83266800$

1.48344100

0.86493400

$-0.73674100$

$-1.44663500$

$-1.06947500$

$-0.68219900$

1.68667400

0.14809400

$-0.59308000$

1.38479400

$-0.30263600$

$-1.56327300$

$-1.50149500 \quad 0.44712400$

32

$\mathrm{V}, \mathrm{E}(\mathrm{SCF})=-1194.38953133, \mathrm{E}(\mathrm{ZPE})=-$

$1194.169036, E(G)=-1194.221218$

C -1.79418200

41820

$-1.02183000$

C -0.21312800

C -0.22186700

C -1.00503200

C -1.80989600

C -2.21806200

C -1.21021000

0.39801400

0.39352000

$-0.99378600$

$-2.41444300$

O -0.64201800

$\mathrm{N}-1.51008100$

C -3.40823000

C -3.48707900

C -2.52339800

C -1.53567700

$\mathrm{H}-4.13763400$

$\mathrm{H} \quad-4.29901300$

$\mathrm{H} \quad-2.53387500$

$\mathrm{H} \quad-0.78043500$

O -2.51389300

$\mathrm{H} \quad-0.69609800$

0.86101800

2.15065700

1.41041500

1.87396600

3.66822500

3.98300300

4.03647200

1. 74645900

$-0.52863600$

.38725400

2.35395200

3.64077900

0.58423600

3.9689

.19398500

0.67732100

$.96895300-0.42496900$

$3.00861000-1.05240900$

$-0.46165500 \quad-0.26718700$

$0.00192800 \quad 0.82744200$

$2.09425100 \quad 2.05145200$

$4.40539100 \quad 1.13825100$

$4.98225000 \quad-0.80931400$

$3.24916200-1.91878900$

$-0.81165500 \quad 1.60118300$

$-1.43347600-1.08655800$

$-1.04402200 \quad 0.36993400$

$-2.39337000 \quad 0.59844100$

$-3.24230600 \quad 0.05588700$

$-2.71851300 \quad-0.79167300$

$\begin{array}{ll}-0.33938900 & 0.75152700\end{array}$

$-2.80116200 \quad 1.18805800$

$\begin{array}{ll}-4.30678600 & 0.24623200\end{array}$

$-3.34469100-1.25114500$

$0.68933100-1.02577200$

$-1.06823100-1.57805100$

$-0.78076100 \quad 1.43036200$

$-0.66782700-0.00222200$

$-0.78727700 \quad-0.94257100$

$-0.69593900 \quad 1.26198600$

$-0.44231900-0.20562200$

$-0.44576400-1.48873100$

$0.72714200 \quad 0.31154300$

$-1.39681300 \quad 0.39847100$
32

$\mathrm{vi}, \mathrm{E}(\mathrm{SCF})=-1194.39391510, \mathrm{E}(\mathrm{ZPE})=-$

$1194.172473, E(G)=-1194.222895$

C $-\begin{array}{llll}0.99716700 & 1.92961700 & -0.57512200\end{array}$

C $-0.48367800 \quad 1.48963300 \quad 0.65880400$

$\begin{array}{llll}\text { C } & 0.56875700 & 2.19903600 & 1.25436500\end{array}$

C $\quad \begin{array}{llll}1.04573600 & 3.32548600 & 0.60836100\end{array}$

C $\quad 0.50616500 \quad 3.74485500 \quad-0.60656500$

C $-0.53269100 \quad 3.03916100 \quad-1.22302300$

C $-2.15308800 \quad 0.02535700 \quad-0.13283900$

C $-1.17989200 \quad 0.32627700 \quad 0.99751400$

$\mathrm{H} \quad 0.99228300 \quad 1.86303500 \quad 2.19391400$

$\mathrm{H} \quad 1.85740100 \quad 3.89093800 \quad 1.05152000$

$\mathrm{H} \quad 0.90175100 \quad 4.63053600 \quad-1.08980300$

$\mathrm{H} \quad-0.94472600 \quad 3.34841700 \quad-2.17575700$

O $-0.96369900 \quad-0.57265300 \quad 1.94499400$

$\mathrm{N}-1.69633000 \quad-1.20755200 \quad-0.77042800$

C $-3.56414600 \quad-0.06584200 \quad 0.31137900$

C $-4.26428300 \quad-1.23448300 \quad 0.28585100$

C $-3.68211000-2.38599500 \quad-0.24574000$

C $-2.37434200-2.31905800 \quad-0.75774800$

$\begin{array}{llll}\mathrm{H} & -3.98832600 & 0.85444400 & 0.69658500\end{array}$

$\mathrm{H} \quad-5.27942400 \quad-1.26693100 \quad 0.66226300$

$\mathrm{H}-4.20994000-3.32886900-0.27872200$

$\mathrm{H}-1.87901100 \quad-3.19725500 \quad-1.15789700$

o $-1.99342400 \quad 1.11041900-1.04047600$

$\mathrm{H}-0.66830300-1.22077400 \quad-0.98493600$

$\mathrm{H}-0.01175600 \quad-0.88202300 \quad 1.84086700$

$\begin{array}{lll}1.74287900 & -1.16564600 & 0.05506500\end{array}$

$1.01324700-1.17537600-0.94545400$

$1.46752600-1.19529100 \quad 1.26528500$

$3.27298700-1.08763900-0.21607000$

$3.58785100-1.17670500-1.50394800$

F $\quad 3.76211000 \quad 0.07432600 \quad 0.23398100$

F $\quad 3.92608300 \quad-2.06428600 \quad 0.42049700$

32

vii singlet, $\mathrm{E}(\mathrm{SCF})=-1194.49223519$,

$\mathrm{E}(\mathrm{Z} \overline{\mathrm{P}} \mathrm{E})=-1194.267636, \mathrm{E}(\mathrm{G})=-1194.317495$

9405600

C $\quad 1.37370700 \quad 1.78558700 \quad-0.02491400$

C $\quad 1.23552700 \quad 2.96109600 \quad-0.77268400$

C $\quad 0.10966600 \quad 3.74353000 \quad-0.66116300$

C $\quad-0.89417400 \quad 3.36868200 \quad 0.22826700$

C $-0.76202300 \quad 2.23726900 \quad 1.00562500$

$\begin{array}{llll}\text { C } & 2.55396800 & -0.52875700 & -0.13606300\end{array}$

C $\quad 2.57994400 \quad 0.97735000 \quad-0.25821300$

H $\quad 2.02602100 \quad 3.22655200 \quad-1.46535900$

$\mathrm{H} \quad 0.00307700 \quad 4.63853200 \quad-1.26181900$

$\mathrm{H} \quad-1.78702100 \quad 3.97663000 \quad 0.32360700$

$\mathrm{H} \quad-1.53054200 \quad 1.95843200 \quad 1.71865800$

O $3.62972000 \quad 1.46613500 \quad-0.61691400$

$\mathrm{N} \quad 1.44094700 \quad-1.22950700 \quad-0.40464800$

$-1.21181800 \quad 0.16571100$

C $\quad 3.70007700 \quad-2.59648500 \quad 0.21196100$

C $2.53381200-3.28426800 \quad-0.07075100$

C $1.40418500-2.56224100 \quad-0.38194300$

$\begin{array}{llll}\mathrm{H} & 4.61268500 & -0.64703300 & 0.36769500\end{array}$

$\mathrm{H} \quad 4.60455200 \quad-3.13693500 \quad 0.46361900$

$\mathrm{H} \quad 2.49107400 \quad-4.36442000 \quad-0.04903700$

H $\quad 0.44793600-3.01280700-0.61609500$

$\begin{array}{llll}0 & 0.55615600 & 0.36580700 & 1.68171000\end{array}$

$\mathrm{H} \quad 0.50917600 \quad-0.77729100 \quad-0.59452200$

$\mathrm{H} \quad-0.30768100 \quad-0.12034800 \quad 1.76144700$

C $-1.95677000 \quad-0.82888800 \quad 0.10761300$

O $-1.15893300 \quad-0.72715600 \quad-0.83759000$

$-0.86789500 \quad 1.32991600$

C $-3.46157700 \quad-0.85735100 \quad-0.27958200$

$\mathrm{F}-3.67115400-1.11009400 \quad-1.56734100$

$-1.76871700 \quad 0.41998100$

$\mathrm{F} \quad-4.00998500 \quad 0.33692400 \quad-0.01265500$

32

vii_triplet, $\mathrm{E}(\mathrm{SCF})=-1194.42605036$, $\mathrm{E}(\mathrm{Z} \overline{\mathrm{P}} \mathrm{E})=-1194.204682, \mathrm{E}(\mathrm{G})=-1194.258626$

C $-3.24602900 \quad 0.81951200 \quad-0.99870500$

C $\quad-2.47317100 \quad 0.83955900 \quad 0.24033400$ 


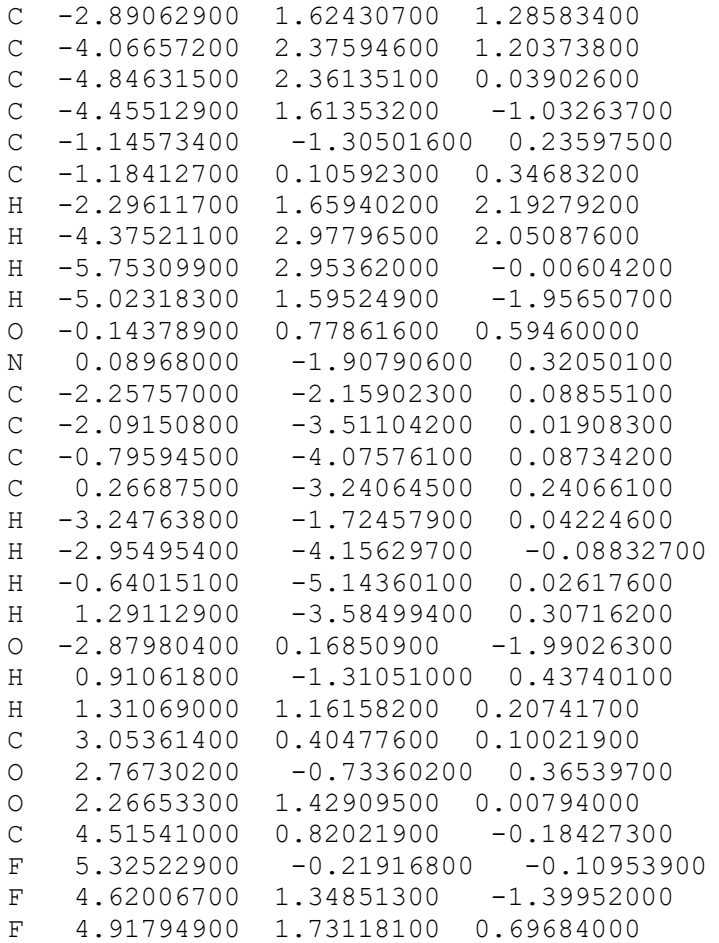

32

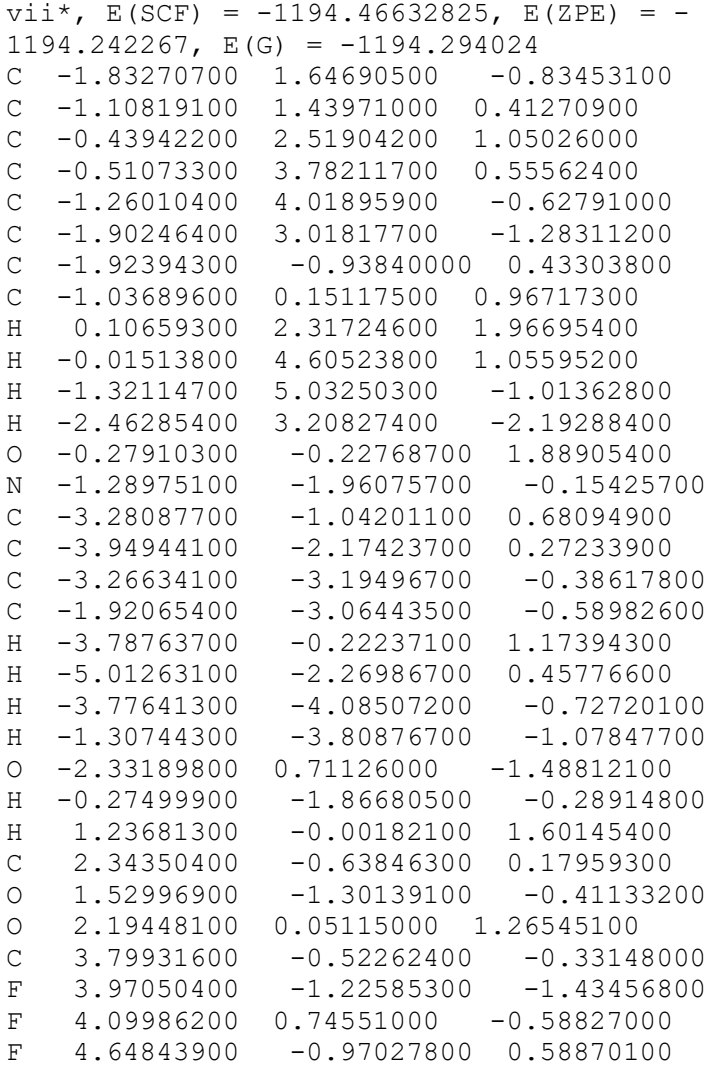

32

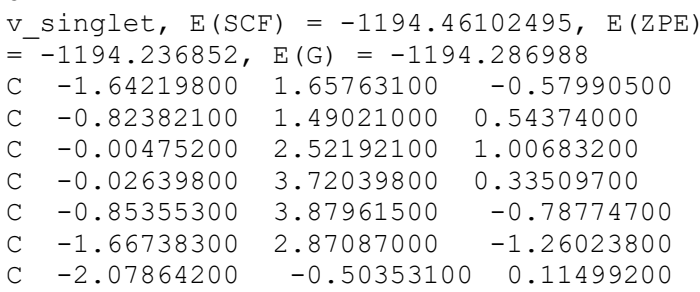

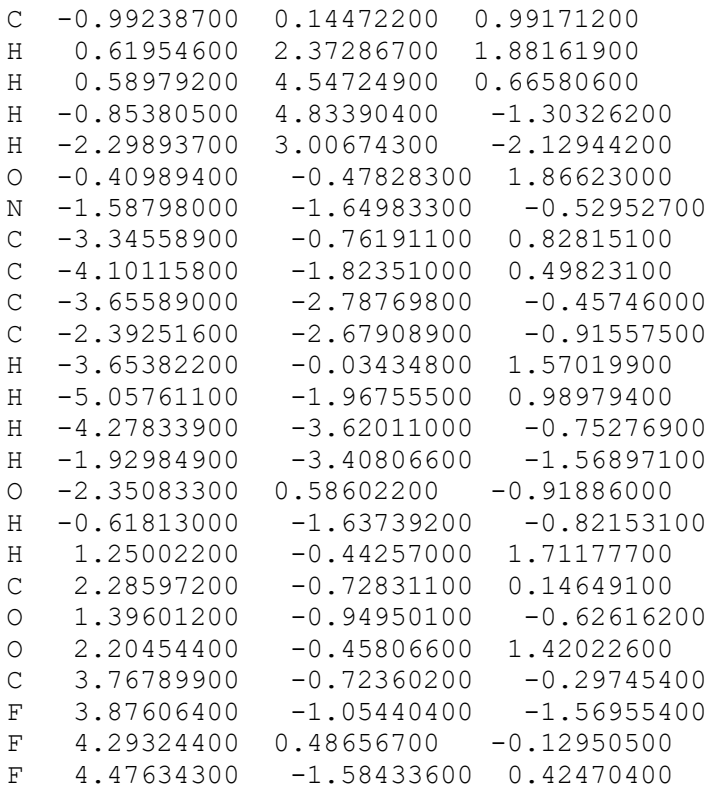

33

$\mathrm{I}, \mathrm{E}(\mathrm{SCF})=-1194.78391256, \mathrm{E}(\mathrm{ZPE})=-$

$1194.549032, \mathrm{E}(\mathrm{G})=-1194.601093$

C $-1.31189900 \quad-1.32774600 \quad 0.74707800$

C $-1.68969200-1.52337300 \quad-0.59083900$

C $-1.74984400-2.80087100 \quad-1.14244400$

C $-1.49479500 \quad-3.86397600 \quad-0.30938200$

C $-1.13255400 \quad-3.65251900 \quad 1.03129300$

C $-1.03688900 \quad-2.38598700 \quad 1.58500900$

C $-1.88873100 \quad 0.75003500 \quad 0.04422800$

C $-1.76306800 \quad-0.16959900 \quad-1.22742500$

$\mathrm{H} \quad-2.00117200 \quad-2.93909600 \quad-2.18696000$

$\mathrm{H} \quad-1.56145800 \quad-4.87692600 \quad-0.68569400$

$\mathrm{H} \quad-0.92344600 \quad-4.51258800 \quad 1.65691200$

$\mathrm{H} \quad-0.76485900 \quad-2.23103700 \quad 2.62108000$

o $-0.51350800 \quad-0.18077400 \quad-1.74951800$

$\mathrm{N} \quad-1.08331200 \quad 1.92935000 \quad-0.09434100$

C $-3.30270000 \quad 1.05073300 \quad 0.35747300$

C $-3.77443900 \quad 2.32607800 \quad 0.41592400$

C $\quad-2.90777500 \quad 3.40294700 \quad 0.21141900$

C $-1.54957300 \quad 3.14739600 \quad-0.02448700$

H $\quad-3.94747500 \quad 0.19259300 \quad 0.51760100$

$\mathrm{H} \quad-4.81894800 \quad 2.50804900 \quad 0.63638100$

$\mathrm{H} \quad-3.24828700 \quad 4.42793600 \quad 0.25474300$

$\mathrm{H} \quad-0.83152500 \quad 3.94964300 \quad-0.15010200$

O $-1.29917200 \quad-0.03012500 \quad 1.11469600$

$\mathrm{H} \quad-0.07294500 \quad 1.77178400 \quad-0.22575800$

H $\quad-2.56589000 \quad 0.01922100 \quad-1.94761700$

C $2.37891500 \quad 0.48421600 \quad-0.26005000$

o $\quad 1.73520600 \quad 1.47267200-0.02502800$

o $1.98903500-0.59121800 \quad-0.87797600$

$\mathrm{H} \quad 1.04487400 \quad-0.48605200-1.17656200$

$\begin{array}{llll}\text { C } & 3.86091900 & 0.35940500 & 0.16286200\end{array}$

F $\quad 4.24138600 \quad 1.42122400 \quad 0.84364000$

F $\quad 4.03481700 \quad-0.71756500 \quad 0.91853700$

F $\quad 4.62985900 \quad 0.24369000 \quad-0.91273000$

\section{3}

II, $\mathrm{E}(\mathrm{SCF})=-1194.79987220, \mathrm{E}(\mathrm{ZPE})=-$

$1194.565337, E(G)=-1194.614067$

C $-0.53282800 \quad 1.76425100 \quad-0.58360200$

C $-0.27971700 \quad 1.41089700 \quad 0.77183500$

C $\quad 0.87068400 \quad 1.81760700 \quad 1.37978900$

$\begin{array}{llll}\text { C } & 1.76051600 & 2.58503500 & 0.62125000\end{array}$

C $\quad 1.48337200 \quad 2.95291500 \quad-0.72430700$

C $\quad 0.33488300 \quad 2.56370400-1.34510900$

C $-2.20496600 \quad 0.28917300 \quad-0.09487800$

C $-1.44827600 \quad 0.62725100 \quad 1.27635400$

H $\quad 1.09663200 \quad 1.55141300 \quad 2.40511900$

$\mathrm{H} \quad 2.68902200 \quad 2.91978200 \quad 1.06899600$

$\mathrm{H} \quad 2.21282100 \quad 3.54910400 \quad-1.25792600$ 


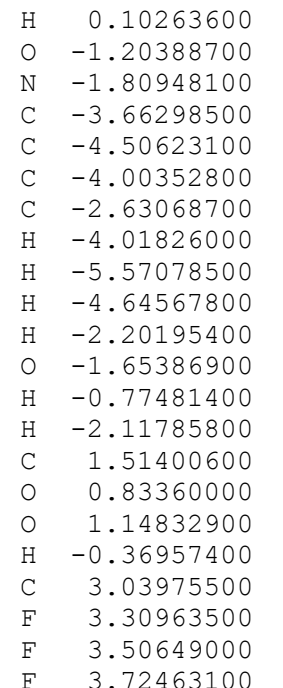

$2.81404500-2.37175700$ $-0.45012300 \quad 2.07201200$ $-0.99896800-0.57628300$ $0.45639700 \quad 0.01023000$ $-0.60725200-0.10014200$ $-1.86974200-0.42293800$ $-2.00595800 \quad-0.68544100$ $\begin{array}{lll}1.45498600 & 0.23920300\end{array}$ $-0.46864100 \quad 0.04124800$ $-2.73402700 \quad-0.51841600$ $-2.94821300-1.00740200$ $1.26639500-1.04846500$ $-1.09622600 \quad-0.76444600$ $1.31791400 \quad 1.80949500$ $-1.16793000 \quad 0.07968800$ $-0.80786000 \quad-0.90425800$ $-1.43418800 \quad 1.22722000$ $-0.90151900 \quad 1.77988300$ $-1.21964500-0.20211100$ $-1.83853900-1.34790100$ $0.03495500-0.30357300$ $\begin{array}{ll}-1.82306600 & 0.75749600\end{array}$

25

III, $\mathrm{E}(\mathrm{SCF})=-667.972206268, \mathrm{E}(\mathrm{ZPE})=$ $667.779646, \mathrm{E}(\mathrm{G})=-667.818446$
C -1.14908200
$-0.62534800$
$-1.32395100$
$-2.56265000$
$-3.63925900$
$-3.46448000$
$-2.23201900$
1.00015200
$-0.00731700$
$-2.72635400$
$-4.63815900$
$-4.33252200$
$-2.06189000$
$-0.01993300$
1.90907200
1.51751400
2.81708800
3.64963800
3.13213300
0.82531900
3.20325500
4.68380700
3.80442500
0.08056100
0.73880900
.5425990
$-0.42033300$
$0.87943200 \quad 0.82924800$
$0.05753600 \quad 0.45784700$
$-1.08461900-0.36024300$
$-1.44506900 \quad-0.81519600$
$0.14403000 \quad 0.04560300$
$1.25369800 \quad 0.44732300$
$1.76075200 \quad 1.43695600$
$0.30677800 \quad 0.79679700$
$-1.67856600-0.61755100$
$-2.31758800-1.43263600$
$2.30028500-0.47598500$
$0.61263500-0.92246700$
$-0.64414400 \quad 1.18074500$
$-1.05099600 \quad 1.18232100$
$-0.66814300 \quad 0.13070600$
$0.19973600-0.87141200$
$-0.89873500 \quad 1.97779900$
$-1.65176200 \quad 1.99682000$
$-0.98307900 \quad 0.08211200$
$0.56891200-1.64272300$
$-0.86442600-0.73050000$
$2.15266700-1.07132800$
$1.61303400 \quad 1.45559700$

33

II $I^{*} \mathrm{E}(\mathrm{SCF})=-1194.79920571, \mathrm{E}(\mathrm{ZPE})=-$

$1194.565107, E(G)=-1194.613872$

$\begin{array}{lrcc}\mathrm{C} & -0.72247000 & 1.83770800 & -0.59183400 \\ \mathrm{C} & -0.42474700 & 1.47226400 & 0.75492200 \\ \mathrm{C} & 0.73507700 & 1.89054300 & 1.33633200 \\ \mathrm{C} & 1.58096100 & 2.70408900 & 0.57300100 \\ \mathrm{C} & 1.25831300 & 3.09221900 & -0.75485400 \\ \mathrm{C} & 0.10395700 & 2.68462000 & -1.35411300 \\ \mathrm{C} & -2.25903100 & 0.21499000 & -0.09092200 \\ \mathrm{C} & -1.57404300 & 0.66810900 & 1.26596700 \\ \mathrm{H} & 0.99162000 & 1.61727400 & 2.35296100 \\ \mathrm{H} & 2.50827300 & 3.06086300 & 1.00611800 \\ \mathrm{H} & 1.95515300 & 3.72186800 & -1.29371400 \\ \mathrm{H} & -0.15961800 & 2.94839500 & -2.37008400 \\ \mathrm{O} & -1.30415500 & -0.35344600 & 2.13730600 \\ \mathrm{~N} & -1.63007900 & -0.97674700 & -0.55049500 \\ \mathrm{C} & -3.72917000 & 0.21674400 & -0.02142900 \\ \mathrm{C} & -4.41787100 & -0.95046900 & -0.13647200 \\ \mathrm{C} & -3.72868000 & -2.12970600 & -0.42724100 \\ \mathrm{C} & -2.33315100 & -2.05935100 & -0.65916400 \\ \mathrm{H} & -4.21850300 & 1.15975000 & 0.19724500 \\ \mathrm{H} & -5.49442200 & -0.96160600 & -0.01903600 \\ \mathrm{H} & -4.23892800 & -3.07775700 & -0.52989500 \\ \mathrm{H} & -1.80040400 & -2.95380700 & -0.97447600\end{array}$

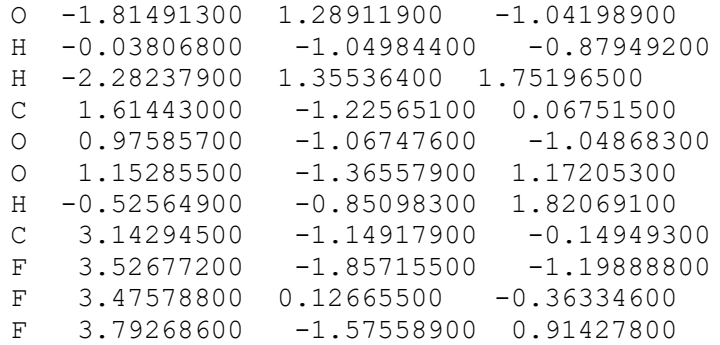

25

$\mathrm{IV}, \mathrm{E}(\mathrm{SCF})=-667.979360620, \mathrm{E}(\mathrm{ZPE})=-$

$667.788147, E(G)=-667.828313$

$\begin{array}{lrcc}\mathrm{C} & -1.15648000 & -0.72480900 & -0.50782600 \\ \mathrm{C} & -1.24550000 & 0.48826500 & 0.28915100 \\ \mathrm{C} & -2.44172400 & 0.87411300 & 0.81187000 \\ \mathrm{C} & -3.60061000 & 0.12908600 & 0.54765600 \\ \mathrm{C} & -3.55165200 & -1.01924500 & -0.25790100 \\ \mathrm{C} & -2.37327700 & -1.44622100 & -0.79049000 \\ \mathrm{C} & 1.23957500 & 0.50456700 & 0.26437700 \\ \mathrm{C} & 0.00434500 & 1.33767300 & 0.35488500 \\ \mathrm{H} & -2.50268600 & 1.77117900 & 1.41829600 \\ \mathrm{H} & -4.54679400 & 0.45001900 & 0.96653400 \\ \mathrm{H} & -4.46221700 & -1.57465700 & -0.44867100 \\ \mathrm{H} & -2.29883100 & -2.33518300 & -1.40452000 \\ \mathrm{O} & -0.05286800 & 2.31009700 & -0.63697200 \\ \mathrm{~N} & 2.11431000 & 0.84975500 & -0.68644000 \\ \mathrm{C} & 1.50220700 & -0.51046800 & 1.22129000 \\ \mathrm{C} & 2.67733000 & -1.22732800 & 1.11950800 \\ \mathrm{C} & 3.54936300 & -0.90422800 & 0.10140800 \\ \mathrm{C} & 3.22602600 & 0.16013200 & -0.77460500 \\ \mathrm{H} & 0.76984300 & -0.71513800 & 1.99547900 \\ \mathrm{H} & 2.90610900 & -2.01957100 & 1.82043900 \\ \mathrm{H} & 4.48456500 & -1.43340400 & -0.03131200 \\ \mathrm{H} & 3.92376100 & 0.44241200 & -1.55713900 \\ \mathrm{O} & -0.05077100 & -1.12753500 & -0.91585300 \\ \mathrm{H} & 0.76322300 & 2.22664500 & -1.16250100 \\ \mathrm{H} & 0.01434800 & 1.80970100 & 1.35514800 \\ & & & \end{array}$

33

TS I-II, E $(\mathrm{SCF})=-1194.77990104, \mathrm{E}(\mathrm{ZPE})=$ $-1 \overline{1} 94.549732, E(G)=-1194.598883$

C -1.41150400

C $-1.33405200 \quad 2.94144600 \quad 1.10990200$

C $-\begin{array}{llll}0.84621300 & 3.92655600 & 0.28288300\end{array}$

C $\quad-0.39330900 \quad 3.63735300 \quad-1.03622300$

C $-0.44823000 \quad 2.37630100 \quad-1.56780700$

C $\begin{array}{llll}-1.89216800 & -0.57318400 & -0.02990400\end{array}$

C $-1.66175600 \quad 0.33800300 \quad 1.24250700$

$\mathrm{H} \quad-1.65532000 \quad 3.15408700 \quad 2.12157200$

$\mathrm{H} \quad-0.79500000 \quad 4.95043500 \quad 0.63234700$

$\mathrm{H} \quad-0.00009500 \quad 4.45065300 \quad-1.63447600$

$\mathrm{H} \quad-0.11079900 \quad 2.14769200 \quad-2.57010500$

$\begin{array}{llll}0 & -0.48050000 & 0.09509800 & 1.85507800\end{array}$

$\mathrm{N} \quad-1.28130900 \quad-1.85178100 \quad 0.12130000$

C $-3.31100000-0.64083200 \quad-0.41960500$

C $-3.96941800-1.82712400 \quad-0.53531900$

C $-3.28642000-3.02665300-0.32579200$

C $\quad-1.92261200 \quad-2.98329000 \quad-0.00513100$

$\mathrm{H} \quad-3.80806900 \quad 0.31014500 \quad-0.58183500$

$\mathrm{H} \quad-5.01872200 \quad-1.84017200 \quad-0.80254000$

$\mathrm{H} \quad-3.77355800 \quad-3.98722100 \quad-0.41723800$

$\mathrm{H}-1.34479200-3.88713300 \quad 0.14950400$

$\begin{array}{llll}0 & -1.13534400 & 0.12820400 & -1.09022200\end{array}$

$\mathrm{H}-0.26528000 \quad-1.85135000 \quad 0.33385800$

$\mathrm{H} \quad-2.53157900 \quad 0.27041400 \quad 1.90540500$

C $\quad 2.13586800 \quad-0.75633400 \quad 0.35382500$

o $1.51171700 \quad-1.78495100 \quad 0.19251100$

$\begin{array}{llll}0 & 1.75021700 & 0.32819800 & 0.91210200\end{array}$

H $\quad 0.72544700 \quad 0.23725900 \quad 1.30577700$

C $\quad 3.58834600-0.64242000 \quad-0.16602600$

F $\quad 4.00580500 \quad-1.78013100 \quad-0.68963500$

F $\quad 3.65764800 \quad 0.30047000-1.10524100$

F $\quad 4.41532200 \quad-0.30357000 \quad 0.81673100$ 
33

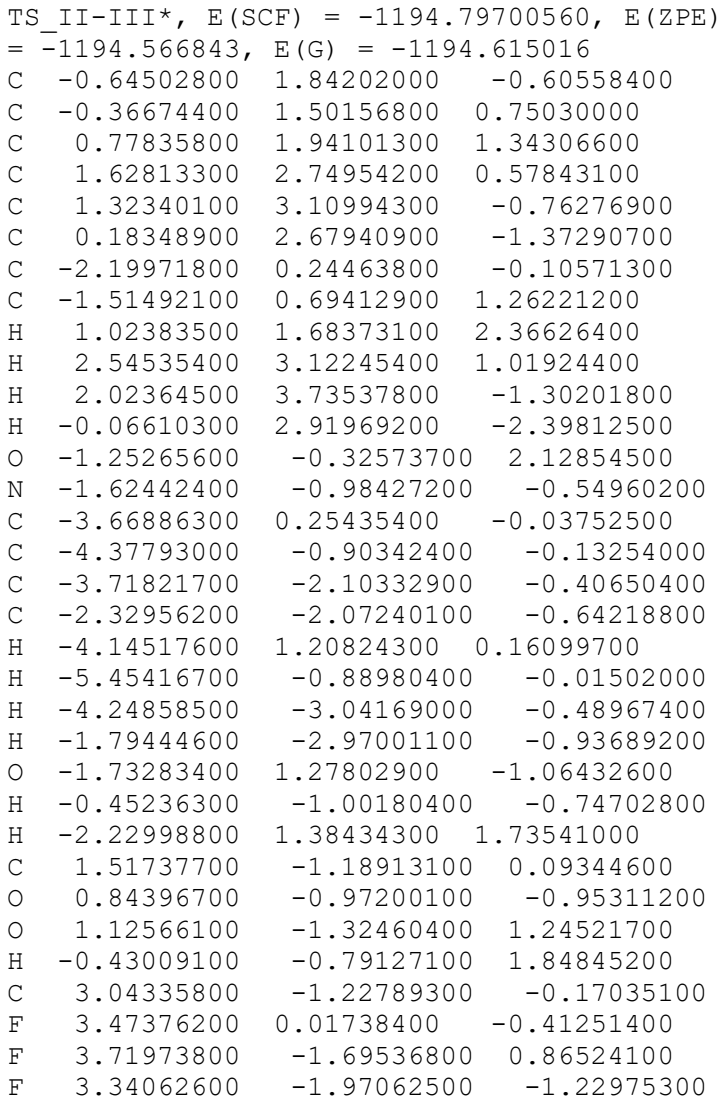

25

TS III-IV, $\mathrm{E}(\mathrm{SCF})=-667.971372752, \mathrm{E}(\mathrm{ZPE})$ $=-667.779713, \mathrm{E}(\mathrm{G})=-667.818395$

$\begin{array}{lrcc}\mathrm{C} & -1.12791500 & -0.64303900 & -0.43656900 \\ \mathrm{C} & -1.31730400 & 0.53120300 & 0.36140800 \\ \mathrm{C} & -2.55610000 & 0.85839900 & 0.81586900 \\ \mathrm{C} & -3.63299400 & 0.02993600 & 0.46073700 \\ \mathrm{C} & -3.45721000 & -1.11167000 & -0.35203500 \\ \mathrm{C} & -2.22528900 & -1.46439900 & -0.81481500 \\ \mathrm{C} & 1.06111600 & 0.26252700 & 0.11592800 \\ \mathrm{C} & -0.02766500 & 1.29318400 & 0.43270700 \\ \mathrm{H} & -2.71900000 & 1.74280500 & 1.42002800 \\ \mathrm{H} & -4.62909700 & 0.27567300 & 0.81005100 \\ \mathrm{H} & -4.32178500 & -1.71461800 & -0.60063700 \\ \mathrm{H} & -2.05559100 & -2.33926900 & -1.42932100 \\ \mathrm{O} & -0.06073400 & 2.31795300 & -0.51437100 \\ \mathrm{~N} & 1.95142600 & 0.69460200 & -0.85194800 \\ \mathrm{C} & 1.52453500 & -0.56720700 & 1.21858300 \\ \mathrm{C} & 2.78501500 & -1.09357200 & 1.16972300 \\ \mathrm{C} & 3.60715200 & -0.76099300 & 0.09855000 \\ \mathrm{C} & 3.13803500 & 0.17430400 & -0.86475100 \\ \mathrm{H} & 0.83770400 & -0.77515300 & 2.03319500 \\ \mathrm{H} & 3.14295500 & -1.74334000 & 1.95866000 \\ \mathrm{H} & 4.60779200 & -1.16333700 & 0.00570800 \\ \mathrm{H} & 3.81219100 & 0.50540600 & -1.65058500 \\ \mathrm{O} & 0.08195900 & -0.89140000 & -0.75672700 \\ \mathrm{H} & 0.71803200 & 2.19339900 & -1.08724000 \\ \mathrm{H} & 0.14876700 & 1.69174200 & 1.44054900\end{array}$

25

TS IV-3a-prot, $\mathrm{E}(\mathrm{SCF})=-667.970297608$, $\mathrm{E}(\overline{\mathrm{Z}} \mathrm{PE})=-667.781818, \mathrm{E}(\mathrm{G})=-667.821049$

$\begin{array}{lrcc}\text { C } & 1.61830400 & -0.84828000 & -0.44756300 \\ \text { C } & 1.20289100 & 0.02765600 & 0.63457700 \\ \text { C } & 1.92756000 & 1.13733800 & 0.94278200 \\ \text { C } & 3.05877100 & 1.46248600 & 0.18076600 \\ \text { C } & 3.46418800 & 0.65451000 & -0.89370400 \\ \text { C } & 2.77498500 & -0.47342400 & -1.22088800 \\ \text { C } & -1.30137100 & 0.15376400 & 0.51449000\end{array}$

C $\quad-0.09292300$

$\mathrm{H} \quad 1.63476600$

$\mathrm{H} \quad 3.63122100$

H $\quad 4.34772000$

$\mathrm{H} \quad 3.07135200$

O -0.21864200

$\mathrm{N}-2.04208100$

C -1.62697700

C -2.78943300

C -3.56060700

C -3.15648700

$\mathrm{H} \quad-0.99809900$

$\mathrm{H}-3.09392300$

$\mathrm{H} \quad-4.46925600$

$\mathrm{H}-3.71561200$

O 0.97840600

$\mathrm{H} \quad-1.21729500$

$\mathrm{H} \quad-0.08781600$

$-0.39108300 \quad 1.27366400$

$1.76729800 \quad 1.77531300$

$2.34760000 \quad 0.43103900$

$0.92601300-1.45876000$

$-1.12535000-2.03260000$

$-1.76432800 \quad 1.24880000$

$\begin{array}{ll}-0.86576000 & 0.10484700\end{array}$

$\begin{array}{lll}1.46363200 & 0.24879000\end{array}$

$1.68980600-0.47226000$

$0.61868700-0.89965900$

$-0.66578100 \quad-0.59183300$

$2.27643700 \quad 0.59223700$

$2.70318300-0.70483700$

$0.77698500-1.46527100$

$-1.54174200 \quad-0.89795900$

$-1.88754400 \quad-0.69213600$

$-1.79668700 \quad 0.64210400$

$-0.03430500 \quad 2.31654100$

35

1d-prot, $\mathrm{E}(\mathrm{SCF})=-899.039661953, \mathrm{E}(\mathrm{ZPE})=$ $-898.763702, E(G)=-898.809741$

C $0.52782900 \quad 0.47246100-0.55798800$

C $\quad 0.72006300 \quad 1.79687600 \quad-0.15901600$

$\begin{array}{llll}\text { C } & -0.40164200 & 2.48675000 & 0.29570800\end{array}$

C $-1.64267700 \quad 1.88864500 \quad 0.35777700$

C $-1.81687300 \quad 0.56576300 \quad-0.05026500$

C $-0.70043600 \quad-0.13662300 \quad-0.51593900$

C $\quad 2.55468000-0.67987700 \quad-0.29950400$

C $\quad 2.00276500 \quad 2.50819800 \quad-0.20855700$

$\mathrm{H} \quad-0.28130300 \quad 3.52346800 \quad 0.59489700$

H $\quad-2.49609600 \quad 2.46375400 \quad 0.69635000$

$\mathrm{H}-0.78370300 \quad-1.16915100 \quad-0.83510400$

$\begin{array}{llll}0 & 3.07190100 & 2.02928000 & -0.50414400\end{array}$

$\mathrm{N} \quad 3.65918000 \quad-1.06109600 \quad-0.95599700$

C $\quad 2.50755700 \quad-0.80027300 \quad 1.07923900$

C $\quad 3.61333800-1.30037100 \quad 1.72926800$

C $\quad 4.75240400 \quad-1.68298200 \quad 1.02171800$

C $4.74946800-1.55431800 \quad-0.33587700$

H $\quad 1.61485800 \quad-0.50516900 \quad 1.61467200$

$\mathrm{H} \quad 3.59115000 \quad-1.40174800 \quad 2.80749900$

$\mathrm{H} \quad 5.62438800 \quad-2.07610400 \quad 1.52422100$

$\mathrm{H} \quad 5.57831400 \quad-1.82214700 \quad-0.97589000$

o $1.58337500-0.26464700-1.09273700$

$\mathrm{H} \quad 3.67074700 \quad-0.94478600 \quad-1.96657700$

$\mathrm{H} \quad \begin{array}{llll}1.91590500 & 3.58119700 & 0.06351700\end{array}$

C $-3.13802200 \quad-0.08274000 \quad-0.00082600$

C $-3.51281600-1.00704200-0.97648100$

C $\quad-4.04203300 \quad 0.21506200 \quad 1.01954400$

C $-4.75682300-1.61100000 \quad-0.93601400$

$\mathrm{H}-2.83435400 \quad-1.23458800 \quad-1.79183100$

C $\quad-5.28278400 \quad-0.39571400 \quad 1.06254100$

$\mathrm{H} \quad-3.76390900 \quad 0.91397200 \quad 1.80108800$

C $\quad-5.64518900 \quad-1.30897700 \quad 0.08450200$

$\mathrm{H} \quad-5.03718900 \quad-2.31636300 \quad-1.70995800$

$\mathrm{H} \quad-5.96909700 \quad-0.16081700 \quad 1.86815300$

$\mathrm{H} \quad-6.61903700 \quad-1.78392700 \quad 0.11702600$

35

1e-prot, $\mathrm{E}(\mathrm{SCF})=-899.038430008, \mathrm{E}(\mathrm{ZPE})=$ $-898.762097, \mathrm{E}(\mathrm{G})=-898.807587$

$\begin{array}{llll}\text { C } & 0.80667000 & 0.05786000 & 0.91107200\end{array}$

C $\quad 0.26272500 \quad-0.97857100 \quad 0.15140600$

C $-1.10000000-0.93744000 \quad-0.12187200$

C $-1.91483100 \quad 0.09693200 \quad 0.32910400$

C $-1.32505100 \quad 1.10463600 \quad 1.09040600$

C $\quad 0.02892600 \quad 1.08730200 \quad 1.38470400$

C $\quad 3.07900300 \quad 0.29739600 \quad 0.38653000$

C $1.03456600 \quad-2.11644100 \quad-0.37633800$

$\mathrm{H}-1.52263400 \quad-1.73667200 \quad-0.72418300$

$\mathrm{H} \quad-1.93703600 \quad 1.91030400 \quad 1.48019200$

H $\quad 0.48018500 \quad 1.86410700 \quad 1.99074000$

O $2.23062800 \quad-2.25872900 \quad-0.30251100$

$\mathrm{N} \quad 4.31170100 \quad-0.07627500 \quad 0.75830400$

$\begin{array}{llll}\text { C } & 2.90188300 & 0.94308500 & -0.82576600\end{array}$

C $4.00753500 \quad 1.17001800 \quad-1.61398400$

C $\quad 5.27810100 \quad 0.76772700-1.20398900$ 


\begin{tabular}{|c|c|c|c|}
\hline $\mathrm{C}$ & 5.40545700 & 0.14172600 & 0.00080000 \\
\hline $\mathrm{H}$ & 1.91152000 & 1.25625700 & -1.12876900 \\
\hline & 3.88368400 & 1.67448700 & -2.56465100 \\
\hline $\mathrm{H}$ & 6.15167700 & 0.94354900 & -1.81499800 \\
\hline & 6.34271900 & -0.20779700 & 0.40960400 \\
\hline & 2.14856500 & 0.03351500 & 1.28630600 \\
\hline $\mathrm{H}$ & 4.41129800 & -0.56506500 & 1.64511800 \\
\hline $\mathrm{H}$ & 0.40066300 & -2.87666100 & -0.87868100 \\
\hline C & -3.35584600 & 0.12411000 & 0.01424100 \\
\hline C & -4.10251900 & -1.05322000 & -0.01187300 \\
\hline C & -3.99913900 & 1.32929400 & -0.26544500 \\
\hline C & -5.45452100 & -1.02508500 & -0.30702800 \\
\hline $\mathrm{H}$ & -3.62508100 & -1.99833600 & 0.22482300 \\
\hline C & -5.35058600 & 1.35588000 & -0.56308300 \\
\hline $\mathrm{H}$ & -3.43082700 & 2.25354700 & -0.27133300 \\
\hline C & -6.08299600 & 0.17905800 & -0.58414500 \\
\hline $\mathrm{H}$ & -6.02255200 & -1.94850200 & -0.31276500 \\
\hline $\mathrm{H}$ & -5.83348300 & 2.30052600 & -0.78656800 \\
\hline $\mathrm{H}$ & -7.14184500 & 0.20029700 & -0.81544700 \\
\hline \multicolumn{4}{|c|}{30} \\
\hline \multicolumn{2}{|c|}{$\begin{array}{l}\text { 1f-prot, } E(\mathrm{SCF})= \\
-837.470192, \mathrm{E}(\mathrm{G})\end{array}$} & \multicolumn{2}{|c|}{$\begin{array}{l}=-837.700379833, \mathrm{E}(\mathrm{ZPE})= \\
=-837.512575\end{array}$} \\
\hline C & -2.35216600 & -0.71839300 & -0.32606900 \\
\hline C & -1.04006100 & -0.99105500 & -0.77629800 \\
\hline C & -0.08873600 & -0.02925900 & -0.68058300 \\
\hline C & -0.35292300 & 1.26258800 & -0.15318300 \\
\hline C & -1.62911500 & 1.52766000 & 0.28068500 \\
\hline C & -2.64035800 & 0.55643100 & 0.21629500 \\
\hline $\mathrm{H}$ & -0.79579100 & -1.95847500 & -1.20019000 \\
\hline $\mathrm{H}$ & -1.85999200 & 2.51140900 & 0.68156400 \\
\hline C & 0.63806200 & 2.34424600 & -0.05870900 \\
\hline $\mathrm{H}$ & 0.20169000 & 3.29555600 & 0.31014200 \\
\hline O & 1.81055600 & 2.26420400 & -0.33444800 \\
\hline O & 1.17289500 & -0.31404100 & -1.19665300 \\
\hline C & 2.19017700 & -0.46641600 & -0.36491100 \\
\hline C & 2.12072600 & -0.74875300 & 0.98838800 \\
\hline $\mathrm{N}$ & 3.38813100 & -0.37632400 & -0.95837700 \\
\hline C & 3.29946000 & -0.91035000 & 1.68276000 \\
\hline $\mathrm{H}$ & 1.15742100 & -0.83767000 & 1.47253200 \\
\hline C & 4.55029700 & -0.53486700 & -0.29562300 \\
\hline C & 4.53249200 & -0.80241200 & 1.04191600 \\
\hline $\mathrm{H}$ & 3.26211400 & -1.13069000 & 2.74283500 \\
\hline $\mathrm{H}$ & 5.45086600 & -0.43296500 & -0.88458300 \\
\hline $\mathrm{H}$ & 5.46175300 & -0.92897300 & 1.57837700 \\
\hline $\mathrm{C}$ & -4.63191800 & -1.29444700 & 0.08979500 \\
\hline $\mathrm{H}$ & -5.45628200 & -2.00016600 & 0.05944700 \\
\hline $\mathrm{C}$ & -3.39913900 & -1.65436200 & -0.37819000 \\
\hline $\mathrm{H}$ & -3.22845900 & -2.64355300 & -0.78686600 \\
\hline $\mathrm{H}$ & 3.40653100 & -0.14091500 & -1.94783900 \\
\hline $\mathrm{C}$ & -3.95709900 & 0.80339800 & 0.66461200 \\
\hline $\mathrm{H}$ & -4.19219200 & 1.78191800 & 1.08102400 \\
\hline $\mathrm{N}$ & -4.91862300 & -0.07808100 & 0.60938400 \\
\hline
\end{tabular}

